\title{
Copper-Catalyzed Asymmetric Borylacylation of Styrene and Indene Derivatives
}

\author{
Zhen Yang ${ }^{\dagger}$, Peiyuan $\mathrm{Li}^{\dagger}$, Hongjian Lu, ${ }^{\dagger} * *$ Guigen $\mathrm{Li}^{\dagger}{ }^{\dagger+}, * *$ \\ ${ }^{\dagger}$ Institute of Chemistry and BioMedical Sciences, Jiangsu Key Laboratory of Advanced Organic \\ Materials, School of Chemistry and Chemical Engineering, Nanjing University, Nanjing, 210093, \\ China \\ ${ }^{*}$ Department of Chemistry and Biochemistry, Texas Tech University, Lubbock, TX 79409-1061, USA \\ guigenli@nju.edu.cn
}

Optimization of Copper-Catalyzed Borylacylation.................................................................

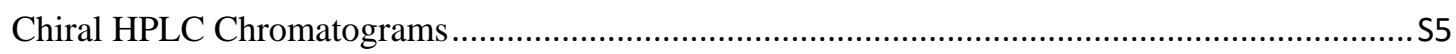

Absolute Stereochemistry Determination ……………......................................................

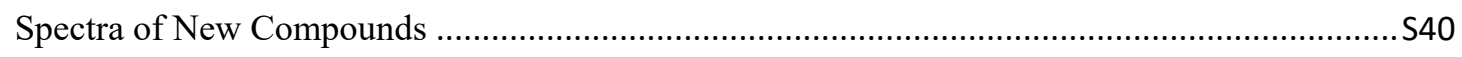




\section{Optimization of Copper-Catalyzed Borylacylation}

Table S1. Ligand screening ${ }^{a}$

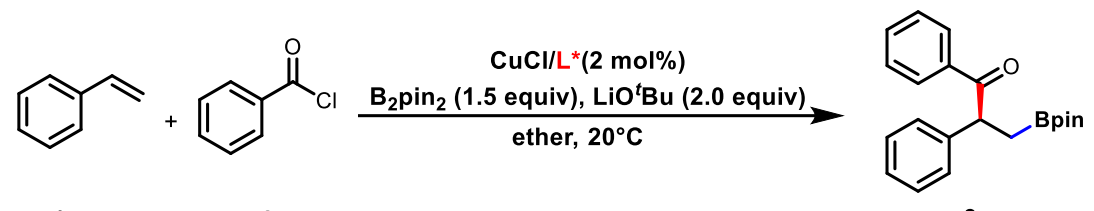

$1 a$

2a

3a<smiles>Cc1cc(C)c(N2CC[C@H](c3ccccc3)P2CCP2[C@H](Pc3ccccc3)CC[C@H]2c2ccccc2)c(C)c1</smiles>

L1: $88 \%$<smiles>Cc1cc(C)c(N2CN(c3ccccc3[O+])[C@H](c3ccccc3)[C@H]2c2ccccc2)c(C)c1</smiles>

L4: n.d.<smiles>Cc1ccccc1N1C(C)C(c2ccccc2)[C@@H](c2ccccc2)N1c1ccccc1</smiles>

L7: $36 \%, 42 \%$ ee

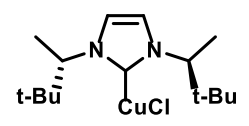

L10: $45 \%,-70 \%$ ee
L2: n.d.<smiles>Cc1cc(C)c(N2C(C(Cl)(Cl)c3ccccc3)[C@@H](c3ccccc3)[C@H](c3ccccc3)N2c2c(C)cc(C)cc2C)c(C)c1</smiles>

L5: $78 \%, 14 \%$ ee<smiles>CCC1N(c2c(C)cc(Cl)cc2C)[C@H](c2ccccc2)[C@@H](c2ccccc2)N1c1c(C)cc(Cl)cc1C</smiles>

L8: $12 \%,--$<smiles>Cc1cc(C)c(N2c3ccccc3C3=N[C@H](c4ccccc4)[C@H](c4ccccc4)N3C2C(Cl)Cl)c(C)c1</smiles>

L11: $72 \%, 87 \%$ ee<smiles>CC(C)(C1=N[C@@H](Br)CO1)C1=N[C@H](Br)CO1</smiles>

L3: n.d.
L6: $48 \%, 8 \%$ ee<smiles>CC(c1ccccc1)N1C(C(Cl)(Cl)c2ccccc2)[C@H](c2ccccc2)C([C@H](C)c2ccccc2)N1Cc1ccccc1</smiles>

L9: $81 \%, 32 \%$ ee<smiles>Cc1cccc(C)c1N1c2ccccc2C2=N[C@@H](c3ccccc3)[C@H](c3ccccc3)N2C1C(Cl)(Cl)c1ccccc1</smiles>

L12:54\%, $70 \%$ ee

${ }^{a}$ Reactions were performed with $1 \mathrm{a}(0.2 \mathrm{mmol}), 2 \mathrm{a}\left(0.3 \mathrm{mmol}, 1.5\right.$ equiv), LiO ${ }^{t} \mathrm{Bu}(0.4 \mathrm{mmol}, 2.0$ equiv),

Bis(pinacolato)diboron $(0.3 \mathrm{mmol}, 1.5$ equiv), cat. $(0.004 \mathrm{mmol}, 2 \mathrm{~mol} \%)$, ligand $(0.004 \mathrm{mmol}, 2 \mathrm{~mol} \%)$ in $2 \mathrm{~mL}$ ether under $\operatorname{Ar}$ for $0.5 \mathrm{~h}$. 
Table S2. Different solvents ${ }^{a}$

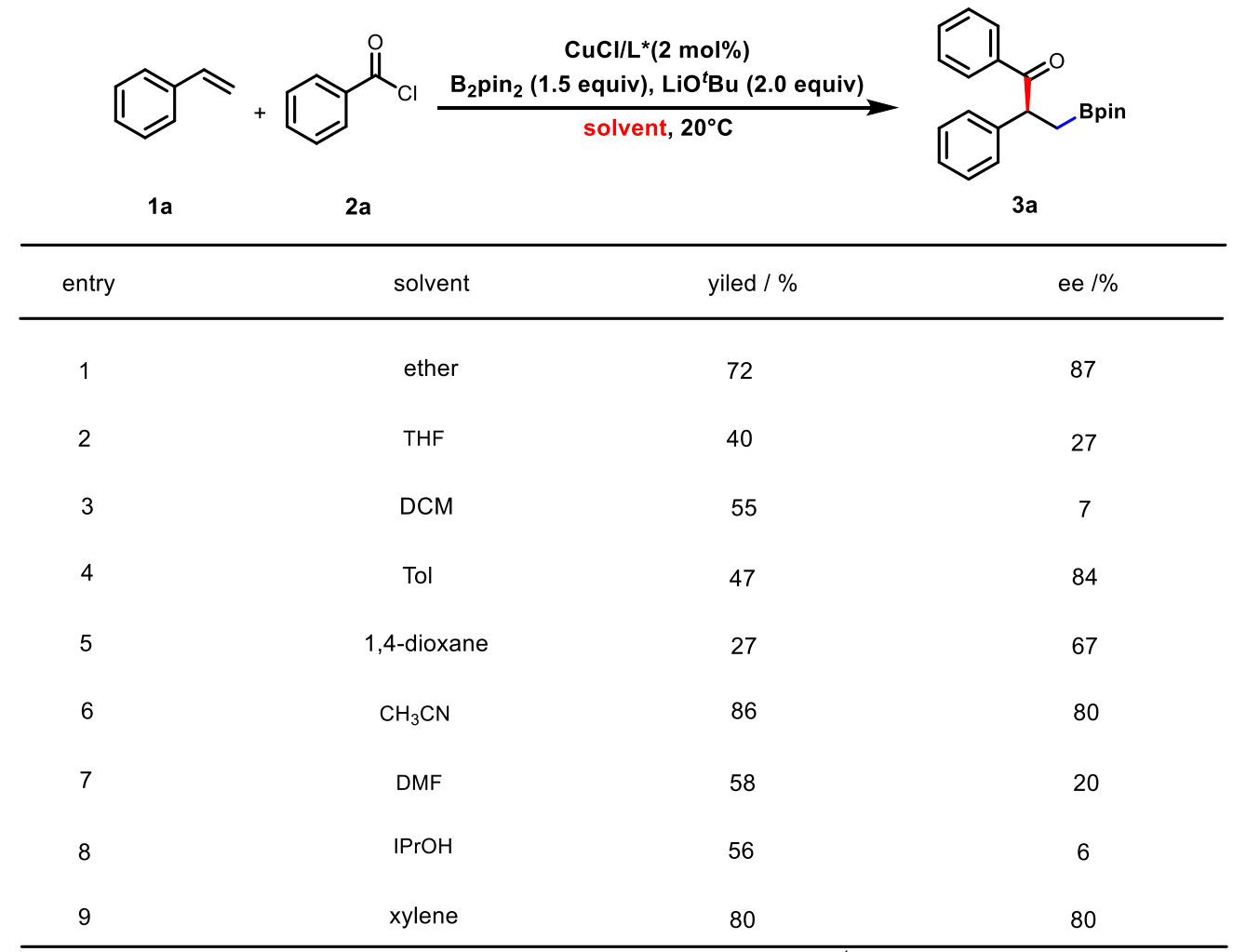

${ }^{a}$ Reactions were performed with 1a $(0.2 \mathrm{mmol}), 2 \mathrm{a}\left(0.3 \mathrm{mmol}, 1.5\right.$ equiv), $\mathrm{LiO}{ }^{\mathrm{t}} \mathrm{Bu}(0.4 \mathrm{mmol}, 2.0$ equiv), Bis(pinacolato)diboron ( $0.3 \mathrm{mmol}, 1.5$ equiv), cat. $(0.004 \mathrm{mmol}, 2 \mathrm{~mol} \%$ ), ligand $(0.004 \mathrm{mmol}, 2 \mathrm{~mol} \%)$ in $2 \mathrm{~mL}$ solvent under $\mathrm{Ar}$ for $0.5 \mathrm{~h}$.

Table S3. Different bases and the amount of base ${ }^{a}$

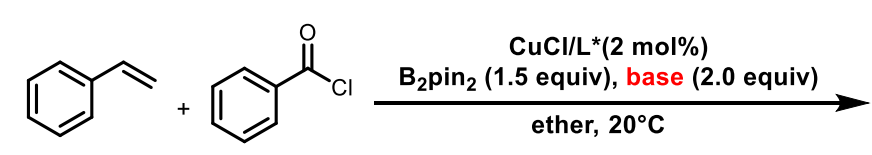

1a

2a

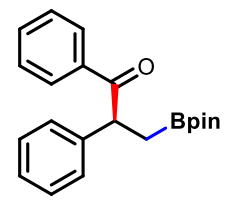

3a

\begin{tabular}{cccc}
\hline entry & base & yiled/ \% & ee /\% \\
\hline 1 & $\mathrm{~K}_{2} \mathrm{CO}_{3}$ & n.d. & - \\
2 & $\mathrm{LiCO}_{3}$ & n.d. & - \\
3 & LiOMe & n.d. & - \\
4 & NaOtBu & 70 & 80 \\
5 & KOtBu & 55 & 72 \\
6 & 1.5 eq LiOtBu & 76 & 90 \\
7 & 1.0 eq LiOtBu & 66 & 81 \\
\hline
\end{tabular}

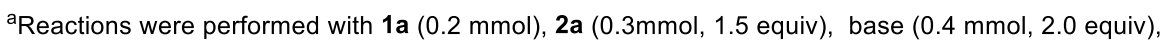
Bis(pinacolato)diboron ( $0.3 \mathrm{mmol}, 1.5$ equiv), cat. $(0.004 \mathrm{mmol}, 2 \mathrm{~mol} \%)$, ligand $(0.004 \mathrm{mmol}, 2 \mathrm{~mol} \%)$ in $2 \mathrm{~mL}$ ether under $\mathrm{Ar}$ for $0.5 \mathrm{~h}$. 
Table S4. Different temperature, reaction time and the amount of catalyst ${ }^{a}$

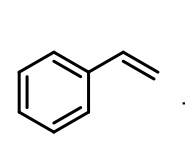

$1 a$<smiles>O=C(Cl)c1ccccc1</smiles>

$2 a$

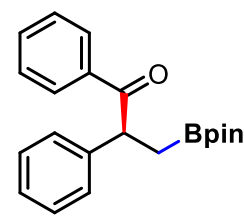

$3 a$

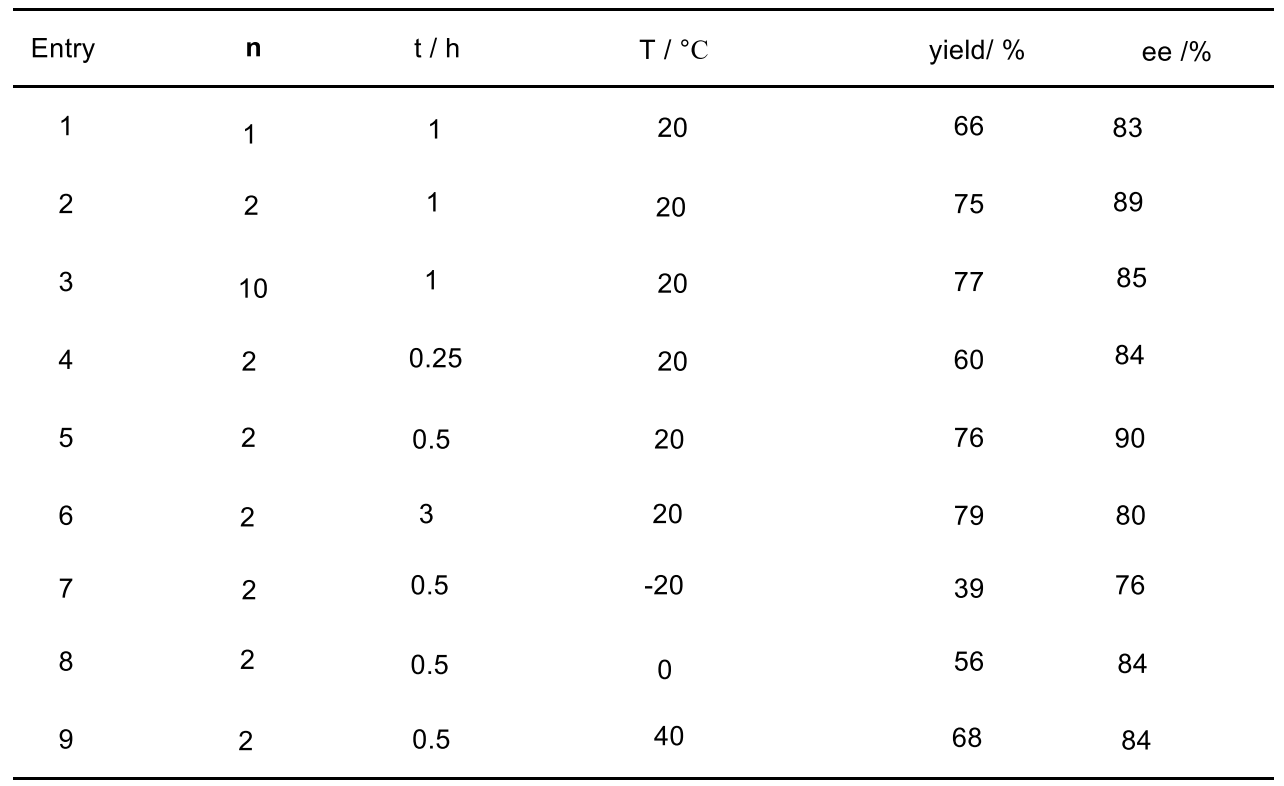

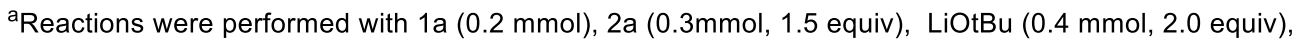
Bis(pinacolato)diboron ( $0.3 \mathrm{mmol}, 1.5$ equiv), cat. ( $\mathrm{n} \mathrm{mol} \%$ ), ligand ( $\mathrm{mol} \%$ ) in $2 \mathrm{~mL}$ ether under $\mathrm{Ar}$

Figure S1. Failed substrates
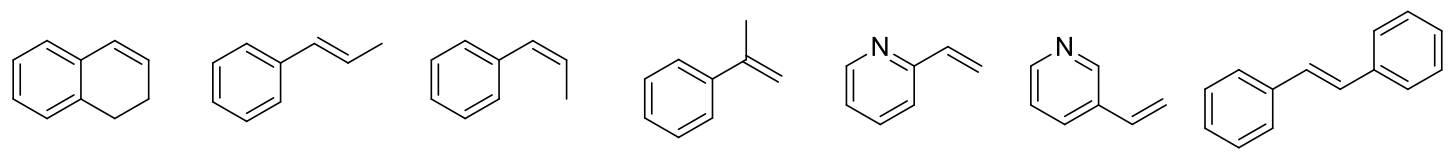<smiles>CC(=O)c1ccccc1</smiles><smiles>O=C(Oc1ccc(Cl)cc1)c1ccccc1</smiles><smiles>O=C(OC(=O)c1ccccc1)c1ccccc1</smiles>

SH 


\section{Chiral HPLC Chromatograms}

Note: For each entry, the top HPLC trace is a racemic sample that was prepared using standard conditions, but employing SIMesCuCl as the ligand.

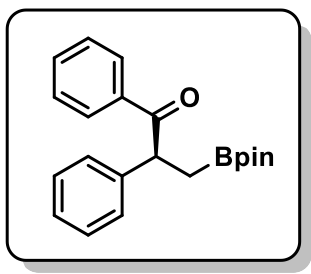

(S)-1,2-diphenyl-3-(4,4,5,5-tetramethyl-1,3,2-dioxaborolan-2-yl)propan-1one (3a): $[\alpha]_{\mathrm{D}}^{24}=+91.9,\left(\mathrm{c}=0.2, \mathrm{CH}_{2} \mathrm{Cl}_{2}\right)$. HPLC analysis (OD-H,

Hexane/IPA $=99 / 1,1 \mathrm{~mL} / \mathrm{min}, 254 \mathrm{~nm}$ ) indicated $90 \%$ ee: $\mathrm{t}_{\mathrm{R}}($ minor $)=7.8$ $\min , t_{R}($ major $)=11.2 \mathrm{~min}$. After the reaction is amplified, the ee value was $88 \%$.

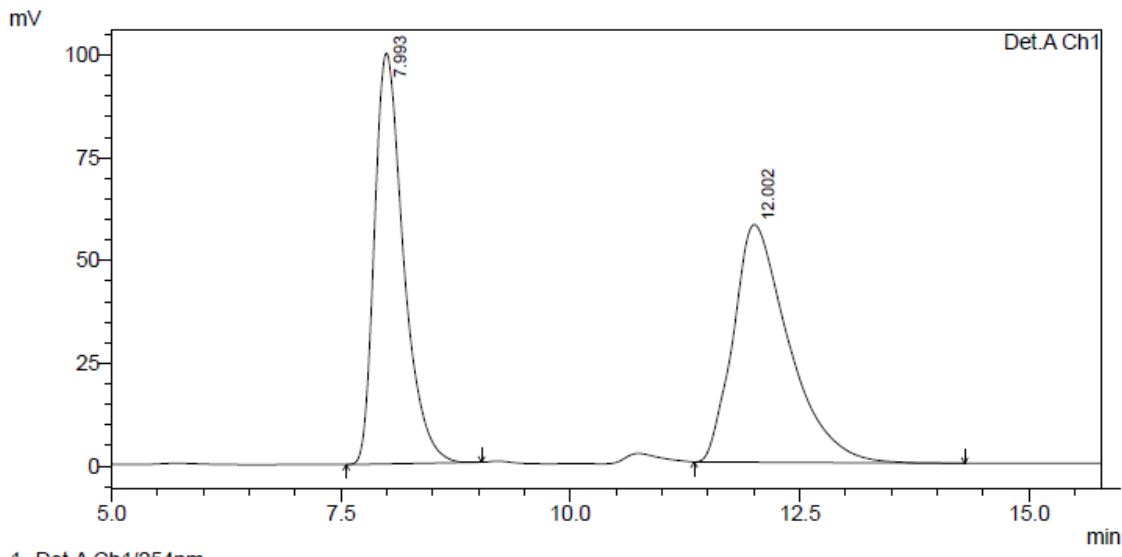

1 Det.A Ch $1 / 254 \mathrm{~nm}$

PeakTable

Detector A Ch1 $254 \mathrm{~nm}$
\begin{tabular}{|r|r|r|r|r|r|}
\hline Peak\# & Ret. Time & \multicolumn{1}{|c|}{ Area } & Height & Area $\%$ & Height $\%$ \\
\hline 1 & 7.993 & 2201080 & 99953 & 47.433 & 63.343 \\
\hline 2 & 12.002 & 2439270 & 57844 & 52.567 & 36.657 \\
\hline Total & & 4640350 & 157797 & 100.000 & 100.000 \\
\hline
\end{tabular}

$\mathrm{mV}$

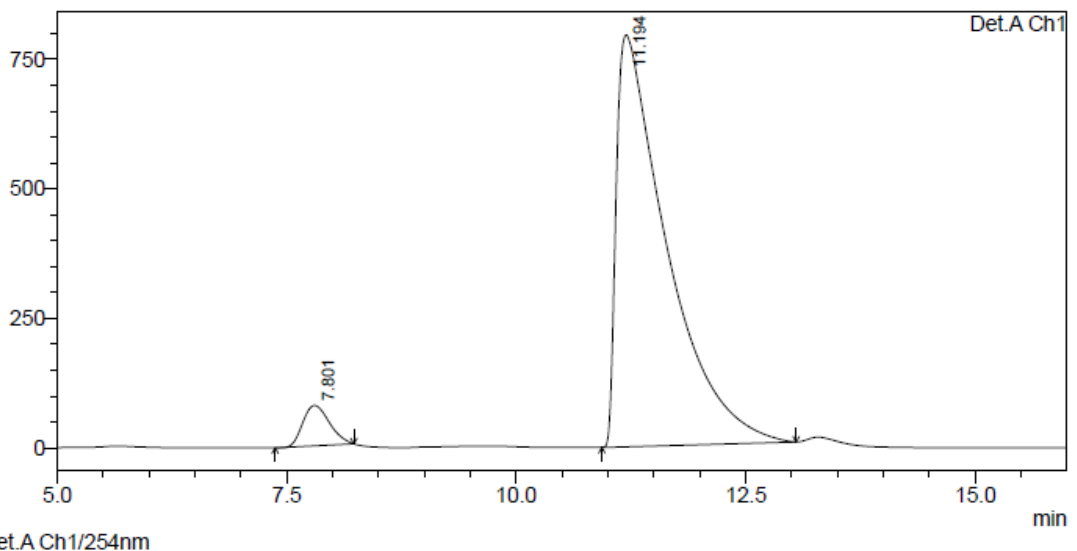

1 Det.A Ch1/254nm

PeakTable

Detector A Ch1 $254 \mathrm{~nm}$
\begin{tabular}{|r|r|r|r|r|r|}
\hline Peakस & Ret. Time & Area & Height & Area \% & Height \% \\
\hline 1 & 7.801 & 1573411 & 77919 & 4.936 & 8.931 \\
\hline 2 & 11.194 & 30301770 & 794574 & 95.064 & 91.069 \\
\hline Total & & 31875181 & 872493 & 100.000 & 100.000 \\
\hline
\end{tabular}




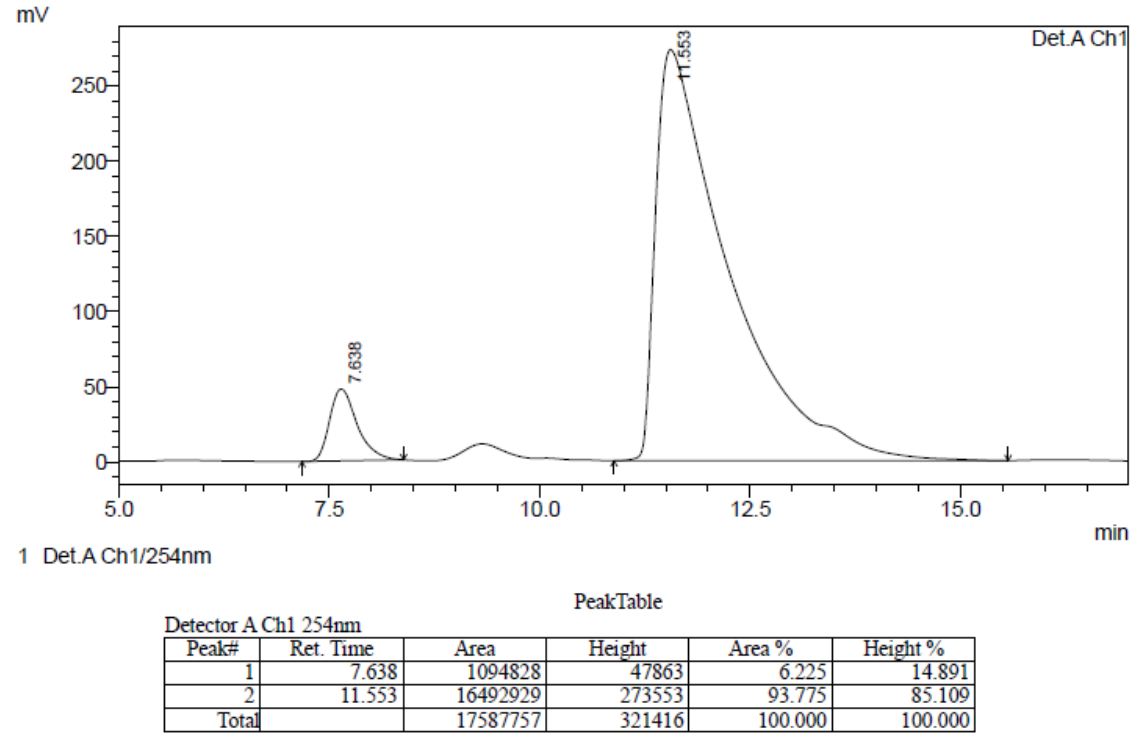




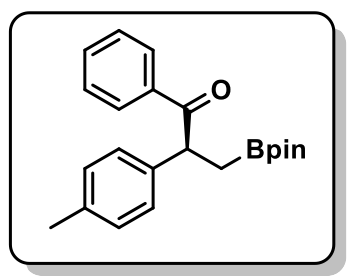

(S)-1-phenyl-3-(4,4,5,5-tetramethyl-1,3,2-dioxaborolan-2-yl)-2-(p-tolyl)

propan-1-one (3b): $[\alpha]_{\mathrm{D}}^{24}=+25.1,\left(\mathrm{c}=0.2, \mathrm{CH}_{2} \mathrm{Cl}_{2}\right)$. HPLC analysis (IF, Hexane $/ \mathrm{IPA}=99 / 1,1 \mathrm{~mL} / \mathrm{min}, 254 \mathrm{~nm})$ indicated $96 \%$ ee: $\mathrm{t}_{\mathrm{R}}($ major $)=$ $12.9 \mathrm{~min}, \mathrm{t}_{\mathrm{R}}($ minor $)=20.9 \mathrm{~min}$.

$\mathrm{mV}$

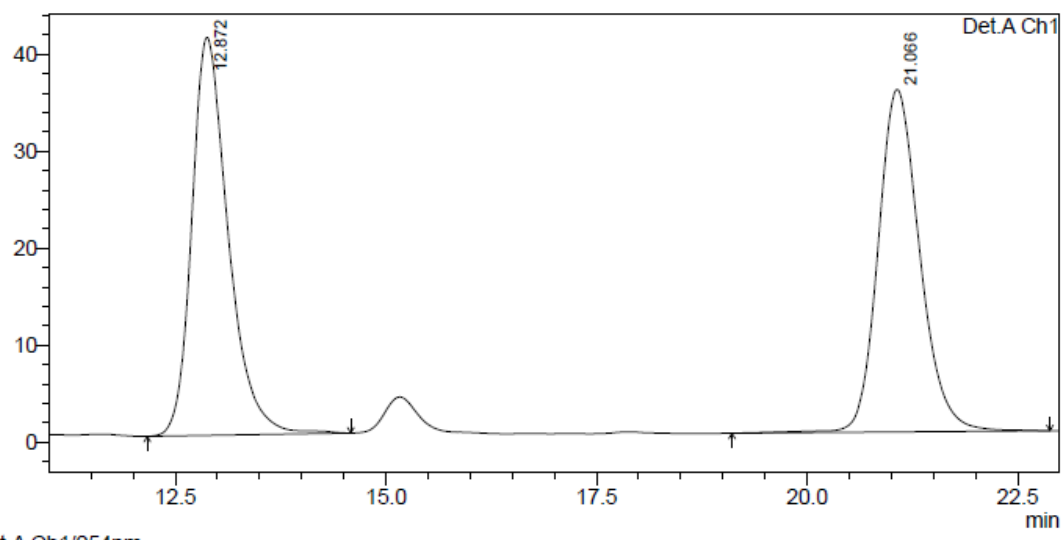

1 Det.A Ch1/254nm

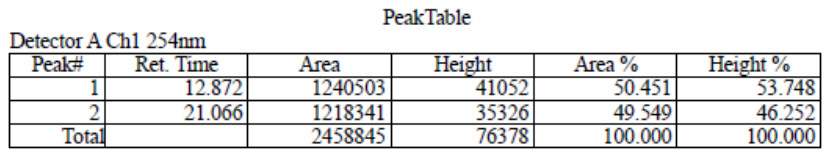

$\mathrm{mV}$

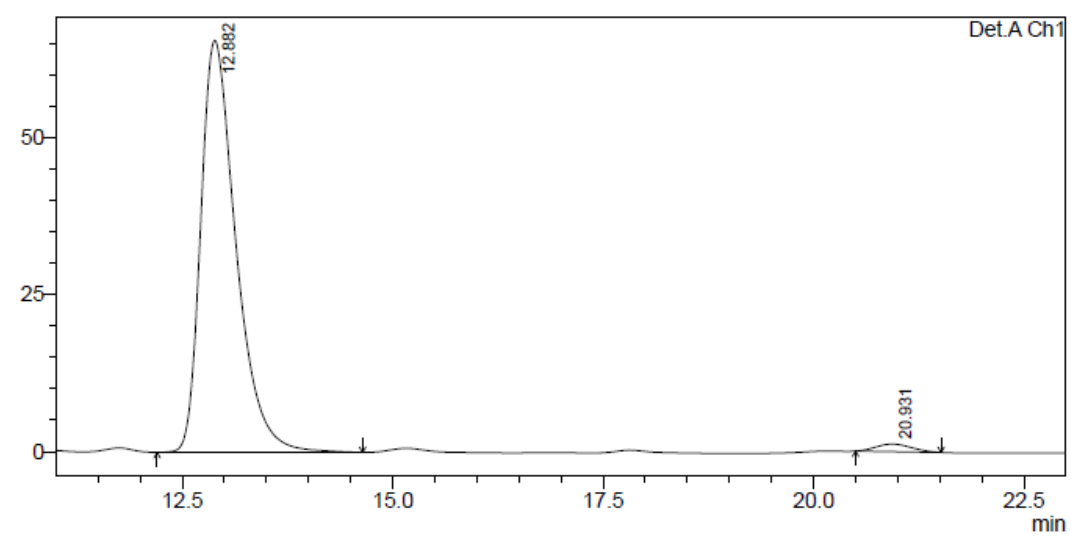

1 Det.A Ch1/254nm

\begin{tabular}{|c|c|c|c|c|c|}
\hline \multicolumn{6}{|c|}{ Detector A Ch1 $254 \mathrm{~nm}$} \\
\hline Peak\# & Ret. Time & Area & Height & Area \% & Height \% \\
\hline 1 & 12.882 & 1914960 & 65703 & 98.181 & 98.192 \\
\hline 2 & 20.931 & 35474 & 1210 & 1.819 & 1.808 \\
\hline Tota & & 1950433 & 66913 & 100.000 & 100.000 \\
\hline
\end{tabular}




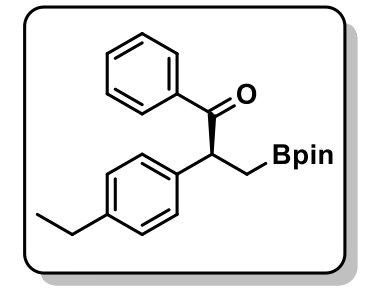

(S)-2-(4-ethylphenyl)-1-phenyl-3-(4,4,5,5-tetramethyl-1,3,2-dioxaborola

n-2-yl)propan-1-one $(3 \mathrm{c}):[\alpha]_{\mathrm{D}}{ }^{24}=+21.3,\left(\mathrm{c}=0.2, \mathrm{CH}_{2} \mathrm{Cl}_{2}\right)$. HPLC analysis (IF, Hexane/IPA = 99/1, $1 \mathrm{~mL} / \mathrm{min}, 254 \mathrm{~nm}$ ) indicated $97 \%$ ee: $\mathrm{t}_{\mathrm{R}}$ $($ major $)=11.8 \min , \mathrm{t}_{\mathrm{R}}($ minor $)=15.6 \mathrm{~min}$

$\mathrm{mV}$

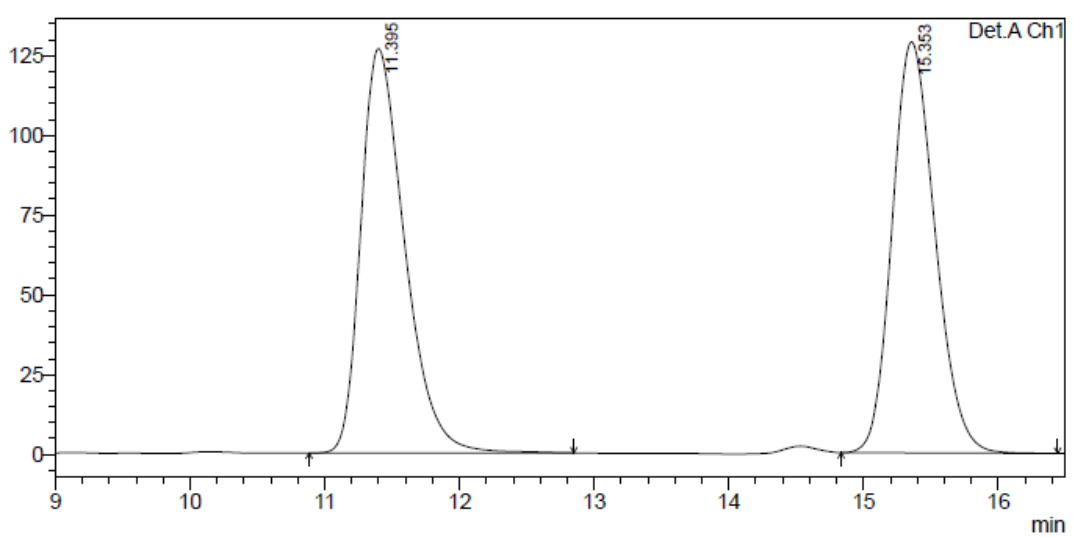

1 Det.A Ch $1 / 254 \mathrm{~nm}$

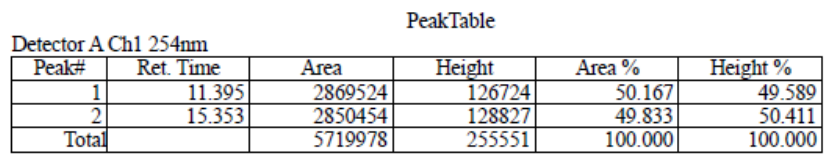

$\mathrm{mV}$

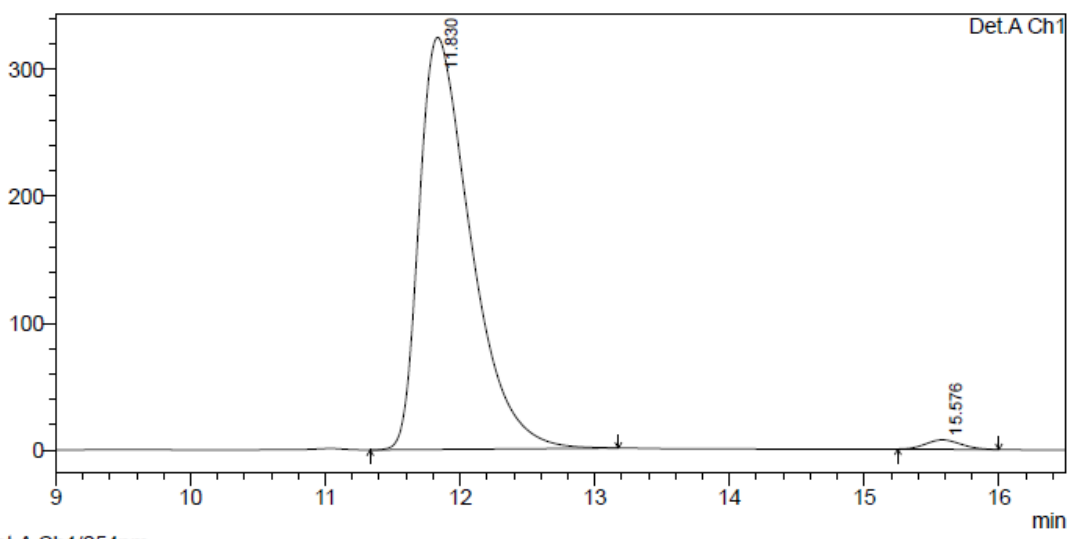

Det A Ch1/254nm

PeakTable

\begin{tabular}{|c|c|c|c|c|c|}
\hline Peak\# & Ret. Time & Area & Height & Area $\%$ & Height \% \\
\hline 1 & 11.830 & 8633346 & 324820 & 98.462 & 97.762 \\
\hline 2 & 15.576 & 134846 & 7436 & 1.538 & 2.238 \\
\hline Total & & 8768193 & 332256 & 100.000 & 100.000 \\
\hline
\end{tabular}




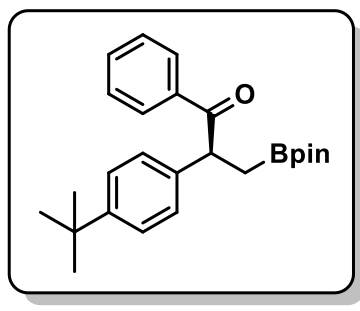

(S)-2-(4-(tert-butyl)phenyl)-1-phenyl-3-(4,4,5,5-tetramethyl-1,3,2-diox

aborolan-2-yl)propan-1-one (3d): $[\alpha]_{\mathrm{D}}^{24}=+120.6,\left(\mathrm{c}=0.2, \mathrm{CH}_{2} \mathrm{Cl}_{2}\right)$.

HPLC analysis (IF, Hexane/IPA = 99/1, $1 \mathrm{~mL} / \mathrm{min}, 254 \mathrm{~nm}$ ) indicated 99\% ee: $t_{R}($ major $)=20.4 \mathrm{~min}, \mathrm{t}_{\mathrm{R}}($ minor $)=27.2 \mathrm{~min}$.

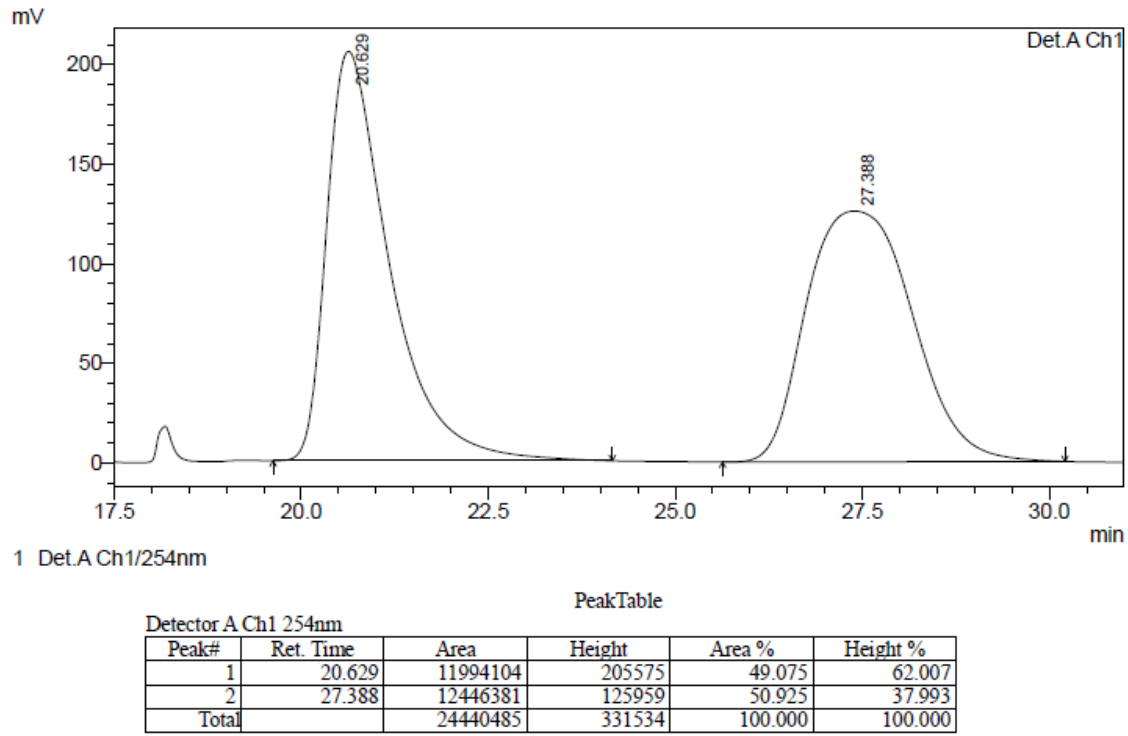

$\mathrm{mV}$

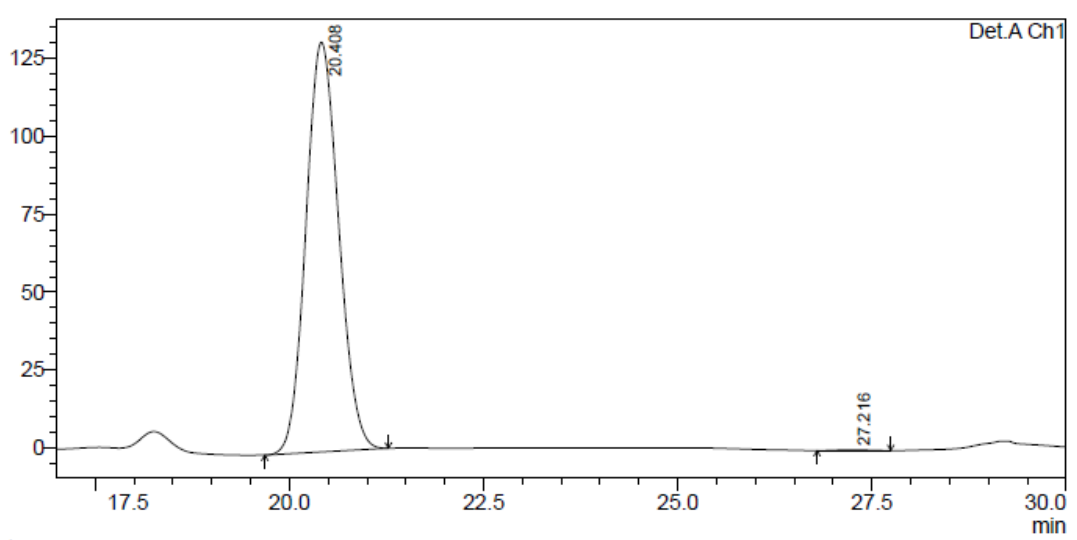

1 Det.A Ch $1 / 254 \mathrm{~nm}$

Detector A Ch1 $254 \mathrm{~nm}$
\begin{tabular}{|r|r|r|r|r|r|}
\hline Peak\# & Ret. Time & \multicolumn{1}{c|}{ Area } & Height & Area $\%$ & \multicolumn{1}{c|}{ Height $\%$} \\
\hline 1 & 20.408 & 3856703 & 131383 & 99.847 & 99.835 \\
\hline 2 & 27.216 & 5895 & 218 & 0.153 & 0.165 \\
\hline Total & & 3862599 & 131600 & 100.000 & 100.000 \\
\hline
\end{tabular}




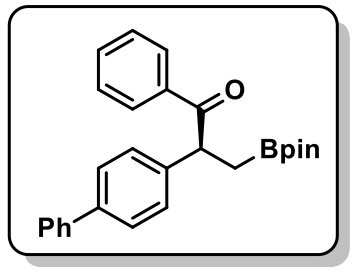

$\mathrm{mV}$

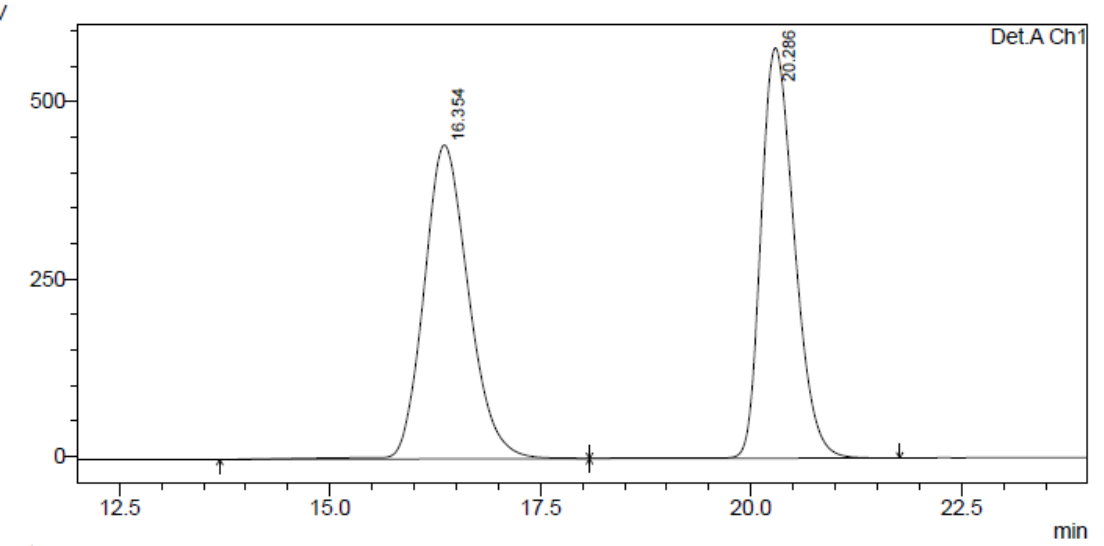

1 Det.A Ch1/254nm

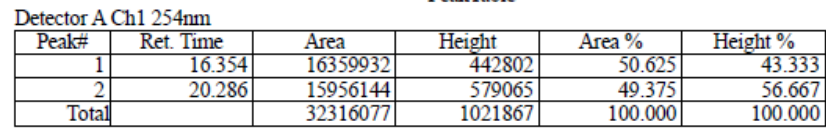

$\mathrm{mV}$

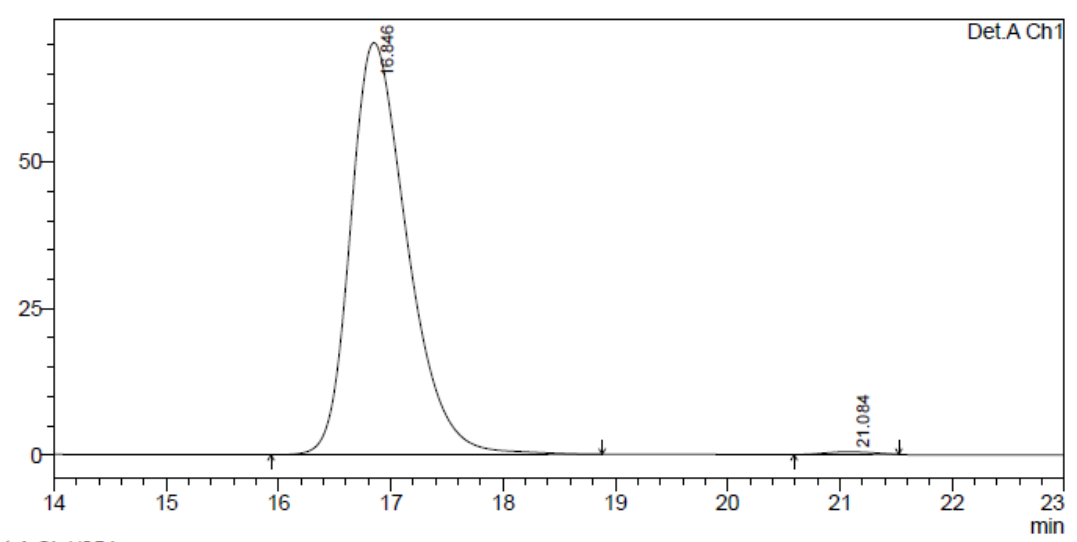

1 Det.A Ch1/254nm

PeakTable

Detector A Ch1 $254 \mathrm{~nm}$
\begin{tabular}{|r|r|r|r|r|r|}
\hline Peak\# & Ret. Time & \multicolumn{1}{|c|}{ Area } & Height & Area $\%$ & \multicolumn{1}{c|}{ Height $\%$} \\
\hline 1 & 16.846 & 2467530 & 70197 & 99.455 & 99.334 \\
\hline 2 & 21.084 & 13512 & 471 & 0.545 & 0.666 \\
\hline Total & & 2481043 & 70668 & 100.000 & 100.000 \\
\hline
\end{tabular}




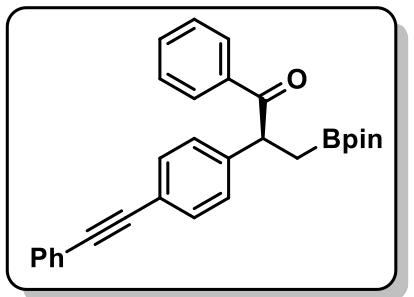

(S)-1-phenyl-2-(4-(phenylethynyl)phenyl)-3-(4,4,5,5-tetramethyl-1,

3,2-dioxaborolan-2-yl)propan-1-one (3f): $[\alpha]_{\mathrm{D}}{ }^{24}=+81.2$., $(\mathrm{c}=0.2$, $\mathrm{CH}_{2} \mathrm{Cl}_{2}$ ). HPLC analysis (IA, Hexane/IPA $=99 / 1,1 \mathrm{~mL} / \mathrm{min}, 254 \mathrm{~nm}$ ) indicated $92 \%$ ee: $t_{R}($ major $)=7.8$ min, $t_{R}($ minor $)=9.4 \mathrm{~min}$.

$\mathrm{mV}$

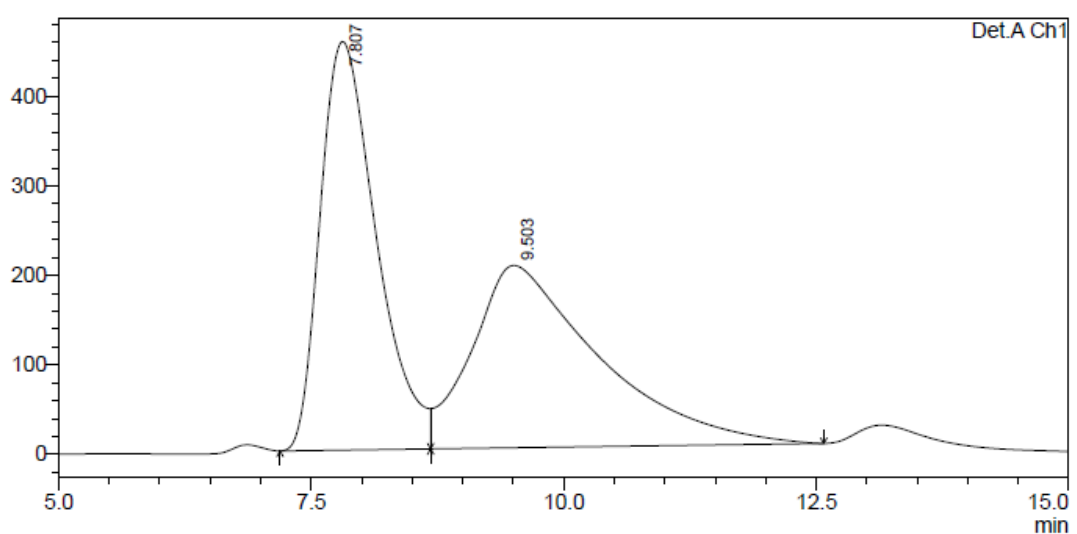

1 Det.A Ch $1 / 254 \mathrm{~nm}$

PeakTable

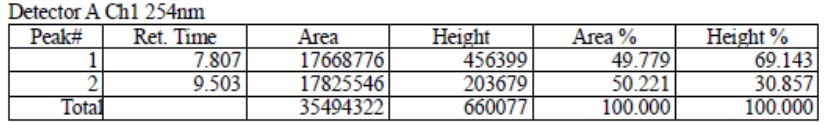

$\mathrm{mV}$

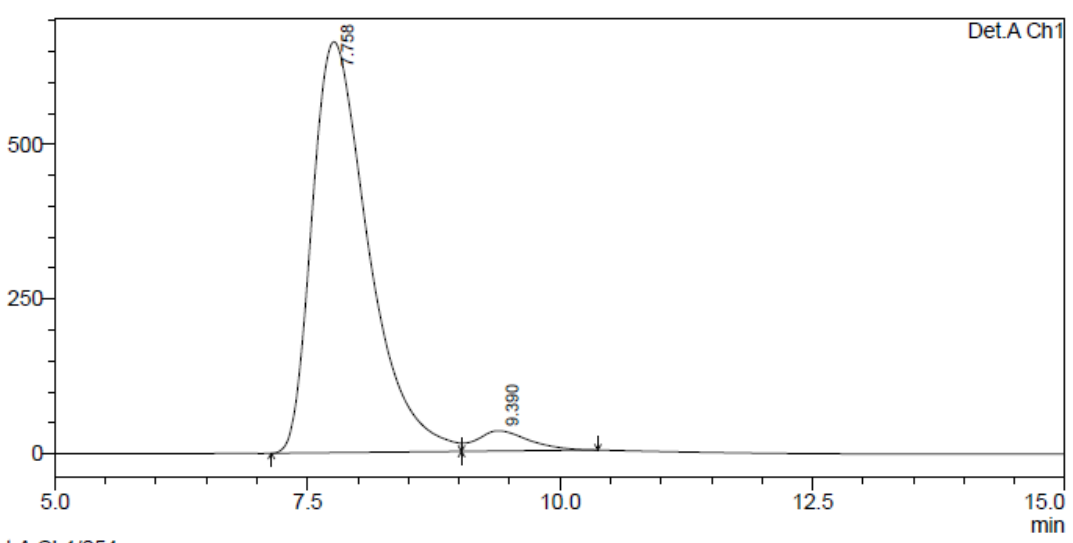

1 Det.A Ch1/254nm

\begin{tabular}{|c|c|c|c|c|c|}
\hline \multicolumn{6}{|c|}{ PeakTable } \\
\hline Peak\# & Ret. Time & Area & Height & Area \% & Height $\%$ \\
\hline & 7.758 & 25773213 & 664103 & 95.635 & 95.312 \\
\hline & 9.390 & 1176338 & 32667 & 4.365 & 4.688 \\
\hline Tota & & 26949551 & 696770 & 100.000 & 100.000 \\
\hline
\end{tabular}




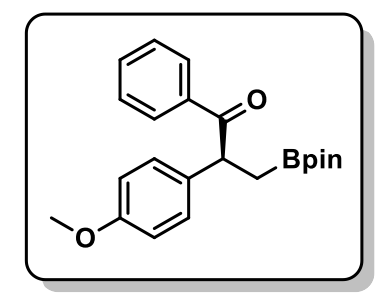

(S)-2-(4-methoxyphenyl)-1-phenyl-3-(4,4,5,5-tetramethyl-1,3,2-dioxab

orolan-2-yl)propan-1-one (3g): $[\alpha]_{\mathrm{D}}{ }^{24}=+143.3$, $\left(\mathrm{c}=0.2, \mathrm{CH}_{2} \mathrm{Cl}_{2}\right)$.

HPLC analysis (OD-H, Hexane/IPA = 99/1, $1 \mathrm{~mL} / \mathrm{min}, 254 \mathrm{~nm})$ indicated $95 \%$ ee: $\mathrm{t}_{\mathrm{R}}($ minor $)=11.3 \mathrm{~min}, \mathrm{t}_{\mathrm{R}}($ major $)=14.3 \mathrm{~min}$.

$\mathrm{mV}$

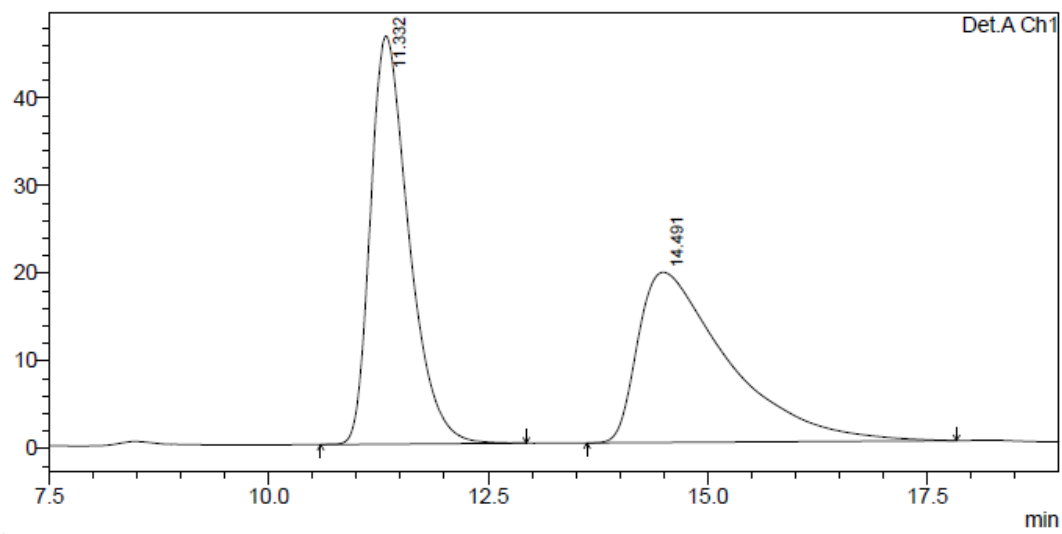

1 Det.A Ch1/254nm

PeakTable
\begin{tabular}{|r|r|r|r|r|r|}
\hline \multicolumn{1}{|c|}{ Detector A Ch1 $254 \mathrm{~nm}$} \\
\hline Peak\# & Ret. Time & \multicolumn{1}{|c|}{ Area } & Height & Area \% & \multicolumn{1}{c|}{ Height \% } \\
\hline 1 & 11.332 & 1421132 & 46559 & 50.608 & 70.567 \\
\hline 2 & 14.491 & 1386959 & 19420 & 49.392 & 29.433 \\
\hline Total & & 2808091 & 65979 & 100.000 & 100.000 \\
\hline
\end{tabular}

$\mathrm{mV}$

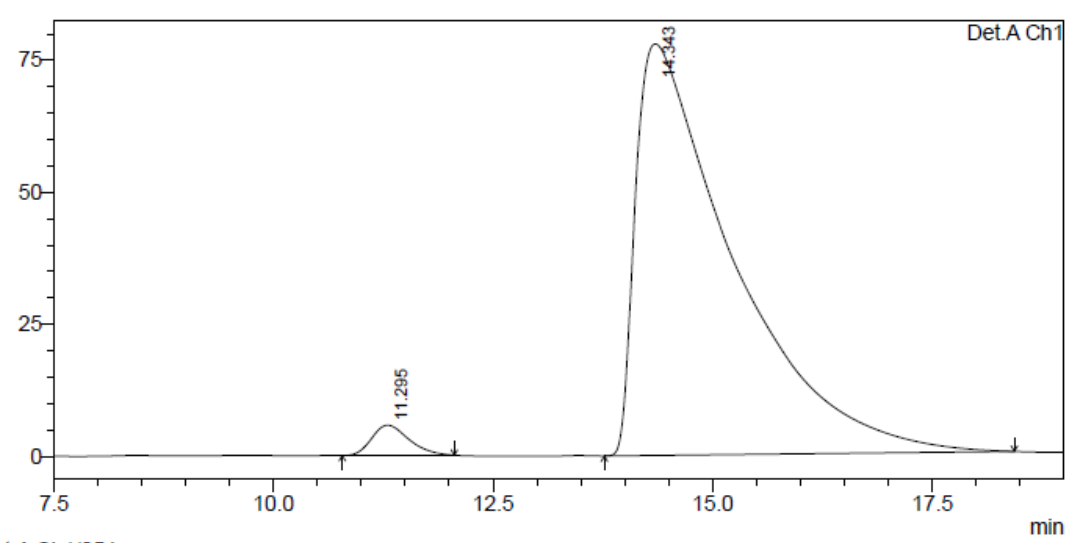

1 Det.A Ch $1 / 254 \mathrm{~nm}$

Detector A Ch1 $254 \mathrm{~nm}$
\begin{tabular}{|r|r|r|r|r|r|}
\hline \multicolumn{1}{|c|}{ Peak\# } & Ret. Time & \multicolumn{1}{c|}{ Area } & \multicolumn{1}{c|}{ Height } & Area $\%$ & \multicolumn{1}{c|}{ Height \% } \\
\hline 1 & 11.295 & 168003 & 5705 & 2.718 & 6.824 \\
\hline 2 & 14.343 & 6012832 & 77897 & 97.282 & 93.176 \\
\hline Tota| & & 6180836 & 83603 & 100.000 & 100.000 \\
\hline
\end{tabular}




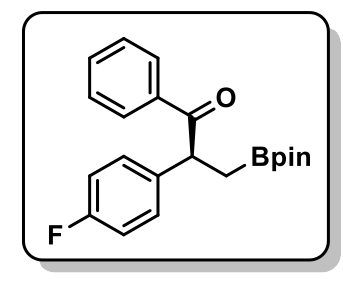

(S)-2-(4-fluorophenyl)-1-phenyl-3-(4,4,5,5-tetramethyl-1,3,2-dioxaborola

n-2-yl)propan-1-one (3h): $[\alpha]_{\mathrm{D}}{ }^{24}=+6.5,\left(\mathrm{c}=0.2, \mathrm{CH}_{2} \mathrm{Cl}_{2}\right)$. HPLC analysis $(\mathrm{IF}$, Hexane $/ \mathrm{IPA}=99 / 1,1 \mathrm{~mL} / \mathrm{min}, 254 \mathrm{~nm})$ indicated $96 \%$ ee: $\mathrm{t}_{\mathrm{R}}($ major $)=$ $11.6 \mathrm{~min}, \mathrm{t}_{\mathrm{R}}($ minor $)=16.2 \mathrm{~min}$.

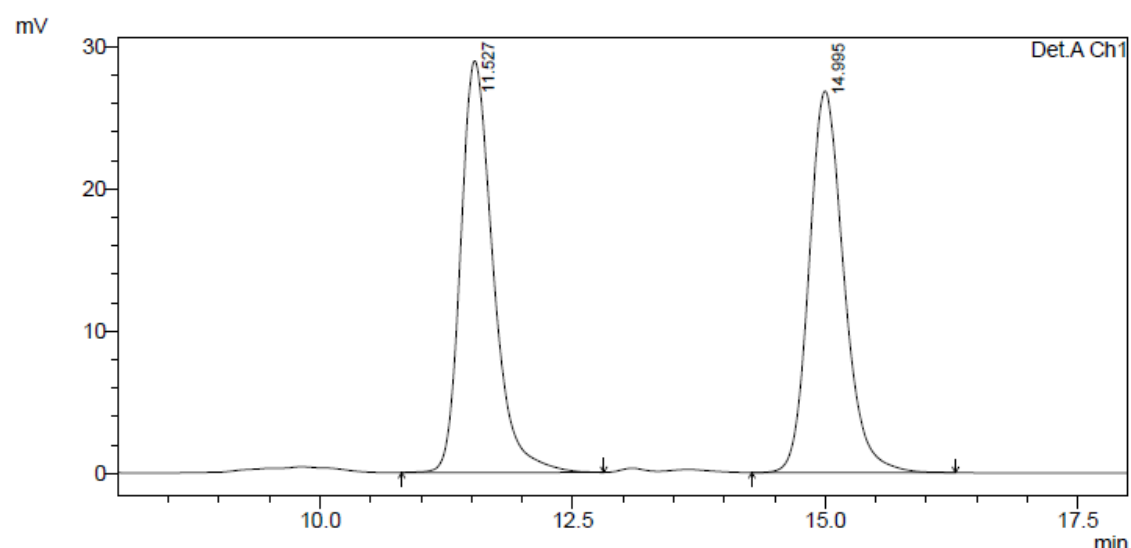

1 Det.A Ch1/254nm

Detector A Ch1 $254 \mathrm{~nm}$
\begin{tabular}{|r|r|r|r|r|r|}
\hline Peak\#\# & Ret. Time & Area & Height & Area $\%$ & \multicolumn{1}{|c|}{ Height $\%$} \\
\hline 1 & 11.527 & 647491 & 28953 & 50.140 & 51.894 \\
\hline 2 & 14.995 & 643881 & 26839 & 49.860 & 48.106 \\
\hline Total & & 1291373 & 55792 & 100.000 & 100.000 \\
\hline
\end{tabular}

$\mathrm{mV}$

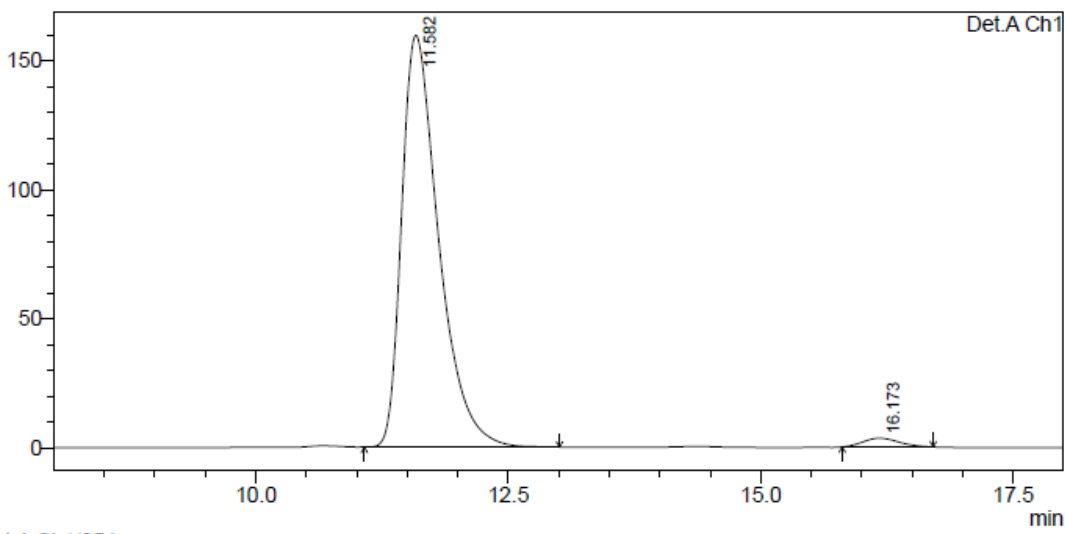

1 Det.A Ch $1 / 254 \mathrm{~nm}$

PeakTable

Detector A Ch1 $254 \mathrm{~nm}$
\begin{tabular}{|r|r|r|r|r|r|}
\hline Peak\# & Ret. Time & \multicolumn{1}{|c|}{ Area } & Height & Area $\%$ & Height $\%$ \\
\hline 1 & 11.582 & 4072246 & 159502 & 97.999 & 97.932 \\
\hline 2 & 16.173 & 83147 & 3368 & 2.001 & 2.068 \\
\hline Total & & 4155393 & 162870 & 100.000 & 100.000 \\
\hline
\end{tabular}




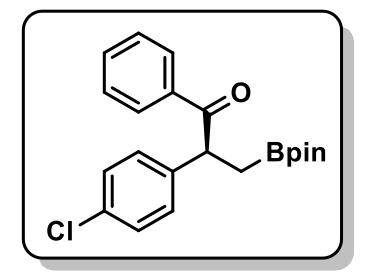

(S)-2-(4-chlorophenyl)-1-phenyl-3-(4,4,5,5-tetramethyl-1,3,2-dioxaborol

an-2-yl)propan-1-one $(3 \mathbf{i}):[\alpha]_{\mathrm{D}}{ }^{24}=+42.4,\left(\mathrm{c}=0.2, \mathrm{CH}_{2} \mathrm{Cl}_{2}\right)$. HPLC analysis (IF, Hexane/IPA = 99/1, $1 \mathrm{~mL} / \mathrm{min}, 254 \mathrm{~nm}$ ) indicated 93\% ee: $\mathrm{t}_{\mathrm{R}}$ $($ major $)=12.2 \mathrm{~min}, \mathrm{t}_{\mathrm{R}}($ minor $)=15.7 \mathrm{~min}$.

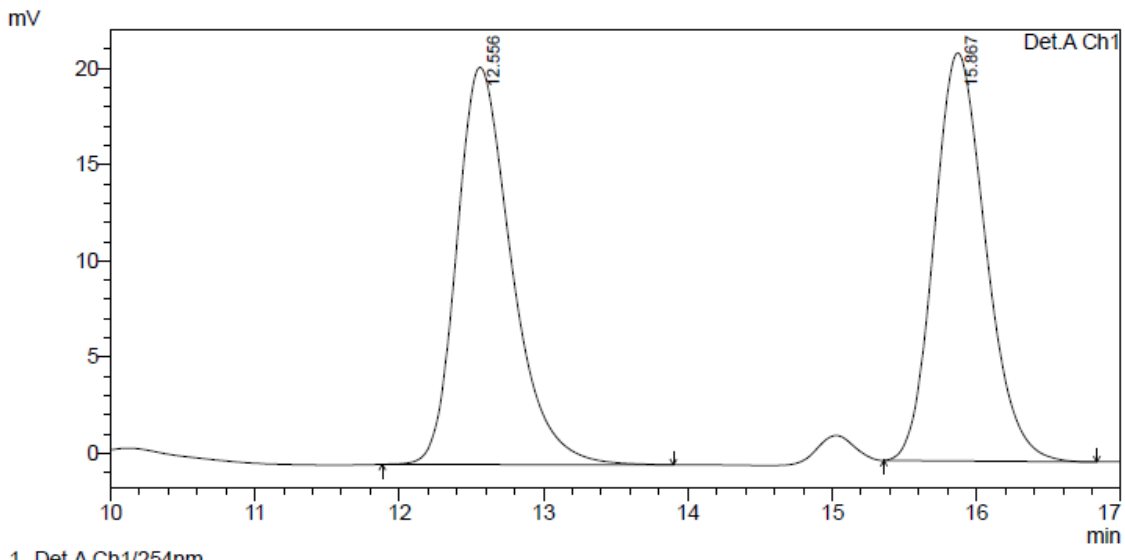

1 Det.A Ch $1 / 254 \mathrm{~nm}$

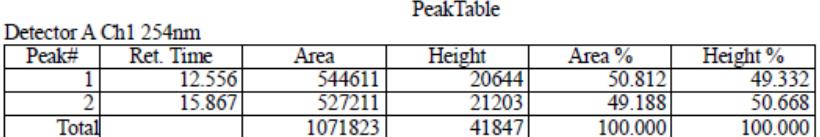

$\mathrm{mV}$

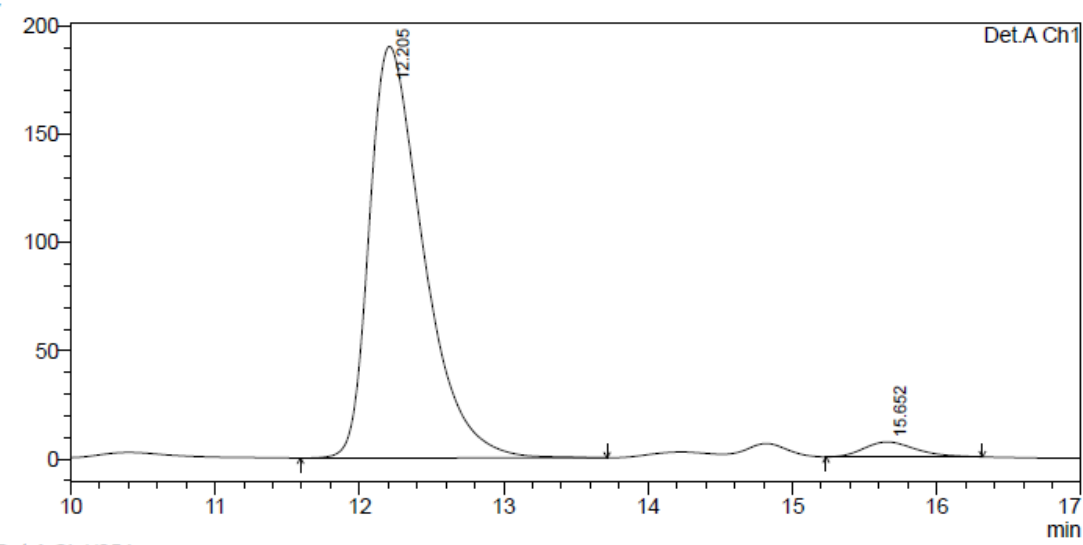

1 Det.A Ch $1 / 254 \mathrm{~nm}$

Detector A Ch1 $254 \mathrm{~nm}$
\begin{tabular}{|r|r|r|r|r|r|}
\hline Peak\# & Ret. Time & \multicolumn{1}{c|}{ Area } & Height & Area $\%$ & \multicolumn{1}{c|}{ Height $\%$} \\
\hline 1 & 12.205 & 4932685 & 190048 & 96.639 & 96.506 \\
\hline 2 & 15.652 & 171569 & 6881 & 3.361 & 3.494 \\
\hline Total & & 5104255 & 196929 & 100.000 & 100.000 \\
\hline
\end{tabular}




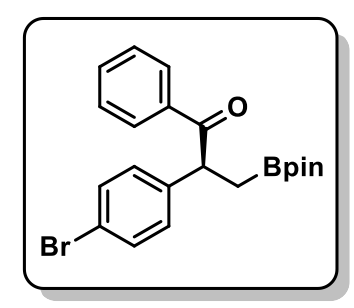

(S)-2-(4-bromophenyl)-1-phenyl-3-(4,4,5,5-tetramethyl-1,3,2-dioxaborol

an-2-yl)propan-1-one $(\mathbf{3 j}):[\alpha]_{\mathrm{D}}^{24}=+49.7,\left(\mathrm{c}=0.2, \mathrm{CH}_{2} \mathrm{Cl}_{2}\right)$. HPLC analysis (IF, Hexane/IPA = 99/1, $1 \mathrm{~mL} / \mathrm{min}, 254 \mathrm{~nm}$ ) indicated 97\% ee: $\mathrm{t}_{\mathrm{R}}$ $($ major $)=13.3 \min , \mathrm{t}_{\mathrm{R}}($ minor $)=19.0 \mathrm{~min}$.

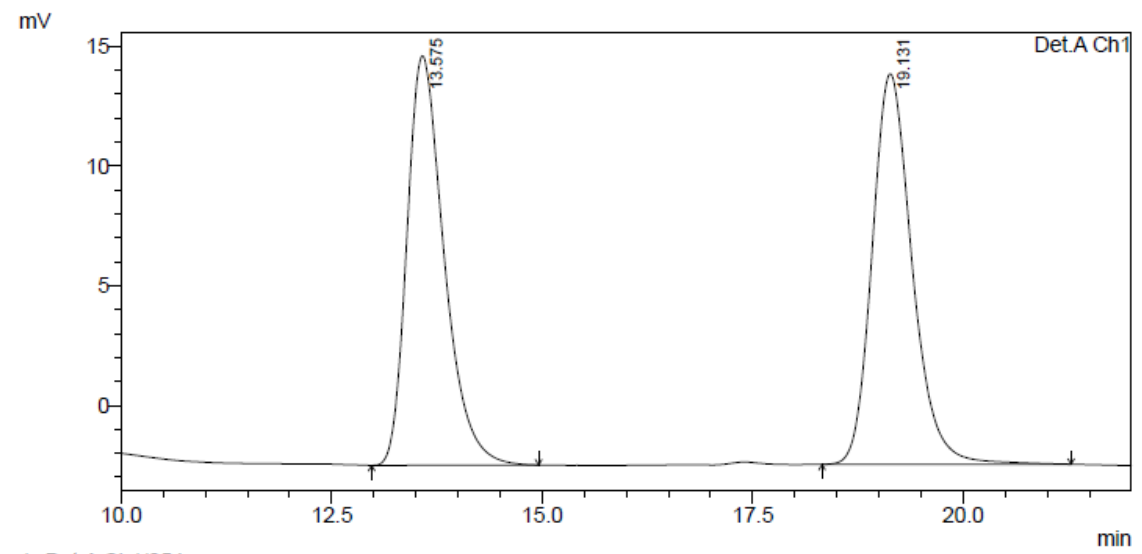

1 Det.ACh $1 / 254 \mathrm{~nm}$

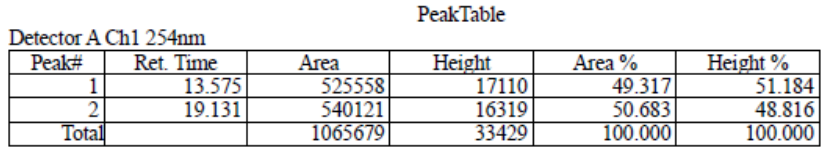

$\mathrm{mV}$

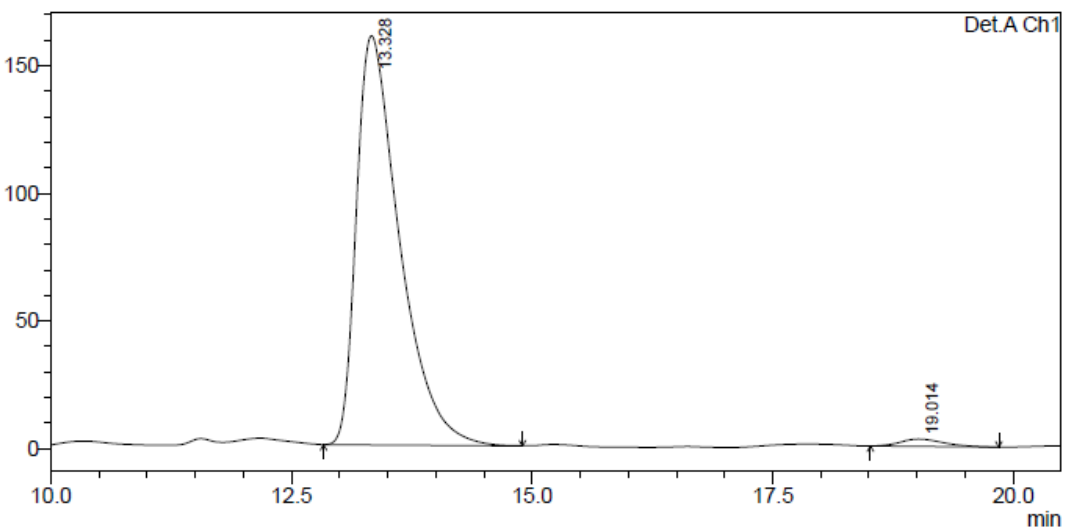

1 Det.A Ch1/254nm

PeakTable

Detector A Ch1 $254 \mathrm{~nm}$
\begin{tabular}{|r|r|r|r|r|r|}
\hline Peak\# & Ret. Time & \multicolumn{1}{|c|}{ Area } & \multicolumn{1}{|c|}{ Height } & Area $\%$ & \multicolumn{1}{c|}{ Height \% } \\
\hline 1 & 13.328 & 5006182 & 160379 & 98.253 & 98.244 \\
\hline 2 & 19.014 & 89019 & 2867 & 1.747 & 1.756 \\
\hline Total & & 5095202 & 163246 & 100.000 & 100.000 \\
\hline
\end{tabular}




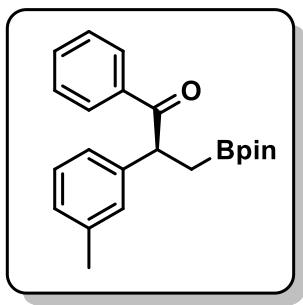

(S)-1-phenyl-3-(4,4,5,5-tetramethyl-1,3,2-dioxaborolan-2-yl)-2-(m-tolyl)pr opan-1-one (3k): $[\alpha]_{\mathrm{D}}{ }^{24}=+59.8,\left(\mathrm{c}=0.2, \mathrm{CH}_{2} \mathrm{Cl}_{2}\right)$. HPLC analysis (IF, Hexane/IPA $=99 / 1,1 \mathrm{~mL} / \mathrm{min}, 254 \mathrm{~nm})$ indicated 99\% ee: $\mathrm{t}_{\mathrm{R}}($ major $)=10.0$ $\min , \mathrm{t}_{\mathrm{R}}($ minor $)=13.4 \mathrm{~min}$.

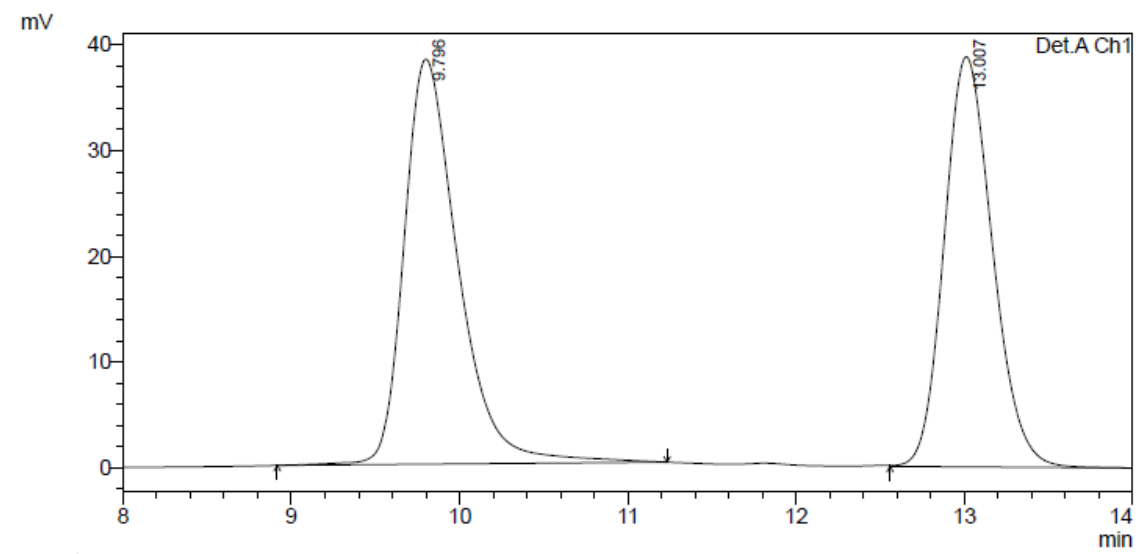

1 Det.A Ch $1 / 254 \mathrm{~nm}$
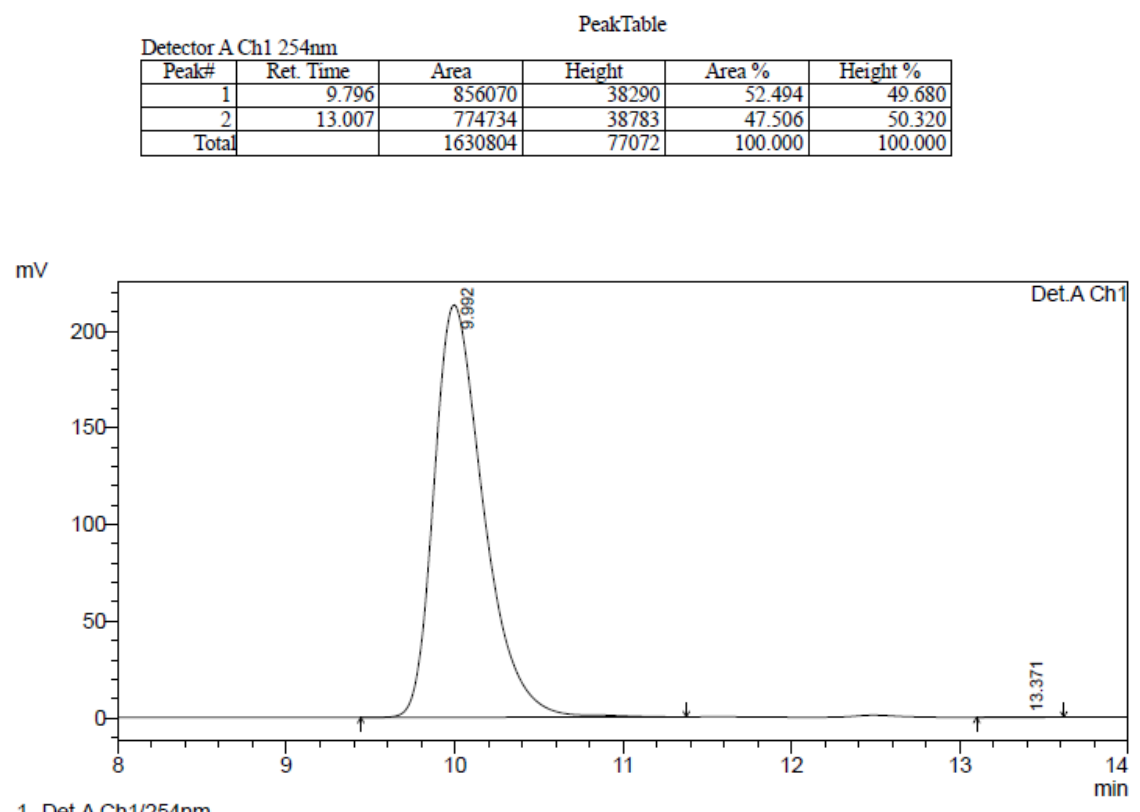

1 Det.A Ch1/254nm

PeakTable

Detector A Ch $1254 \mathrm{~nm}$
\begin{tabular}{|r|r|r|r|r|r|}
\hline Peak\# & Ret. Time & \multicolumn{1}{c|}{ Area } & Height & Area $\%$ & Height $\%$ \\
\hline 1 & 9.992 & 4370724 & 213355 & 99.948 & 99.930 \\
\hline 2 & 13.371 & 2280 & 149 & 0.052 & 0.070 \\
\hline Total| & & 4373004 & 213504 & 100.000 & 100.000 \\
\hline
\end{tabular}




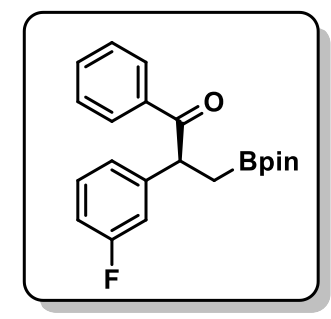

(S)-2-(3-fluorophenyl)-1-phenyl-3-(4,4,5,5-tetramethyl-1,3,2-dioxaborola

n-2-yl)propan-1-one (3I): $[\alpha]_{\mathrm{D}}{ }^{24}=+27.2,\left(\mathrm{c}=0.2, \mathrm{CH}_{2} \mathrm{Cl}_{2}\right)$. HPLC analysis (IF, Hexane/IPA $=99 / 1,1 \mathrm{~mL} / \mathrm{min}, 254 \mathrm{~nm})$ indicated 98\% ee: $\mathrm{t}_{\mathrm{R}}($ major $)=$ $12.3 \min , \mathrm{t}_{\mathrm{R}}(\operatorname{minor})=15.9 \min$.

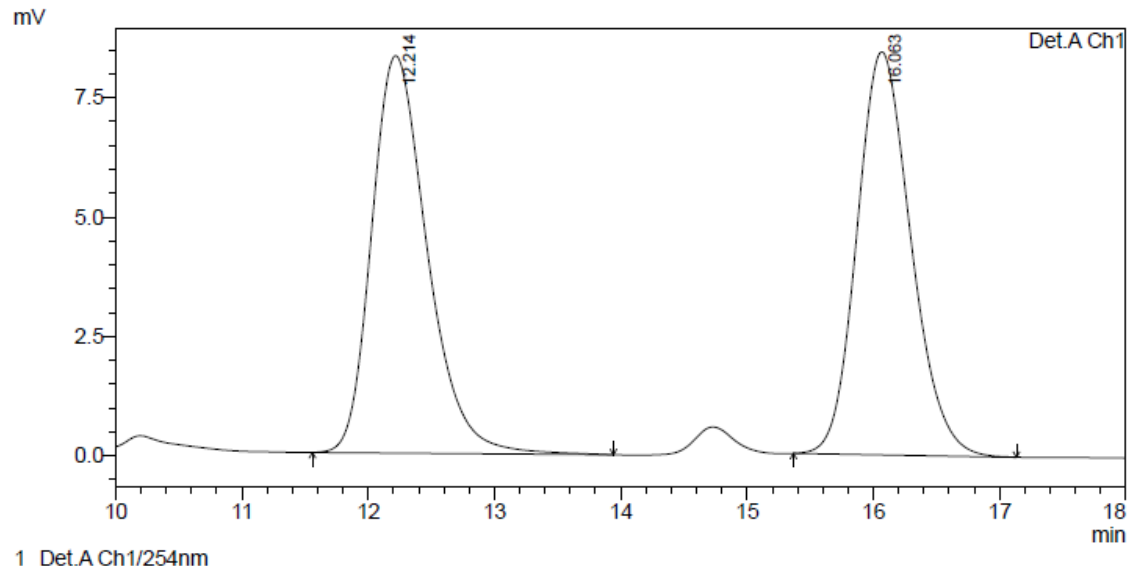

1 Det.A Ch1/254nm

Detector A Ch1 $254 \mathrm{~nm}$
\begin{tabular}{|r|r|r|r|r|r|}
\hline Peak\# & Ret. Time & Area & Height & Area \% & Height \% \\
\hline 1 & 12.214 & 255251 & 8327 & 50.404 & 49.650 \\
\hline 2 & 16.063 & 251161 & 8445 & 49.596 & 50.350 \\
\hline Total & & 506413 & 16772 & 100.000 & 100.000 \\
\hline
\end{tabular}

$\mathrm{mV}$

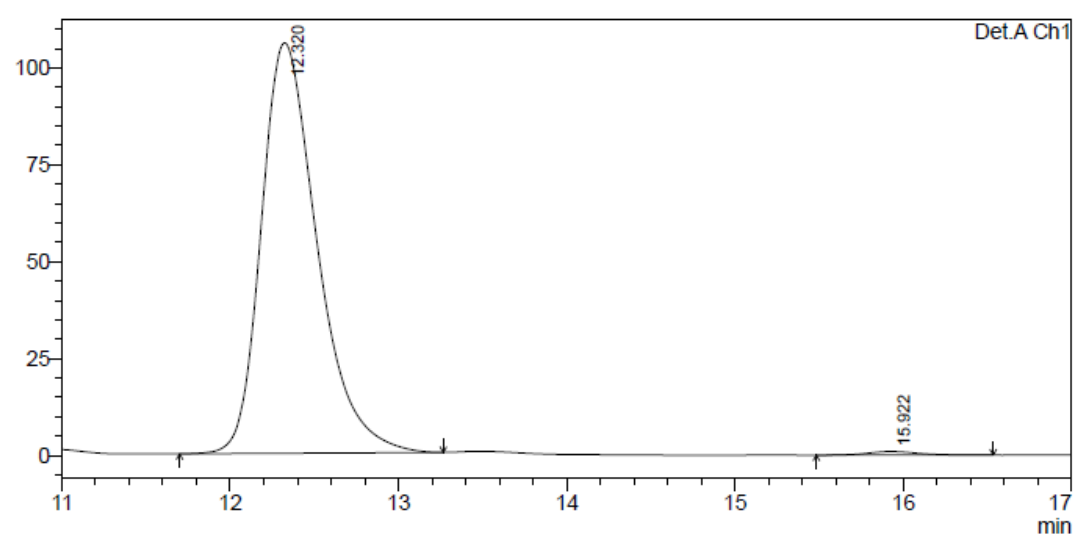

1 Det.ACh $1 / 254 \mathrm{~nm}$

Detector A Ch1 $254 \mathrm{~nm}$
\begin{tabular}{|r|r|r|r|r|r|}
\hline Peak\# & Ret. Time & \multicolumn{1}{c|}{ Area } & Height & Area $\%$ & \multicolumn{1}{c|}{ Height $\%$} \\
\hline 1 & 12.320 & 2444330 & 105905 & 99.217 & 99.118 \\
\hline 2 & 15.922 & 19297 & 943 & 0.783 & 0.882 \\
\hline Total & & 2463627 & 106848 & 100.000 & 100.000 \\
\hline
\end{tabular}


n-2-yl)propan-1-one (3m): $[\alpha]_{\mathrm{D}}{ }^{24}=+7.6,\left(\mathrm{c}=0.2, \mathrm{CH}_{2} \mathrm{Cl}_{2}\right)$. HPLC analysis (IF, Hexane/IPA $=99 / 1,1 \mathrm{~mL} / \mathrm{min}, 254 \mathrm{~nm})$ indicated $70 \%$ ee: $\mathrm{t}_{\mathrm{R}}($ major $)=$ $10.6 \min , \mathrm{t}_{\mathrm{R}}(\operatorname{minor})=14.3 \mathrm{~min}$.

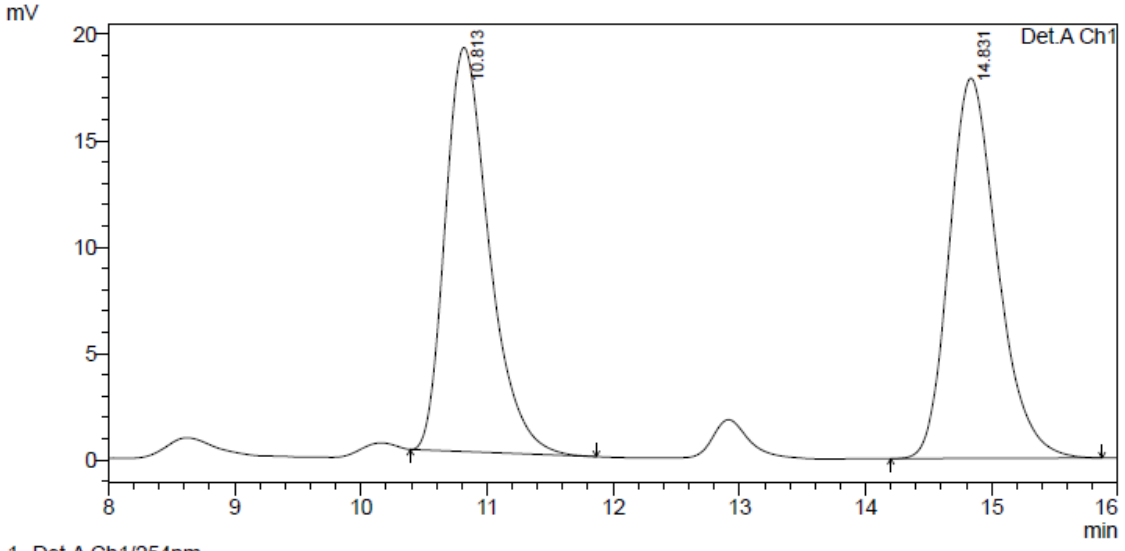

1 Det.A Ch1/254nm

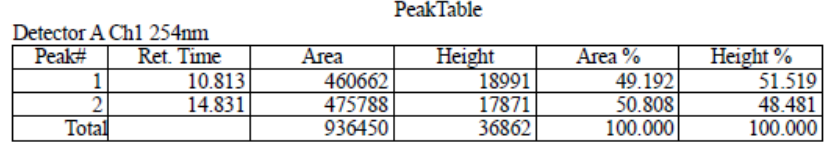

$\mathrm{mV}$

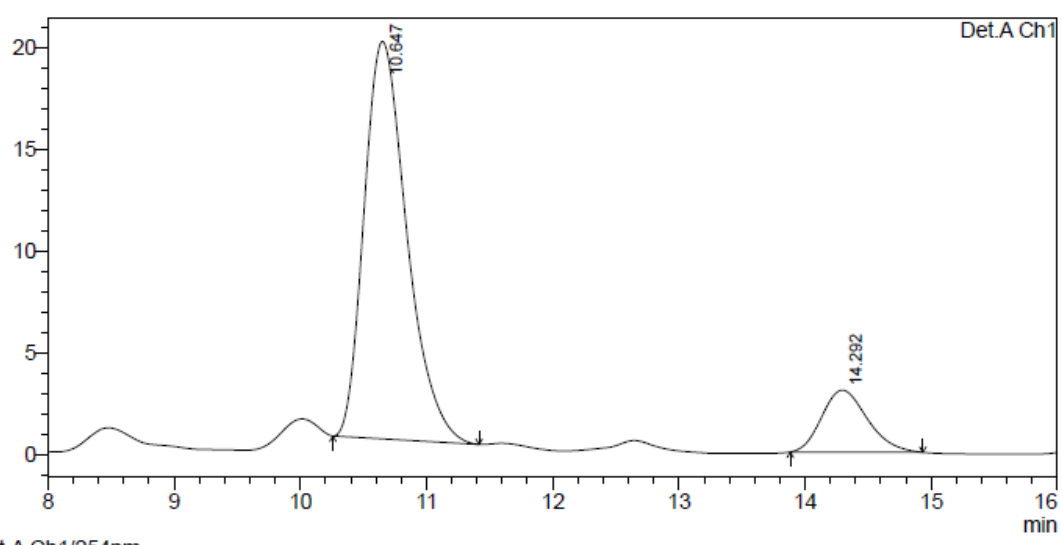

1 Det.A Ch1/254nm

Detector A Ch1 $254 \mathrm{~nm}$
\begin{tabular}{|r|r|r|r|r|r|}
\hline \multicolumn{1}{|c|}{ Peak\# } & Ret. Time & \multicolumn{1}{c|}{ Area } & Height & Area $\%$ & Height $\%$ \\
\hline 1 & 10.647 & 461510 & 19568 & 85.740 & 86.513 \\
\hline 2 & 14.292 & 76755 & 3051 & 14.260 & 13.487 \\
\hline Total & & 538265 & 22618 & 100.000 & 100.000 \\
\hline
\end{tabular}




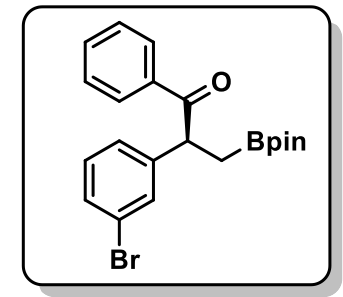

(S)-2-(3-bromophenyl)-1-phenyl-3-(4,4,5,5-tetramethyl-1,3,2-dioxaborol an-2-yl)propan-1-one (3n): $[\alpha]_{\mathrm{D}}{ }^{24}=+18.2,\left(\mathrm{c}=0.2, \mathrm{CH}_{2} \mathrm{Cl}_{2}\right)$. HPLC analysis (IF, Hexane/IPA = 99/1, $1 \mathrm{~mL} / \mathrm{min}, 254 \mathrm{~nm})$ indicated $74 \%$ ee: $\mathrm{t}_{\mathrm{R}}$ $($ major $)=12.5 \mathrm{~min}, \mathrm{t}_{\mathrm{R}}($ minor $)=16.5 \mathrm{~min}$.

$\mathrm{mV}$

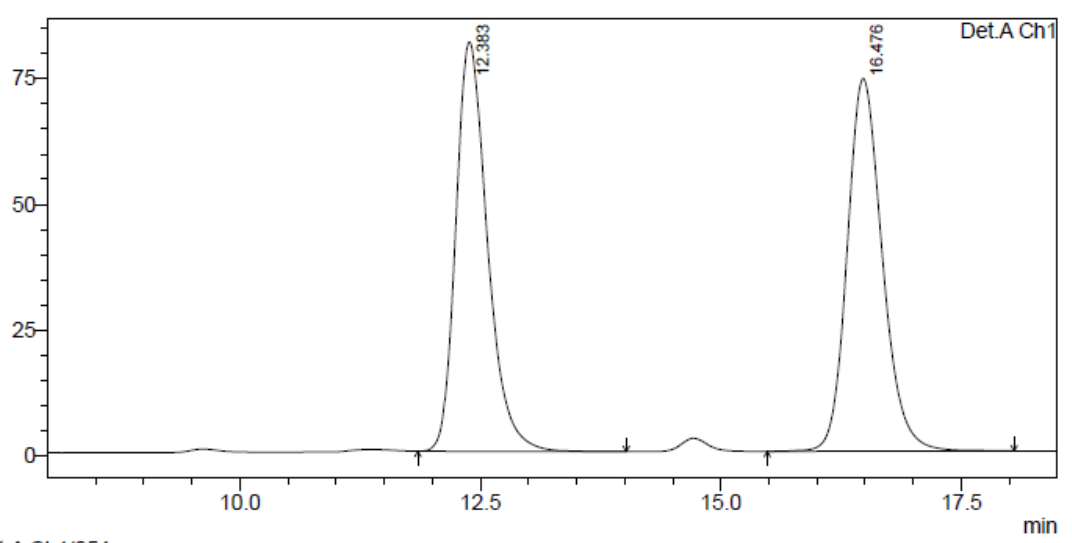

1 Det.A Ch1/254nm

PeakTable

Detector A Ch1 $254 \mathrm{~nm}$
\begin{tabular}{|r|r|r|r|r|r|}
\hline Peak\# & Ret. Time & \multicolumn{1}{c|}{ Area } & \multicolumn{1}{|c|}{ Height } & Area $\%$ & \multicolumn{1}{c|}{ Height $\%$} \\
\hline 1 & 12.383 & 1870600 & 81553 & 49.740 & 52.343 \\
\hline 2 & 16.476 & 1890143 & 74254 & 50.260 & 47.657 \\
\hline Total & & 3760743 & 155807 & 100.000 & 100.000 \\
\hline
\end{tabular}

$\mathrm{mV}$

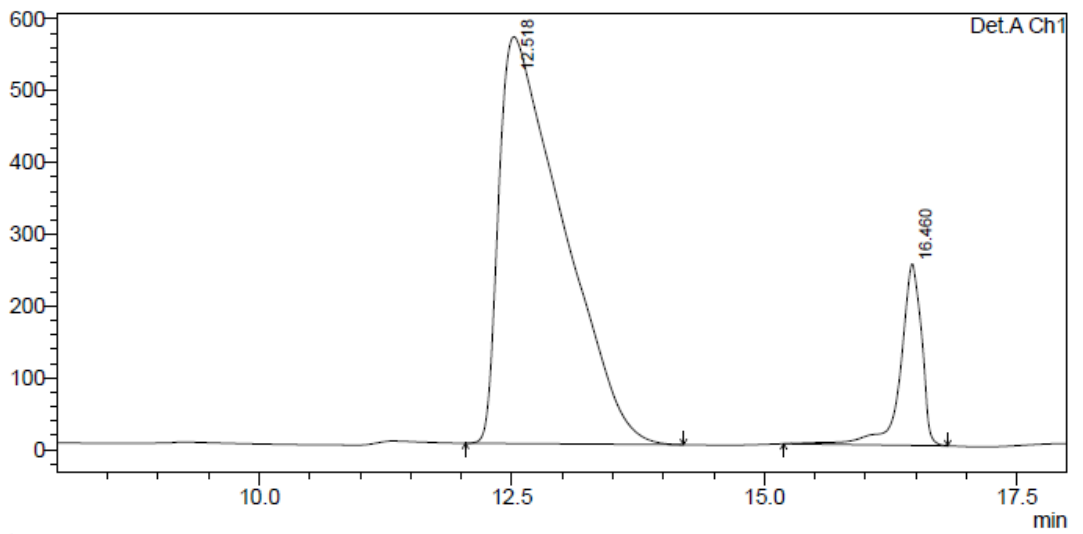

1 Det.ACh $1 / 254 \mathrm{~nm}$

PeakTable

Detector A Ch1 $254 \mathrm{~nm}$
\begin{tabular}{|r|r|r|r|r|r|}
\hline Peak\# & Ret. Time & \multicolumn{1}{|c|}{ Area } & Height & Area \% & \multicolumn{1}{c|}{ Height \% } \\
\hline 1 & 12.518 & 24941686 & 566341 & 87.093 & 69.150 \\
\hline 2 & 16.460 & 3696167 & 252660 & 12.907 & 30.850 \\
\hline Total & & 28637852 & 819001 & 100.000 & 100.000 \\
\hline
\end{tabular}




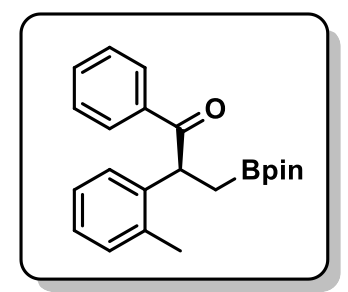

(S)-1-phenyl-3-(4,4,5,5-tetramethyl-1,3,2-dioxaborolan-2-yl)-2-(o-tolyl) propan-1-one (3o): $[\alpha]_{D}^{24}=+103.1,\left(\mathrm{c}=0.2, \mathrm{CH}_{2} \mathrm{Cl}_{2}\right)$. HPLC analysis (IA, Hexane/IPA $=99 / 1,1 \mathrm{~mL} / \mathrm{min}, 254 \mathrm{~nm})$ indicated $93 \%$ ee: $\mathrm{t}_{\mathrm{R}}($ major $)=6.7$ $\min , t_{R}(\operatorname{minor})=8.7 \mathrm{~min}$.

$\mathrm{mV}$

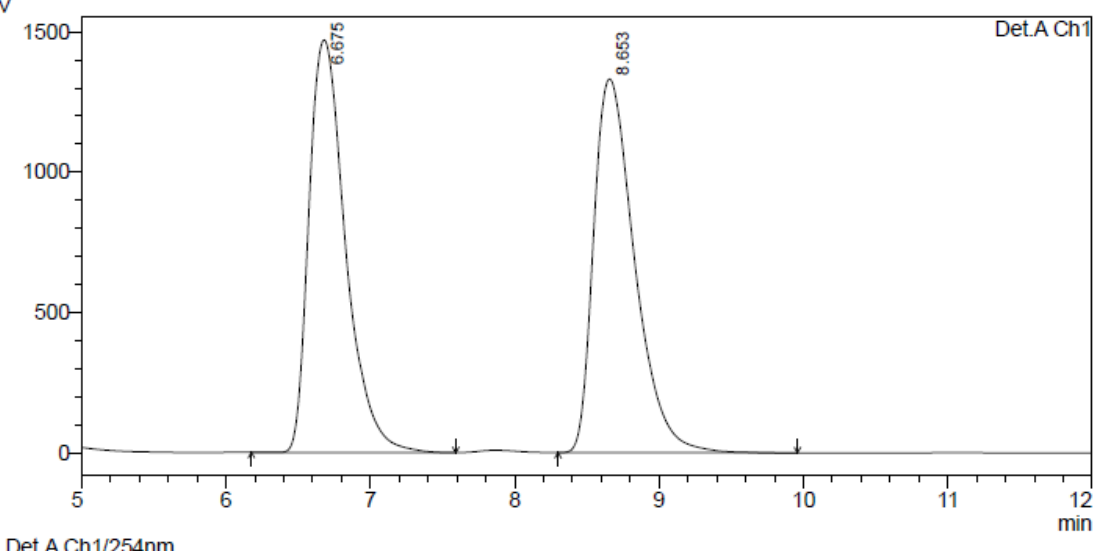

1 Det.A Ch $1 / 254 \mathrm{~nm}$

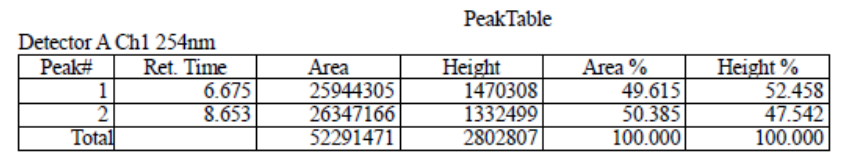

$\mathrm{mV}$

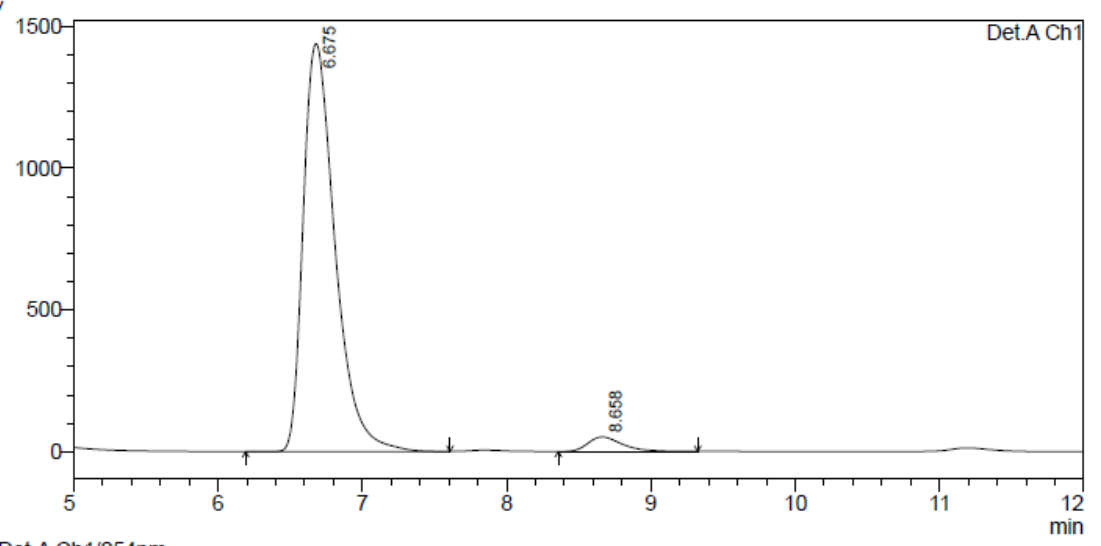

1 Det.A Ch1/254nm

Detector A Ch1 $254 \mathrm{~nm}$
\begin{tabular}{|r|r|r|r|r|r|}
\hline \multicolumn{1}{|c|}{ Peak\# } & \multicolumn{1}{|c|}{ Ret. Time } & \multicolumn{1}{c|}{ Area } & Height & Area $\%$ & Height $\%$ \\
\hline 1 & 6.675 & 22110785 & 1438153 & 96.336 & 96.579 \\
\hline 2 & 8.658 & 840904 & 50948 & 3.664 & 3.421 \\
\hline Total & & 22951689 & 1489102 & 100.000 & 100.000 \\
\hline
\end{tabular}




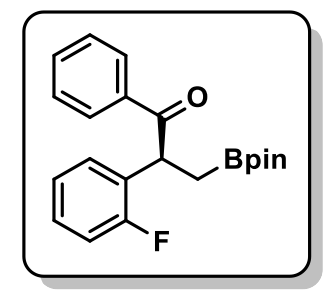

(S)-2-(2-fluorophenyl)-1-phenyl-3-(4,4,5,5-tetramethyl-1,3,2-dioxaborolan

-2-yl)propan-1-one (3p): $[\alpha]_{\mathrm{D}}{ }^{24}=+31.0,\left(\mathrm{c}=0.2, \mathrm{CH}_{2} \mathrm{Cl}_{2}\right)$. HPLC analysis $(\mathrm{IF}$, Hexane/IPA $=99 / 1,1 \mathrm{~mL} / \mathrm{min}, 254 \mathrm{~nm})$ indicated $93 \%$ ee: $\mathrm{t}_{\mathrm{R}}($ major $)=$ $9.1 \mathrm{~min}, \mathrm{t}_{\mathrm{R}}($ minor $)=12.2 \mathrm{~min}$.

$\mathrm{m} \vee$

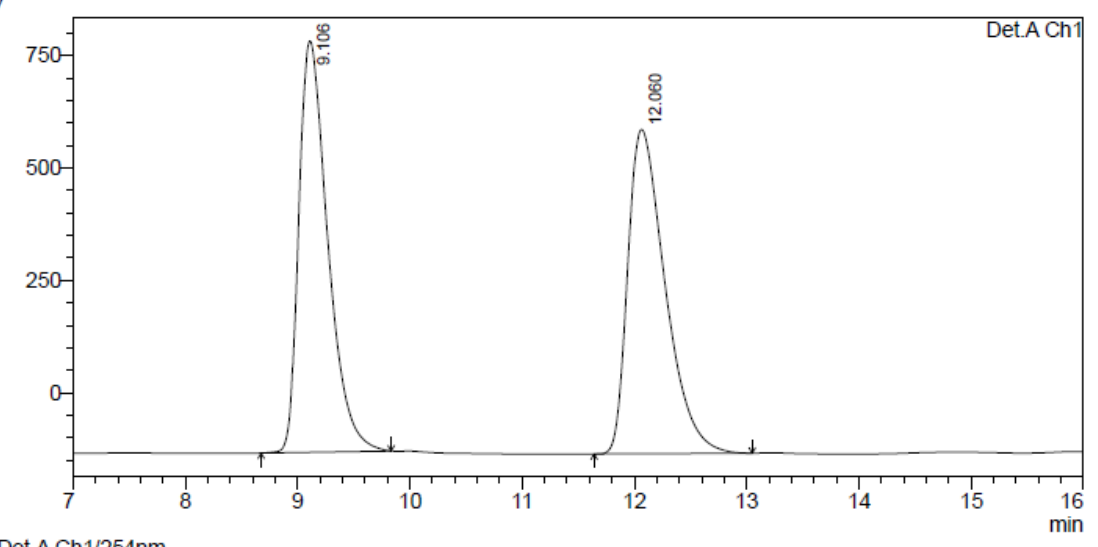

1 Det.ACh $1 / 254 \mathrm{~nm}$

Detector A Ch1 $254 \mathrm{~nm}$
\begin{tabular}{|r|r|r|r|r|r|}
\hline Peak\# & Ret. Time & \multicolumn{1}{|c|}{ Area } & Height & Area \% & \multicolumn{1}{c|}{ Height \% } \\
\hline 1 & 9.106 & 16431409 & 914899 & 49.621 & 55.924 \\
\hline 2 & 12.060 & 16682133 & 721066 & 50.379 & 44.076 \\
\hline Total & & 33113542 & 1635965 & 100.000 & 100.000 \\
\hline
\end{tabular}

$\mathrm{mV}$

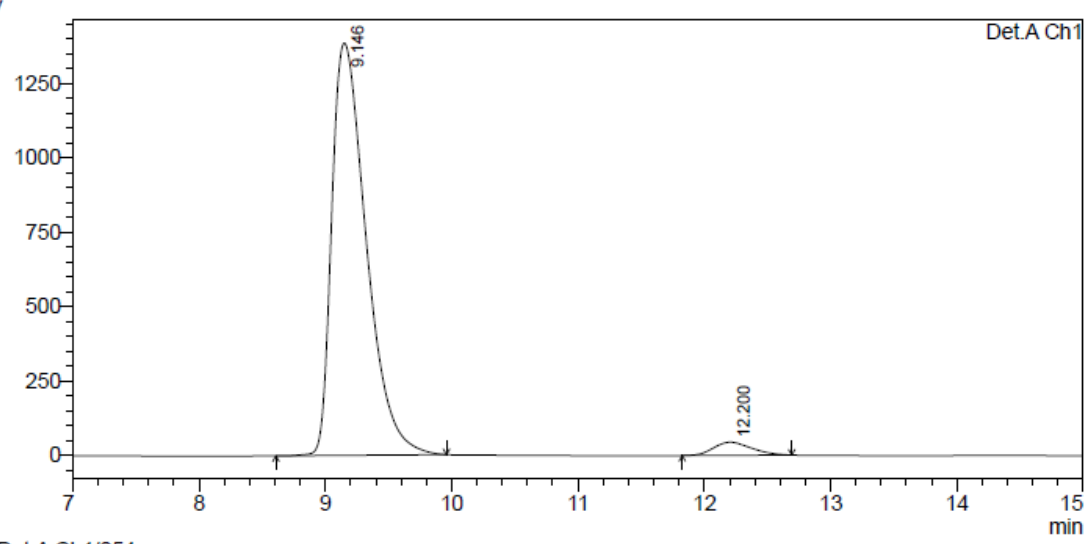

1 Det.A Ch1/254nm

PeakTable
\begin{tabular}{|r|r|r|r|r|r|}
\hline Detector A Ch1 254nm \\
\hline Peak\# & Ret. Time & \multicolumn{1}{|c|}{ Area } & Height & Area \% & \multicolumn{1}{c|}{ Height \% } \\
\hline 1 & 9.146 & 26300676 & 1385690 & 96.622 & 96.924 \\
\hline 2 & 12.200 & 919523 & 43971 & 3.378 & 3.076 \\
\hline Total| & & 27220200 & 1429661 & 100.000 & 100.000 \\
\hline
\end{tabular}




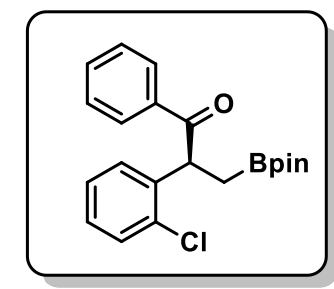

(S)-2-(2-chlorophenyl)-1-phenyl-3-(4,4,5,5-tetramethyl-1,3,2-dioxaborola n-2-yl)propan-1-one (3q): $[\alpha]_{\mathrm{D}}{ }^{24}=+12.8,\left(\mathrm{c}=0.2, \mathrm{CH}_{2} \mathrm{Cl}_{2}\right)$. HPLC analysis $(\mathrm{IA}$, Hexane $/ \mathrm{IPA}=99 / 1,1 \mathrm{~mL} / \mathrm{min}, 254 \mathrm{~nm})$ indicated $84 \%$ ee: $\mathrm{t}_{\mathrm{R}}($ major $)=$ $10.8 \min , \mathrm{t}_{\mathrm{R}}($ minor $)=13.4 \mathrm{~min}$.

$\mathrm{mV}$

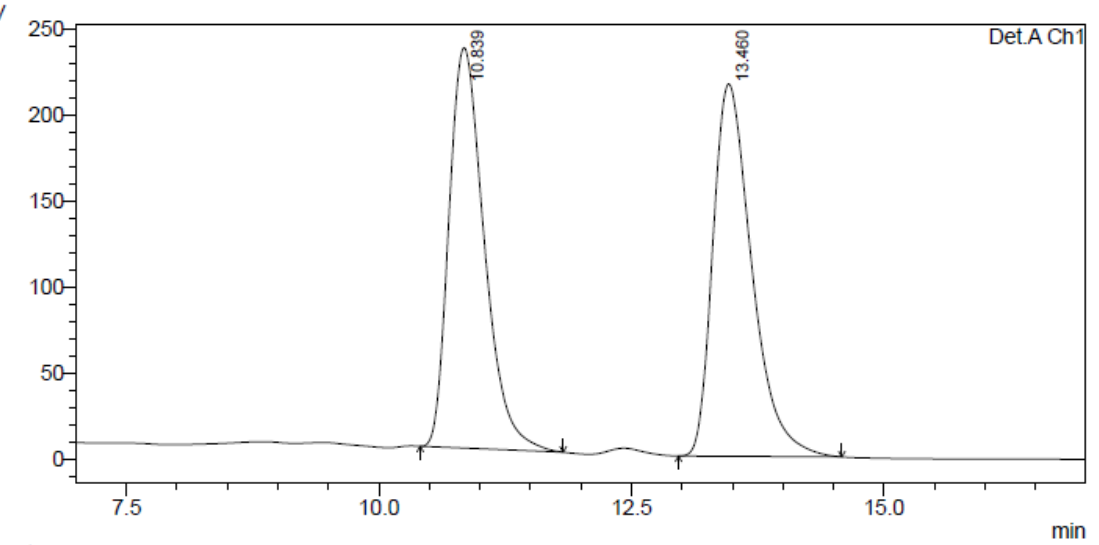

1 Det.A Ch $1 / 254 \mathrm{~nm}$

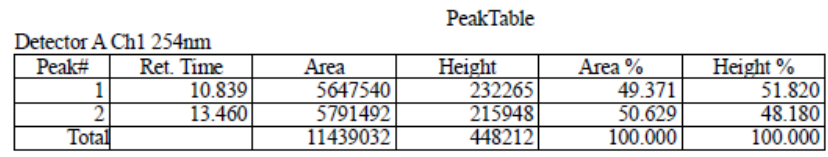

$\mathrm{mV}$

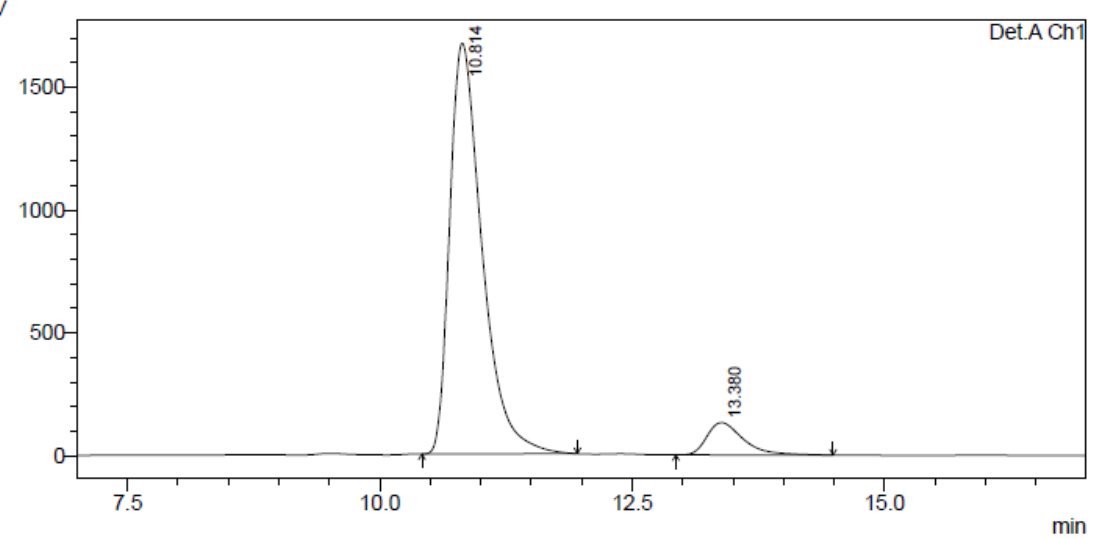

1 Det.A Ch1/254nm

PeakTable

Detector A Ch1 $254 \mathrm{~nm}$
\begin{tabular}{|r|r|r|r|r|r|}
\hline \multicolumn{1}{|c|}{ Peak\#\# } & Ret. Time & \multicolumn{1}{|c|}{ Area } & \multicolumn{1}{c|}{ Height } & Area $\%$ & Height \% \\
\hline 1 & 10.814 & 37290891 & 1670881 & 91.863 & 92.680 \\
\hline 2 & 13.380 & 3303243 & 131962 & 8.137 & 7.320 \\
\hline Total & & 40594134 & 1802843 & 100.000 & 100.000 \\
\hline
\end{tabular}




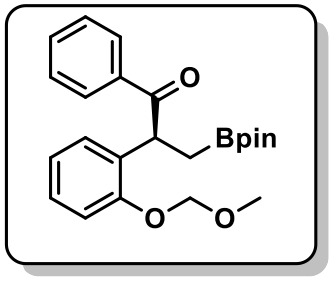

(S)-2-(2-(methoxymethoxy)phenyl)-1-phenyl-3-(4,4,5,5-tetramethyl-1,3,2

-dioxaborolan-2-yl)propan-1-one $(3 \mathbf{r}):[\alpha]_{\mathrm{D}}^{24}=+9.1,\left(\mathrm{c}=0.2, \mathrm{CH}_{2} \mathrm{Cl}_{2}\right)$. HPLC analysis (IF, Hexane/IPA = 99/1, $1 \mathrm{~mL} / \mathrm{min}, 254 \mathrm{~nm}$ ) indicated 95\% ee: $t_{R}($ major $)=9.3 \mathrm{~min}, t_{R}($ minor $)=10.8 \min$.

$\mathrm{mV}$

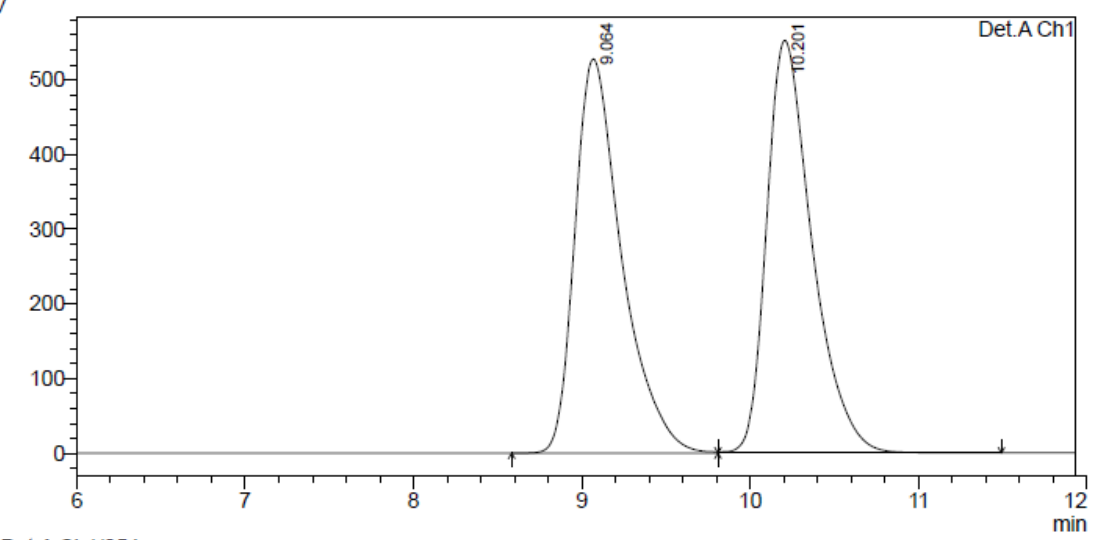

1 Det.A Ch $1 / 254 \mathrm{~nm}$

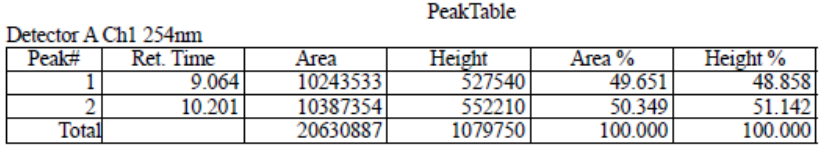

$\mathrm{mV}$

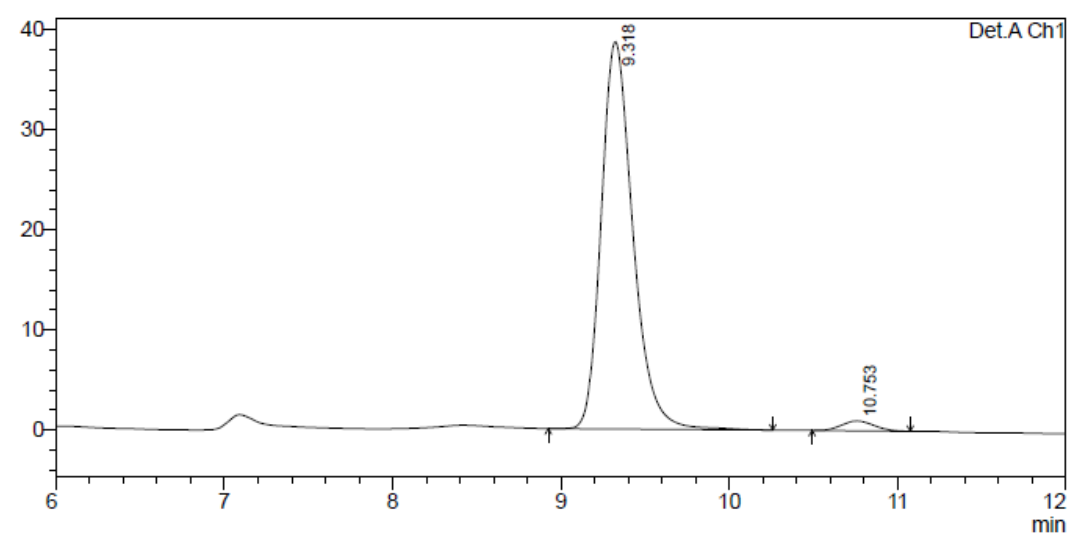

1 Det.A Ch $1 / 254 \mathrm{~nm}$

Detector A Ch1 $254 \mathrm{~nm}$
\begin{tabular}{|r|r|r|r|r|r|}
\hline Peak\# & Ret. Time & \multicolumn{1}{c|}{ Area } & Height & Area $\%$ & Height $\%$ \\
\hline 1 & 9.318 & 507560 & 38702 & 97.494 & 97.532 \\
\hline 2 & 10.753 & 13046 & 979 & 2.506 & 2.468 \\
\hline Total & & 520606 & 39681 & 100.000 & 100.000 \\
\hline
\end{tabular}




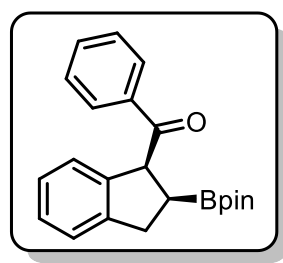

phenyl(2-(4,4,5,5-tetramethyl-1,3,2-dioxaborolan-2-yl)-2,3-dihydro-1H-ind

en-1-yl)methanone (3s): $[\alpha]_{\mathrm{D}}{ }^{24}=+6.5,\left(\mathrm{c}=0.2, \mathrm{CH}_{2} \mathrm{Cl}_{2}\right)$. HPLC analysis $(\mathrm{OD}-\mathrm{H}$, Hexane/IPA $=99 / 1,1 \mathrm{~mL} / \mathrm{min}, 254 \mathrm{~nm})$ indicated $97 \%$ ee: $\mathrm{t}_{\mathrm{R}}$ (minor) $=11.2 \mathrm{~min}, \mathrm{t}_{\mathrm{R}}($ major $)=12.2 \mathrm{~min}$.

$\mathrm{mV}$

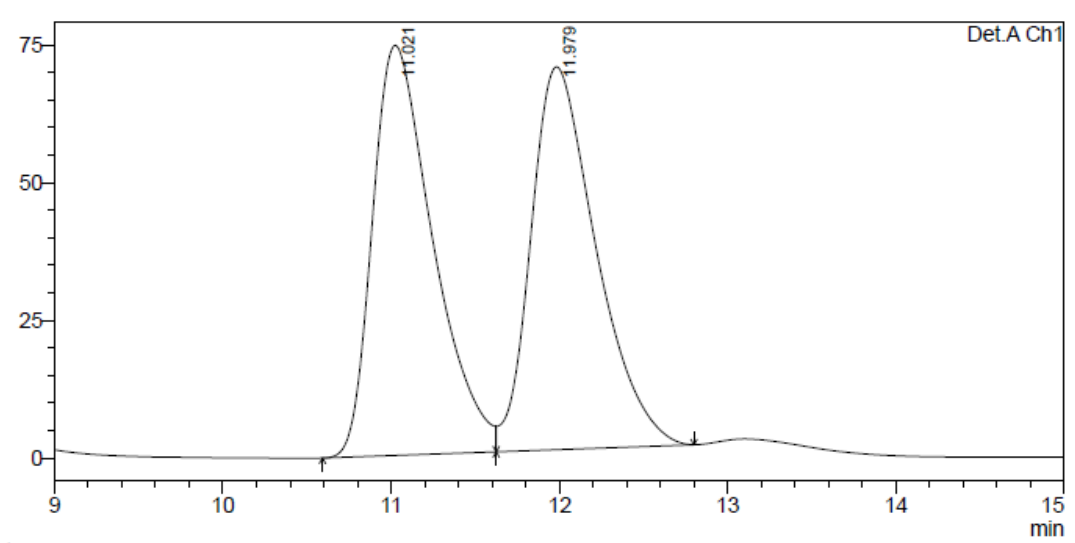

1 Det.A Ch1/254nm
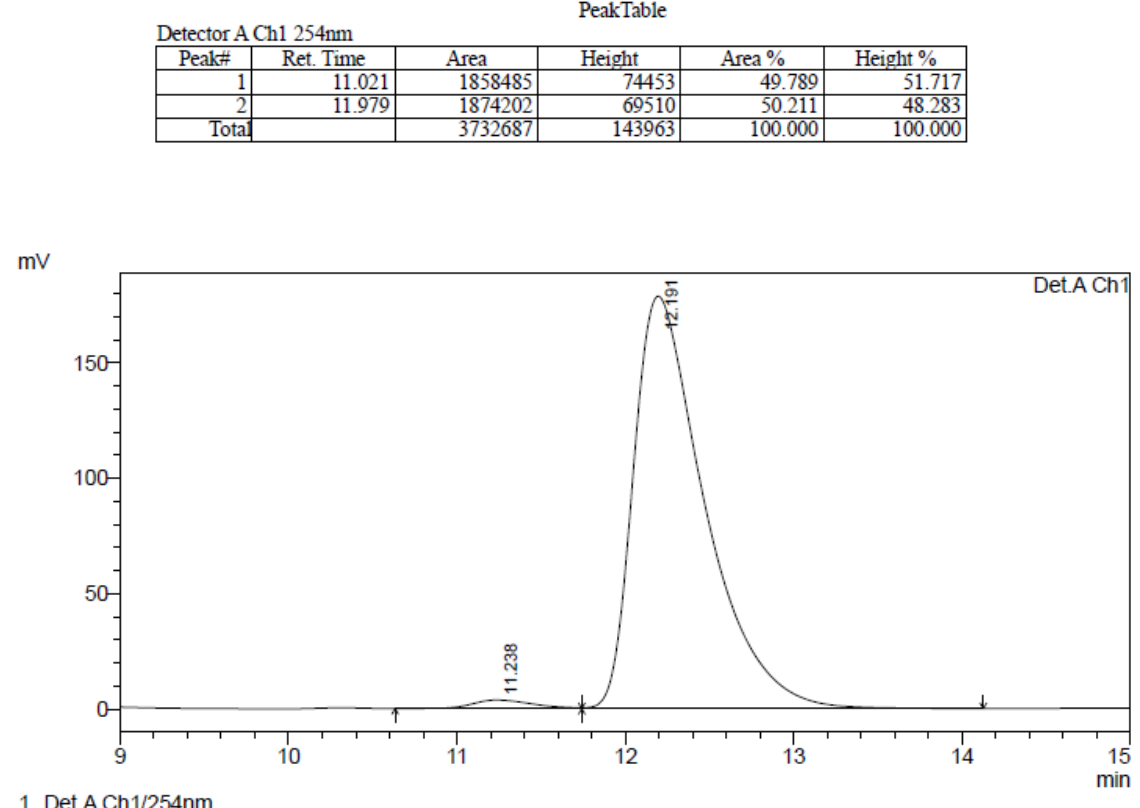

1 Det.A Ch1/254nm

Detector A Ch1 254nm
\begin{tabular}{|r|r|r|r|r|r|}
\hline Peak\# & Ret. Time & \multicolumn{1}{c|}{ Area } & Height & Area $\%$ & \multicolumn{1}{c|}{ Height $\%$} \\
\hline 1 & 11.238 & 92783 & 3775 & 1.738 & 2.070 \\
\hline 2 & 12.191 & 5246856 & 178564 & 98.262 & 97.930 \\
\hline Total & & 5339639 & 182339 & 100.000 & 100.000 \\
\hline
\end{tabular}




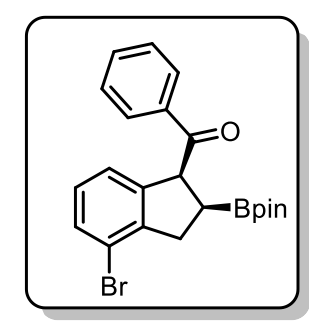

$\mathrm{mV}$

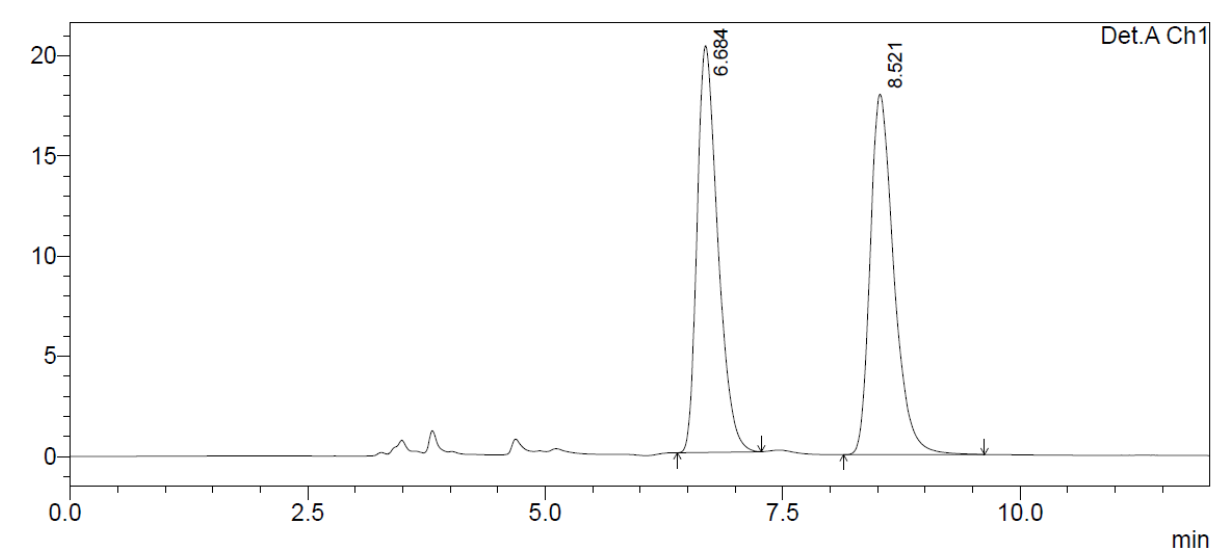

1 Det.A Ch1/254nm

Detector A Ch1 254nm

Detector A Ch1 $254 \mathrm{~nm}$
\begin{tabular}{|r|r|r|r|r|r|}
\hline \multicolumn{1}{|c|}{ Peak\# } & Ret. Time & \multicolumn{1}{|c|}{ Area } & Height & Area $\%$ & \multicolumn{1}{|c|}{ Height $\%$} \\
\hline 1 & 6.684 & 308494 & 20297 & 49.650 & 53.015 \\
\hline 2 & 8.521 & 312841 & 17989 & 50.350 & 46.985 \\
\hline Total & & 621335 & 38286 & 100.000 & 100.000 \\
\hline
\end{tabular}

$\mathrm{mV}$

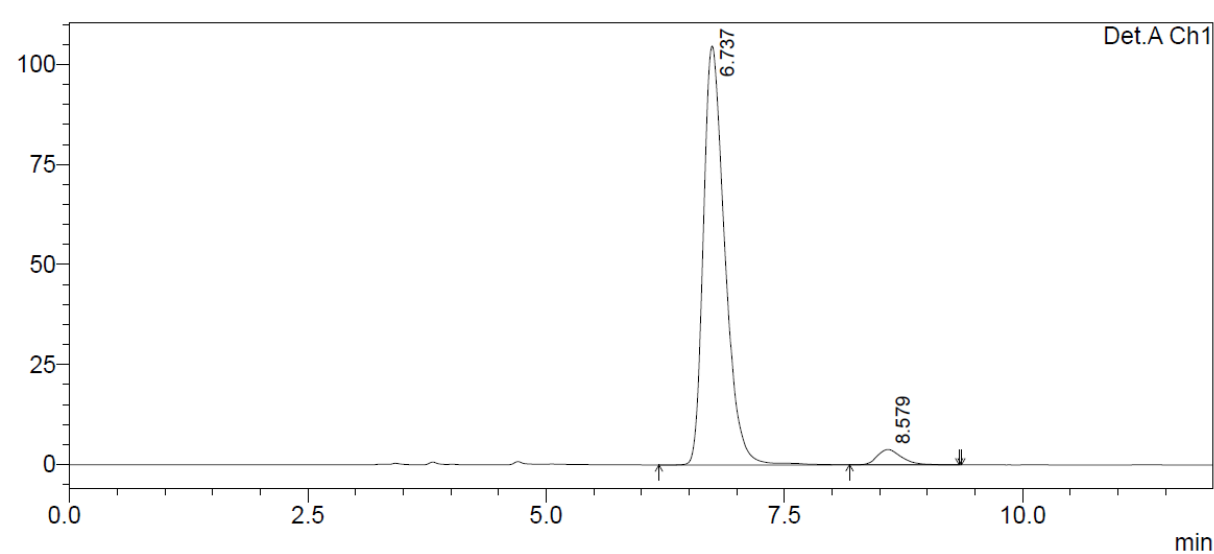

1 Det.A Ch1/254nm

PeakTable

Detector A Ch1 $254 \mathrm{~nm}$
\begin{tabular}{|r|r|r|r|r|r|}
\hline Peak\# & Ret. Time & \multicolumn{1}{|c|}{ Area } & Height & Area $\%$ & Height $\%$ \\
\hline 1 & 6.737 & 1640338 & 104711 & 96.086 & 96.489 \\
\hline 2 & 8.579 & 66822 & 3810 & 3.914 & 3.511 \\
\hline Total & & 1707161 & 108522 & 100.000 & 100.000 \\
\hline
\end{tabular}




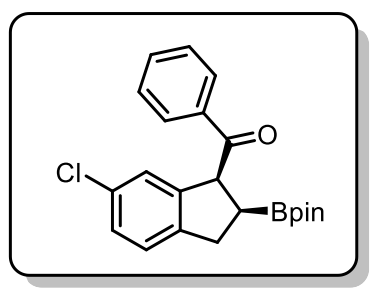

((1S,2S)-6-chloro-2-(4,4,5,5-tetramethyl-1,3,2-dioxaborolan-2-yl)-2,3-

dihydro-1H-inden-1-yl)(phenyl)methanone $(3 \mathrm{u}):[\alpha]_{\mathrm{D}}^{24}=+21.6,(\mathrm{c}=$ 0.2, $\mathrm{CH}_{2} \mathrm{Cl}_{2}$ ). HPLC analysis (IF, Hexane/IPA = 99/1, $1 \mathrm{~mL} / \mathrm{min}, 254 \mathrm{~nm}$ ) indicated 95\% ee: $\mathrm{t}_{\mathrm{R}}($ minor $)=6.8 \mathrm{~min}, \mathrm{t}_{\mathrm{R}}($ major $)=16.2 \mathrm{~min}$.

$\mathrm{mV}$

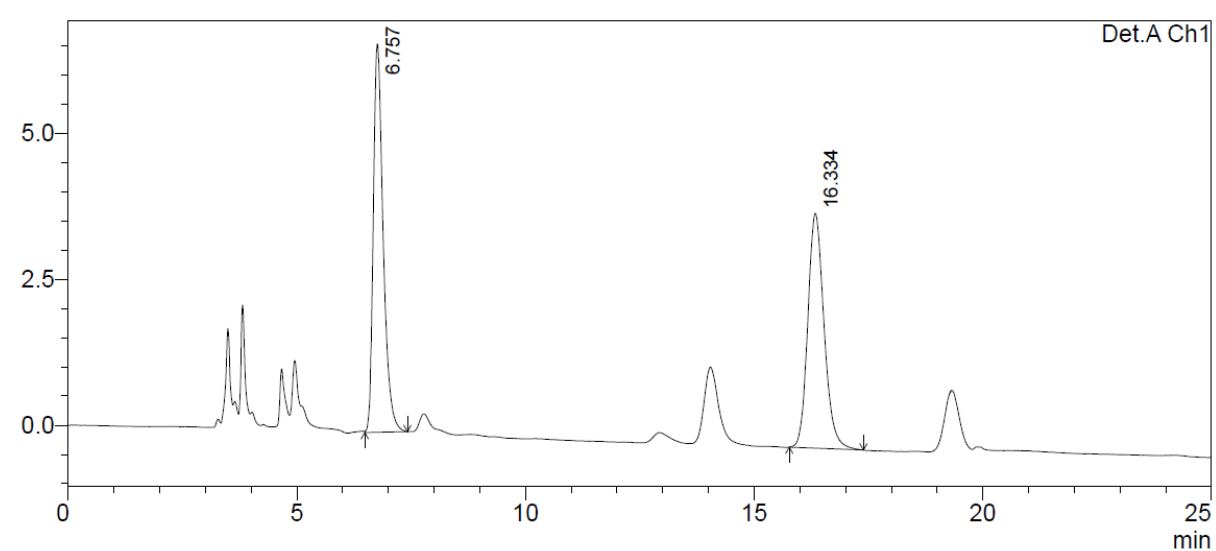

1 Det.A Ch1/254nm

Detector A Ch1 254nm
\begin{tabular}{|r|r|r|r|r|r|}
\hline Peak\# & Ret. Time & \multicolumn{1}{c|}{ Area } & Height & Area $\%$ & Height $\%$ \\
\hline 1 & 6.757 & 99989 & 6656 & 50.138 & 62.331 \\
\hline 2 & 16.334 & 99436 & 4022 & 49.862 & 37.669 \\
\hline Total & & 199425 & 10678 & 100.000 & 100.000 \\
\hline
\end{tabular}

$\mathrm{mV}$

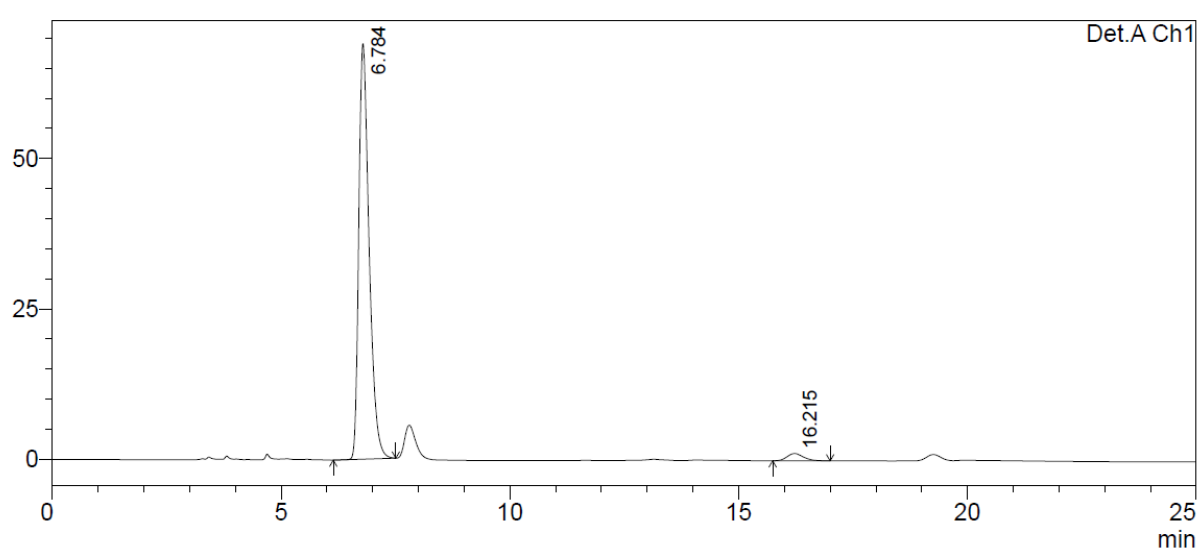

1 Det.A Ch1/254nm

PeakTable

Detector A Ch1 $254 \mathrm{~nm}$
\begin{tabular}{|r|r|r|r|r|r|}
\hline Peak\# & Ret. Time & \multicolumn{1}{|c|}{ Area } & \multicolumn{1}{c|}{ Height } & Area $\%$ & Height $\%$ \\
\hline 1 & 6.784 & 1085107 & 69035 & 97.304 & 98.284 \\
\hline 2 & 16.215 & 30061 & 1205 & 2.696 & 1.716 \\
\hline Total & & 1115168 & 70240 & 100.000 & 100.000 \\
\hline
\end{tabular}




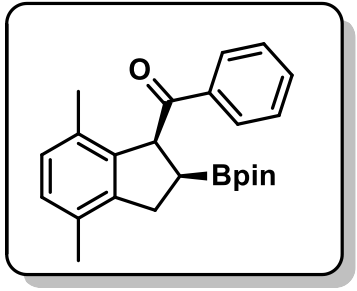

((1S,2S)-4,7-dimethyl-2-(4,4,5,5-tetramethyl-1,3,2-dioxaborolan-2-yl)2,3-dihydro-1H-inden-1-yl)(phenyl)methanone (3v) HPLC analysis (IF, Hexane/IPA $=99 / 1,1 \mathrm{~mL} / \mathrm{min}, 230 \mathrm{~nm})$ indicated $94 \%$ ee: $\mathrm{t}_{\mathrm{R}}($ major $)=$ $12.3 \min , \mathrm{t}_{\mathrm{R}}($ minor $)=17.7 \mathrm{~min}$.

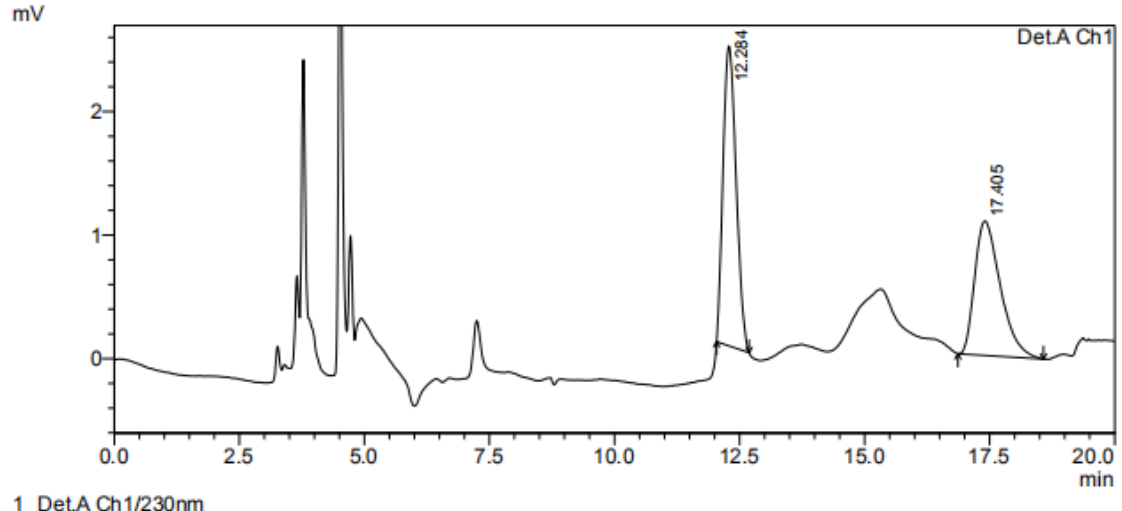

1 Det.A Ch1/230nm

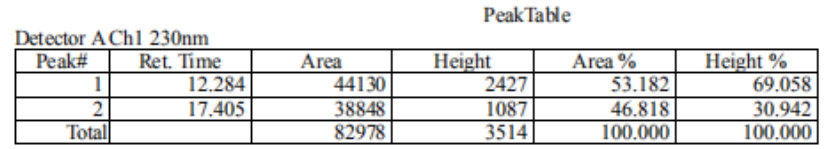

$\mathrm{mV}$

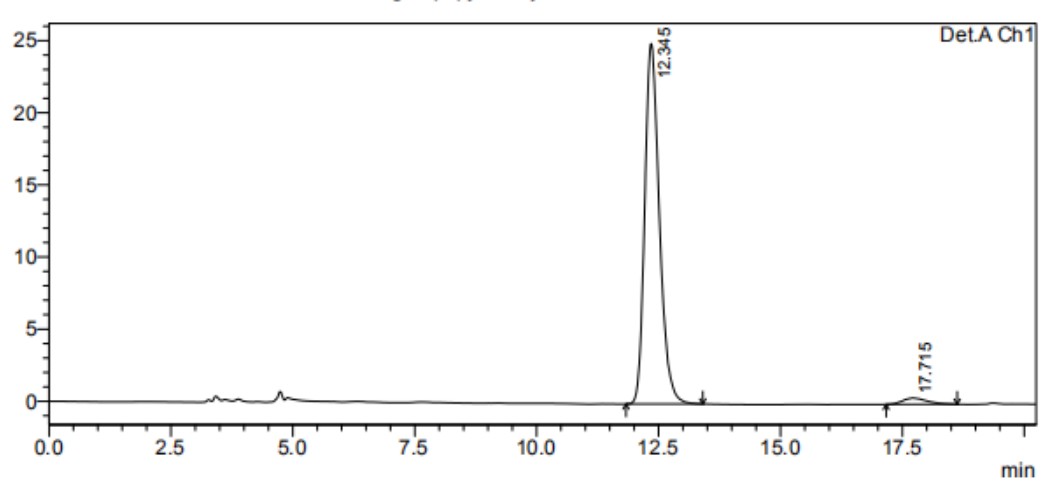

1 Det.A Ch $1 / 230 \mathrm{~nm}$

Detector A Ch1 230nm
\begin{tabular}{r|r|r|r|r|r|}
\hline Peak\# & Ret. Time & \multicolumn{1}{c|}{ Area } & \multicolumn{1}{c|}{ Height } & Area $\%$ & Height $\%$ \\
\hline 1 & 12.345 & 522512 & 24982 & 97.203 & 98.318 \\
\hline 2 & 17.715 & 15034 & 427 & 2.797 & 1.682 \\
\hline Total & & 537545 & 25410 & 100.000 & 100.000 \\
\hline
\end{tabular}




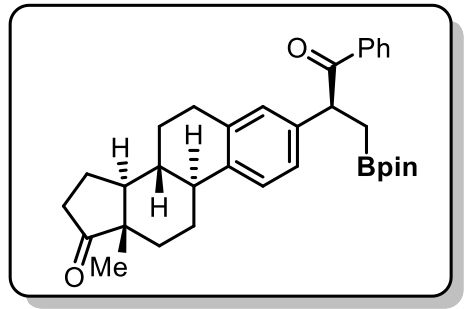

$(8 R, 9 S, 13 S, 14 S)-13-$ methyl-3-((S)-1-oxo-1-phenyl-3-(4,4,5,5-tetr amethyl-1,3,2-dioxaborolan-2-yl)propan-2-yl)-6,7,8,9,11,12,13,1

4,15,16-decahydro-17H-cyclopenta[a]phenanthren-17-one (3w): $[\alpha]_{\mathrm{D}}{ }^{24}=+6.5,\left(\mathrm{c}=0.2, \mathrm{CH}_{2} \mathrm{Cl}_{2}\right)$. HPLC analysis $(\mathrm{IF}$, Hexane/IPA $=$ $99 / 1,1 \mathrm{~mL} / \mathrm{min}, 254 \mathrm{~nm})$ indicated $73 \%$ ee: $\mathrm{t}_{\mathrm{R}}($ major $)=16.8 \mathrm{~min}$, $t_{R}($ minor $)=25.0 \mathrm{~min}$.

$\mathrm{mV}$

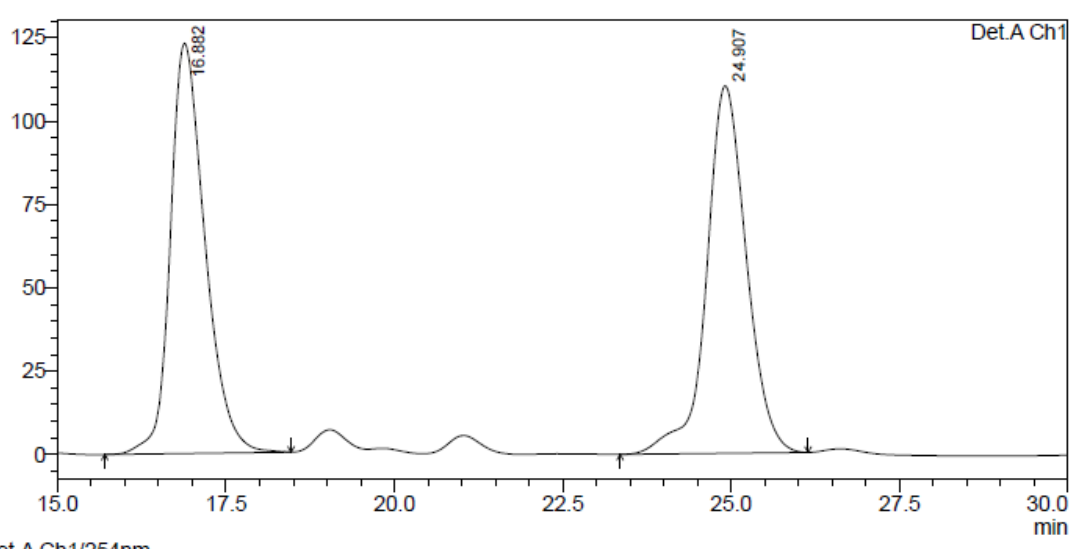

1 Det.A Ch1/254nm

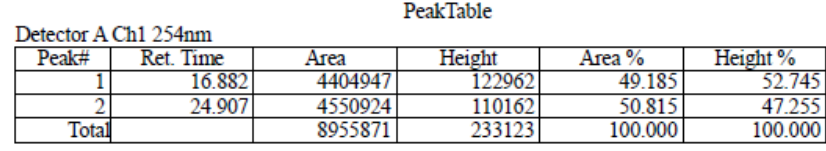

$\mathrm{mV}$

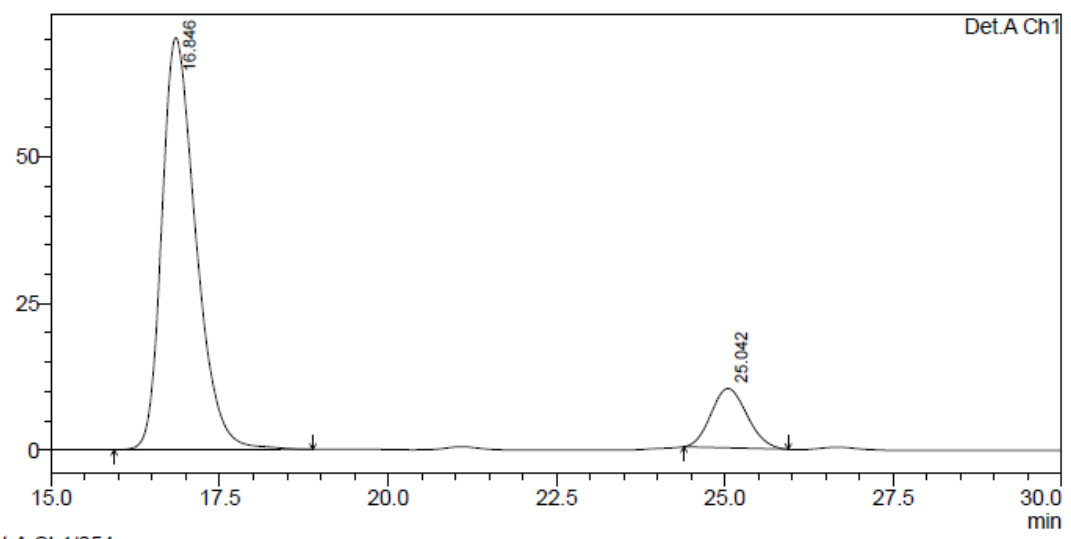

1 Det.A Ch $1 / 254 \mathrm{~nm}$

Detector A Ch1 $254 \mathrm{~nm}$
\begin{tabular}{|r|r|r|r|r|r|}
\hline \multicolumn{1}{|c|}{ Peak\# } & Ret. Time & \multicolumn{1}{c|}{ Area } & Height & Area $\%$ & \multicolumn{1}{c|}{ Height \% } \\
\hline 1 & 16.846 & 2467530 & 70197 & 86.743 & 87.427 \\
\hline 2 & 25.042 & 377105 & 10095 & 13.257 & 12.573 \\
\hline Total & & 2844635 & 80292 & 100.000 & 100.000 \\
\hline
\end{tabular}




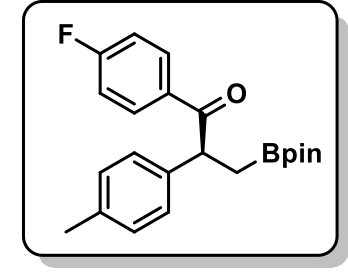

(S)-1-(4-fluorophenyl)-3-(4,4,5,5-tetramethyl-1,3,2-dioxaborolan-2-yl)-2-

(p-tolyl)propan-1-one $(4 a):[\alpha]_{\mathrm{D}}{ }^{24}=+99.8,\left(\mathrm{c}=0.2, \mathrm{CH}_{2} \mathrm{Cl}_{2}\right)$. HPLC analysis (IF, Hexane/IPA = 99/1, $1 \mathrm{~mL} / \mathrm{min}, 254 \mathrm{~nm}$ ) indicated 92\% ee: $\mathrm{t}_{\mathrm{R}}$ $($ major $)=14.3 \min , t_{R}($ minor $)=18.3 \min$.

$\mathrm{mV}$

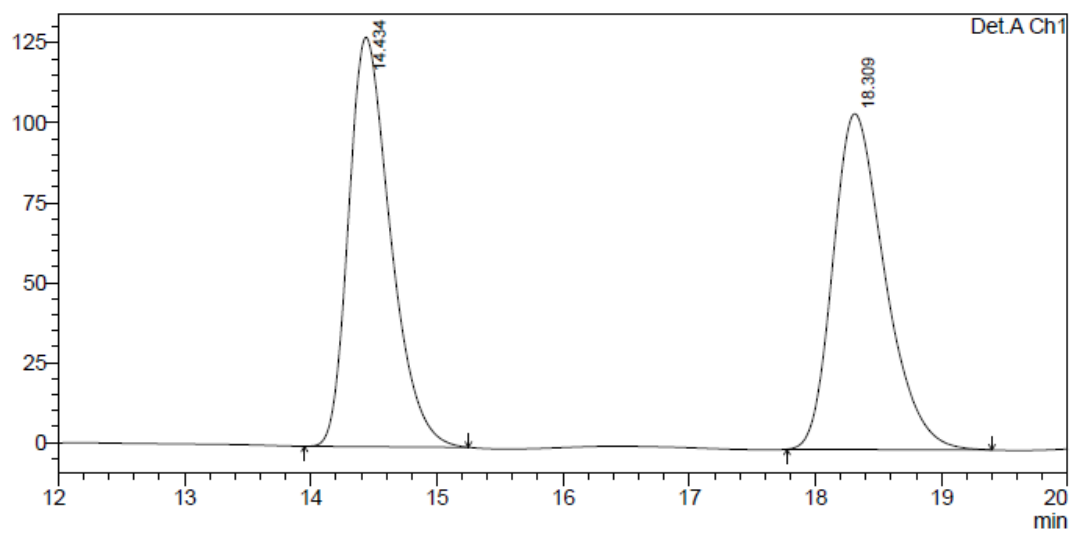

1. Det.A Ch $1 / 254 \mathrm{~nm}$

PeakTable

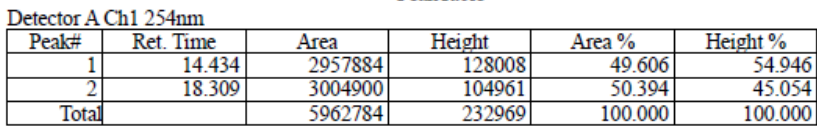

$\mathrm{mV}$

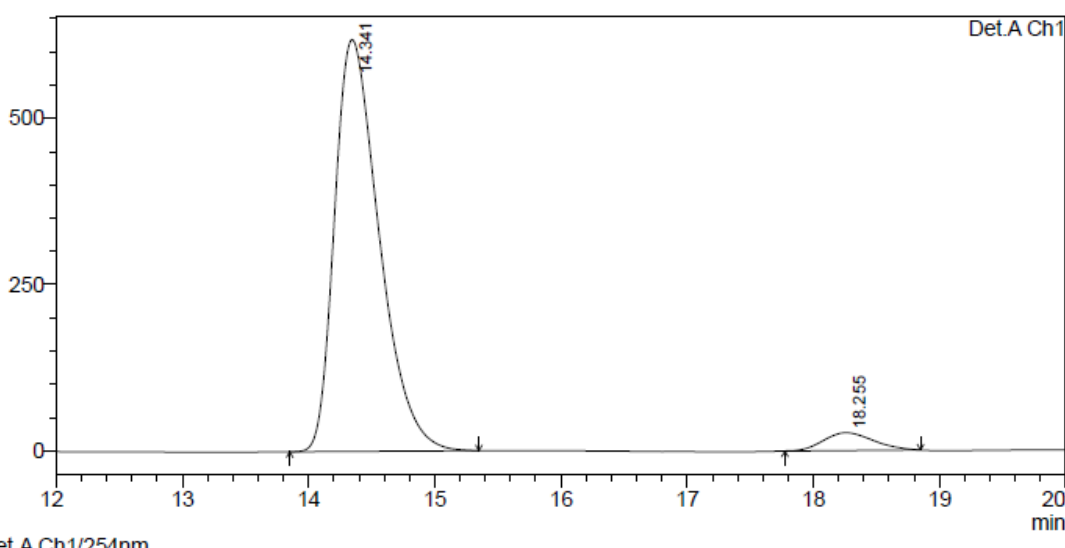

1 Det.A Ch $1 / 254 \mathrm{~nm}$

Detector A Ch1 254nm
\begin{tabular}{|r|r|r|r|r|r|}
\hline Peak\# & Ret. Time & \multicolumn{1}{|c|}{ Area } & Height & Area $\%$ & Height $\%$ \\
\hline 1 & 14.341 & 15256653 & 619430 & 95.355 & 95.825 \\
\hline 2 & 18.255 & 743238 & 26987 & 4.645 & 4.175 \\
\hline Total & & 15999891 & 646417 & 100.000 & 100.000 \\
\hline
\end{tabular}




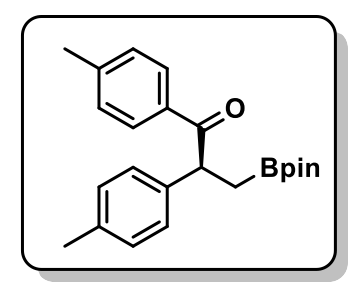

(S)-3-(4,4,5,5-tetramethyl-1,3,2-dioxaborolan-2-yl)-1,2-di-p-tolylpropan-

1-one (4b): $[\alpha]_{\mathrm{D}}^{24}=+71.1,\left(\mathrm{c}=0.2, \mathrm{CH}_{2} \mathrm{Cl}_{2}\right)$. HPLC analysis (IF, Hexane $/ \mathrm{IPA}=99 / 1,1 \mathrm{~mL} / \mathrm{min}, 254 \mathrm{~nm})$ indicated $98 \%$ ee: $\mathrm{t}_{\mathrm{R}}($ major $)=12.5$ $\min , t_{R}(\operatorname{minor})=19.7 \min$.

$\mathrm{mV}$

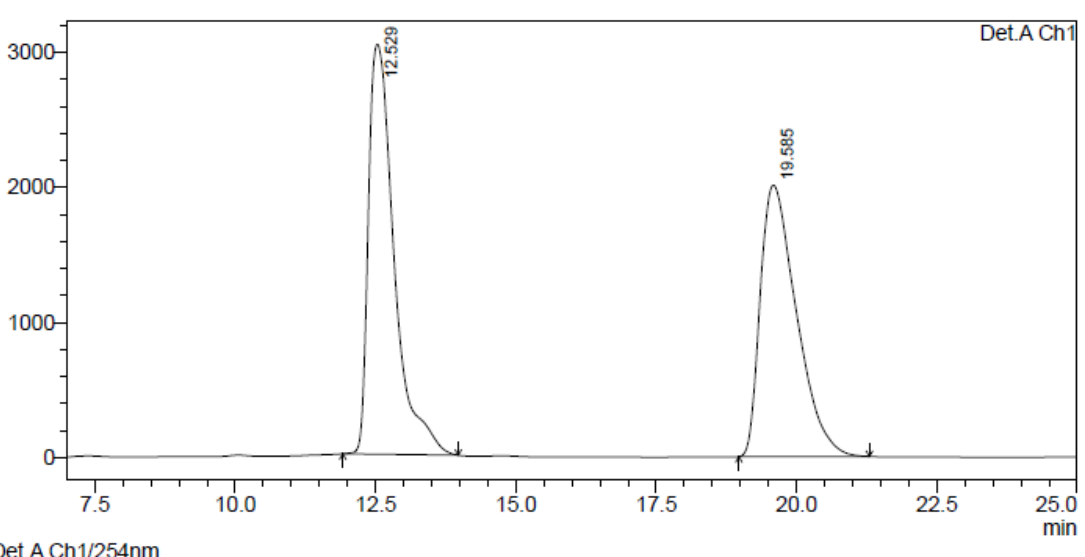

1 Det.A Ch $1 / 254 \mathrm{~nm}$
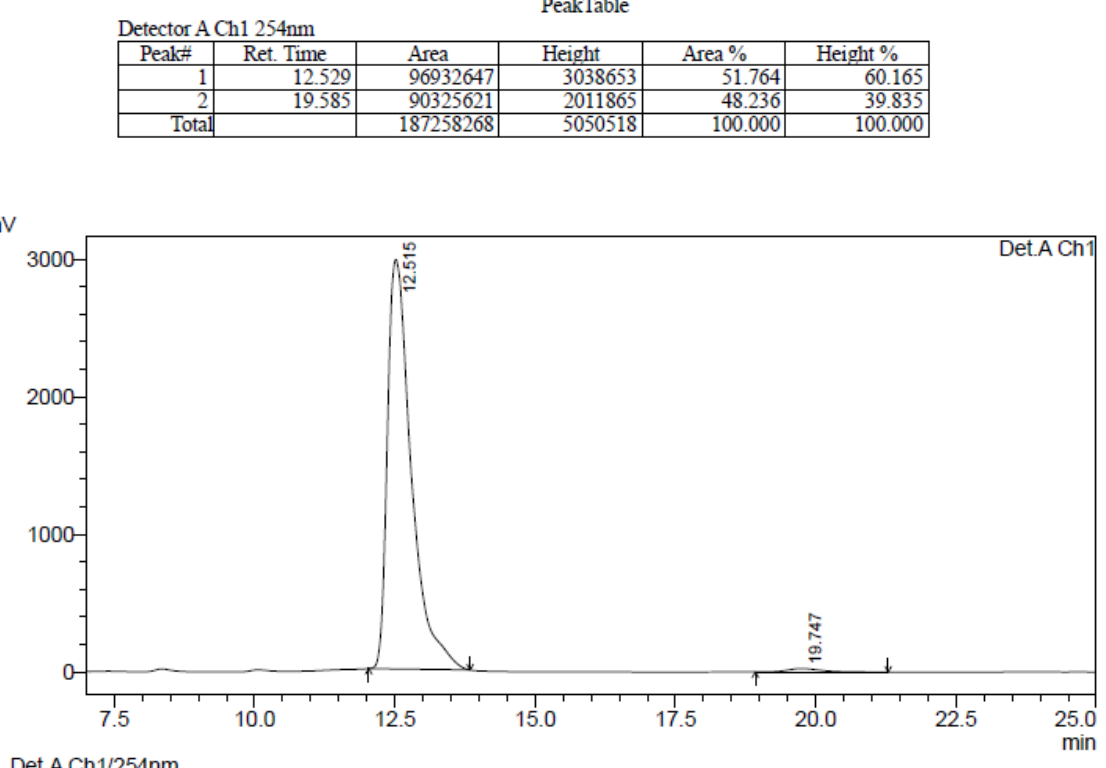

PeakTable

Detector A Ch1 $254 \mathrm{~nm}$
\begin{tabular}{|r|r|r|r|r|r|}
\hline Peak\# & Ret. Time & \multicolumn{1}{|c|}{ Area } & Height & Area $\%$ & Height $\%$ \\
\hline 1 & 12.515 & 88155726 & 2976683 & 98.866 & 99.156 \\
\hline 2 & 19.747 & 1010821 & 25326 & 1.134 & 0.844 \\
\hline Total & & 89166547 & 3002009 & 100.000 & 100.000 \\
\hline
\end{tabular}




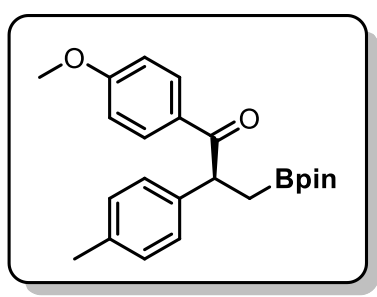

(S)-1-(4-methoxyphenyl)-3-(4,4,5,5-tetramethyl-1,3,2-dioxaborolan-2-

yl)-2-(p-tolyl)propan-1-one $(4 \mathrm{c}):[\alpha]_{\mathrm{D}}{ }^{24}=+120.6,\left(\mathrm{c}=0.2, \mathrm{CH}_{2} \mathrm{Cl}_{2}\right)$.

HPLC analysis (IF, Hexane/IPA = 99/1, $1 \mathrm{~mL} / \mathrm{min}, 254 \mathrm{~nm}$ ) indicated 99\% ee: $\mathrm{t}_{\mathrm{R}}($ major $)=11.2 \mathrm{~min}, \mathrm{t}_{\mathrm{R}}($ minor $)=14.6 \mathrm{~min}$

$\mathrm{m}$

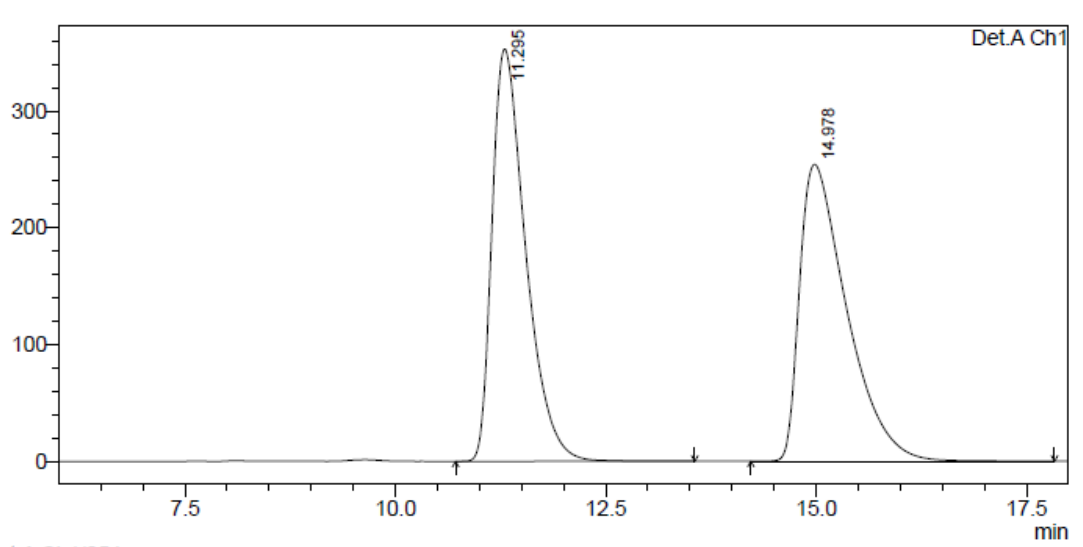

1 Det.A Ch1/254nm

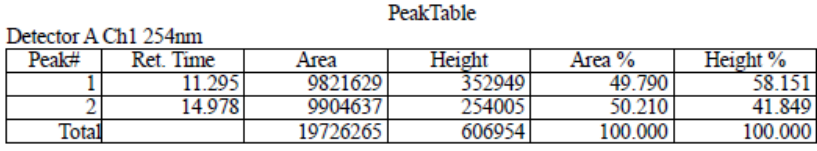

$\mathrm{mV}$

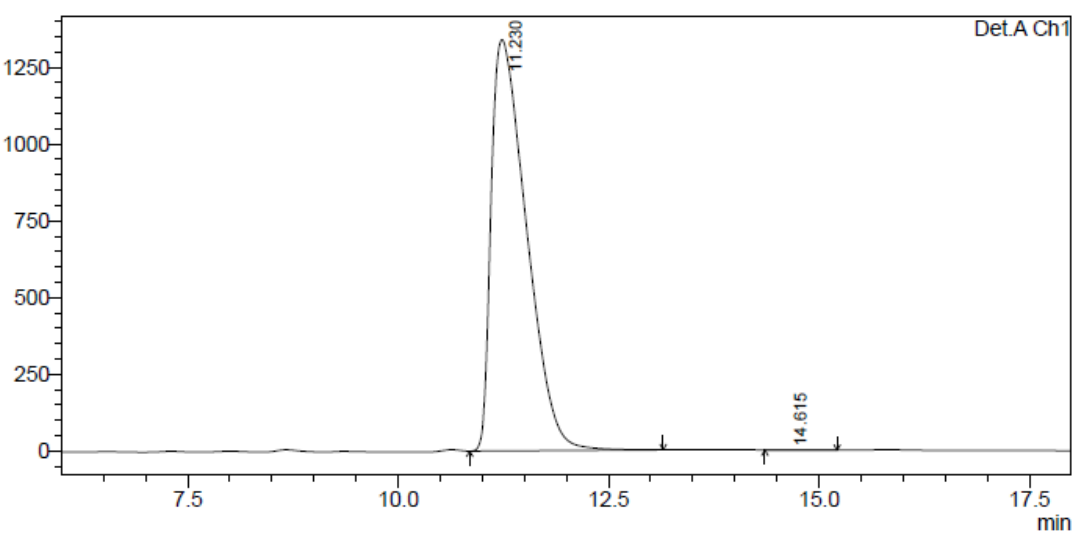

1 Det.A Ch1/254nm

PeakTable

Detector A Ch1 $254 \mathrm{~nm}$
\begin{tabular}{|r|r|r|r|r|r|}
\hline Peak\# & Ret. Time & \multicolumn{1}{|c|}{ Area } & Height & Area \% & Height \% \\
\hline 1 & 11.230 & 39091000 & 1339327 & 99.955 & 99.944 \\
\hline 2 & 14.615 & 17749 & 748 & 0.045 & 0.056 \\
\hline Total & & 39108749 & 1340075 & 100.000 & 100.000 \\
\hline
\end{tabular}




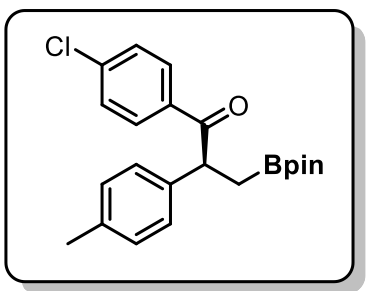

$\mathrm{mV}$

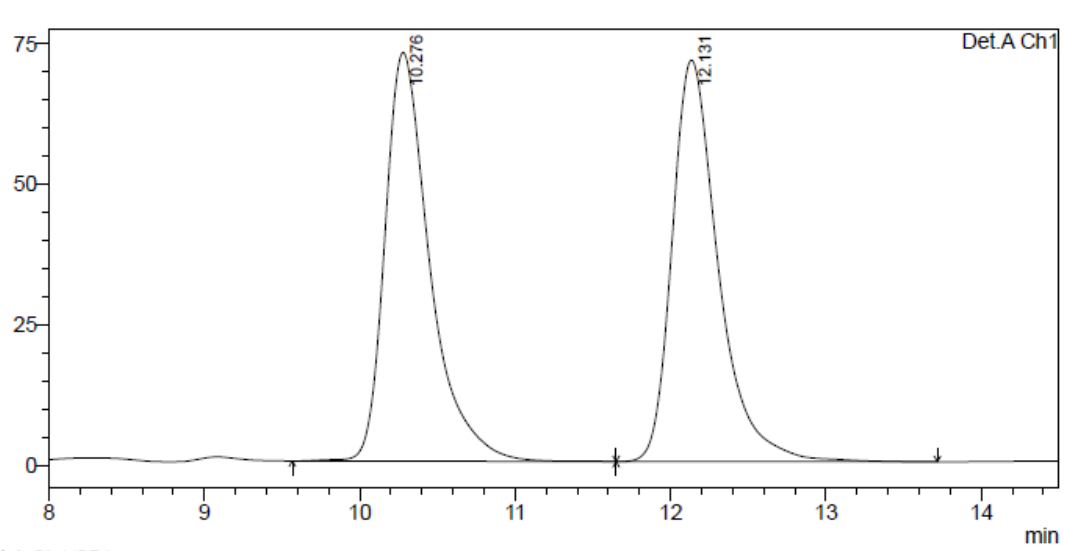

1 Det.A Ch1/254nm

(S)-1-(4-chlorophenyl)-3-(4,4,5,5-tetramethyl-1,3,2-dioxaborolan-2-yl)

-2-(p-tolyl)propan-1-one $(4 d):[\alpha]_{D}^{24}=+120.6,\left(c=0.2, \mathrm{CH}_{2} \mathrm{Cl}_{2}\right)$. HPLC analysis (OD-H, Hexane/IPA = 99/1, $1 \mathrm{~mL} / \mathrm{min}, 254 \mathrm{~nm})$ indicated $96 \%$ ee: $\mathrm{t}_{\mathrm{R}}($ minor $)=10.2 \mathrm{~min}, \mathrm{t}_{\mathrm{R}}($ major $)=12.2 \mathrm{~min}$.

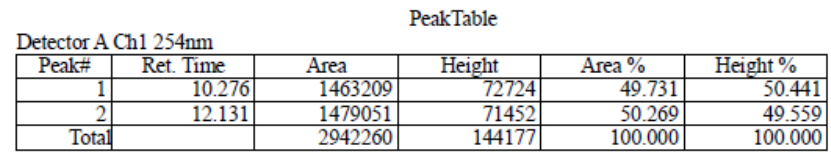

$\mathrm{mV}$

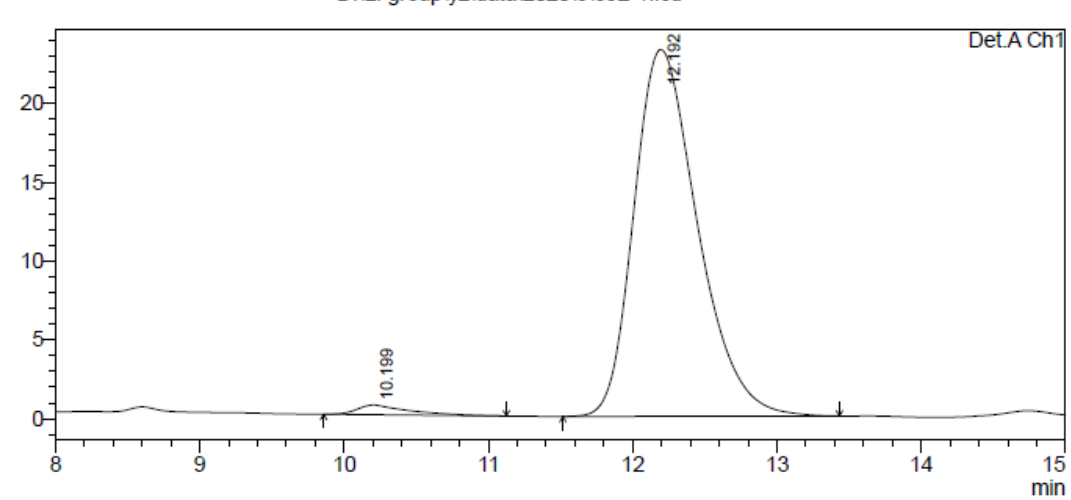

1 Det.A Ch $1 / 254 \mathrm{~nm}$

PeakTable

Detector A Ch1 $254 \mathrm{~nm}$
\begin{tabular}{|r|r|r|r|r|r|}
\hline \multicolumn{1}{|c|}{ Peak\# } & Ret. Time & Area & \multicolumn{1}{c|}{ Height } & Area $\%$ & \multicolumn{1}{c|}{ Height $\%$} \\
\hline 1 & 10.199 & 15173 & 617 & 2.083 & 2.584 \\
\hline 2 & 12.192 & 713144 & 23256 & 97.917 & 97.416 \\
\hline Total & & 728316 & 23873 & 100.000 & 100.000 \\
\hline
\end{tabular}




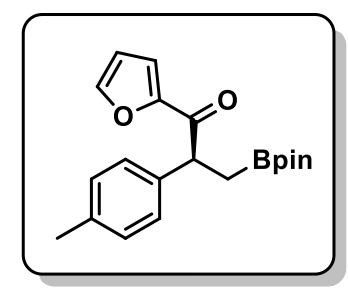

(S)-1-(furan-2-yl)-3-(4,4,5,5-tetramethyl-1,3,2-dioxaborolan-2-yl)-2-(p-to

lyl)propan-1-one (4e): $[\alpha]_{D^{24}}=+39.3,\left(\mathrm{c}=0.2, \mathrm{CH}_{2} \mathrm{Cl}_{2}\right)$. HPLC analysis (OD-H, Hexane/IPA $=99 / 1,0.8 \mathrm{~mL} / \mathrm{min}, 254 \mathrm{~nm})$ indicated $98 \%$ ee: $\mathrm{t}_{\mathrm{R}}$ $(\operatorname{minor})=23.5 \mathrm{~min}, \mathrm{t}_{\mathrm{R}}($ major $)=24.9 \mathrm{~min}$

$\mathrm{mV}$

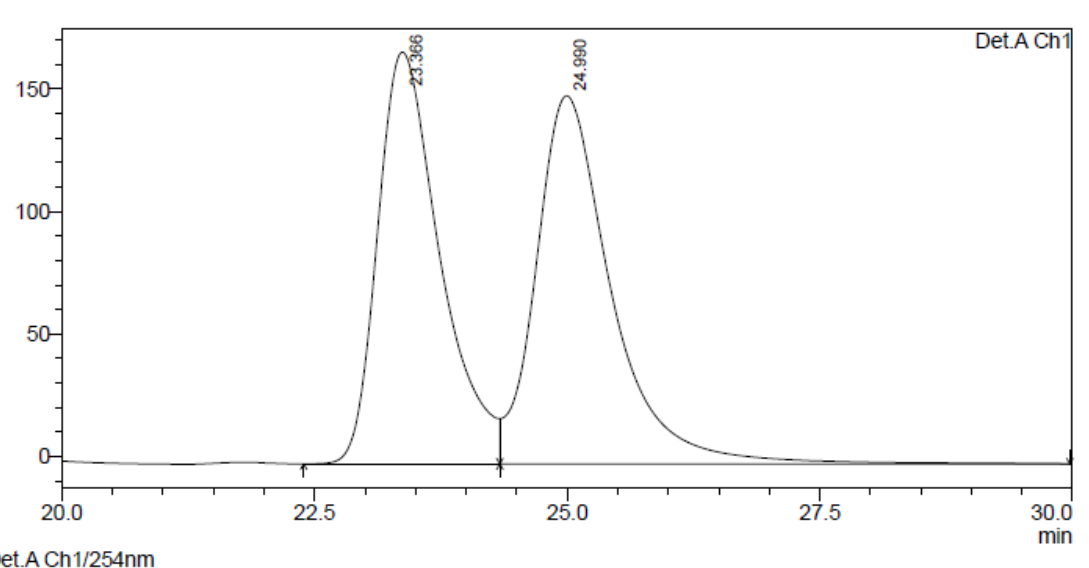

1 Det.A Ch1/254nm

PeakTable

Detector A Ch1 $254 \mathrm{~nm}$
\begin{tabular}{|r|r|r|r|r|r|}
\hline Peak\#\# & Ret. Time & \multicolumn{1}{c|}{ Area } & Height & Area $\%$ & \multicolumn{1}{c|}{ Height $\%$} \\
\hline 1 & 23.366 & 7205550 & 168162 & 48.011 & 52.802 \\
\hline 2 & 24.990 & 7802660 & 150312 & 51.989 & 47.198 \\
\hline Total & & 15008210 & 318474 & 100.000 & 100.000 \\
\hline
\end{tabular}

$\mathrm{mV}$

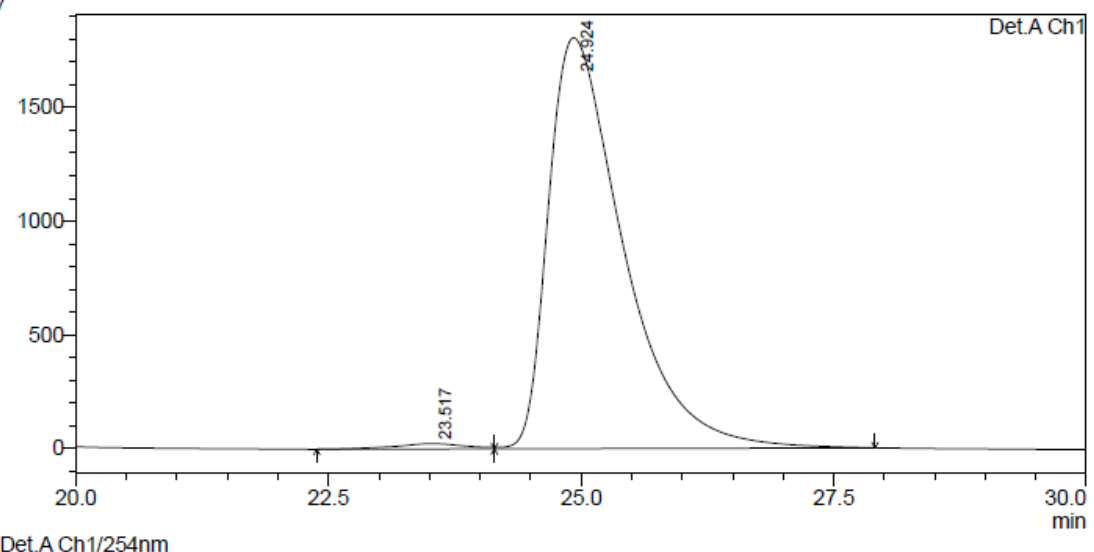

PeakTable

Detector A Ch1 $254 \mathrm{~nm}$
\begin{tabular}{|r|r|r|r|r|r|}
\hline Peak\# & Ret. Time & \multicolumn{1}{c|}{ Area } & Height & Area $\%$ & Height $\%$ \\
\hline 1 & 23.517 & 1141896 & 23640 & 1.209 & 1.291 \\
\hline 2 & 24.924 & 93333300 & 1807062 & 98.791 & 98.709 \\
\hline Total & & 94475196 & 1830703 & 100.000 & 100.000 \\
\hline
\end{tabular}




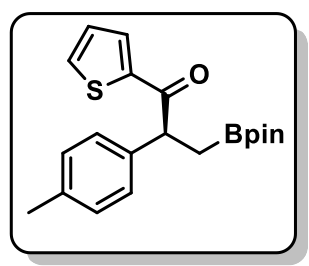

(S)-3-(4,4,5,5-tetramethyl-1,3,2-dioxaborolan-2-yl)-1-(thiophen-2-yl)-2-(p-t

olyl)propan-1-one (4f): $[\alpha]_{\mathrm{D}}{ }^{24}=+15.5,\left(\mathrm{c}=0.2, \mathrm{CH}_{2} \mathrm{Cl}_{2}\right)$. HPLC analysis $(\mathrm{OD}-\mathrm{H}$, Hexane $/ \mathrm{IPA}=99 / 1,1 \mathrm{~mL} / \mathrm{min}, 254 \mathrm{~nm})$ indicated $99 \%$ ee: $\mathrm{t}_{\mathrm{R}}($ minor $)=$ $8.3 \min , \mathrm{t}_{\mathrm{R}}($ major $)=9.9 \mathrm{~min}$.
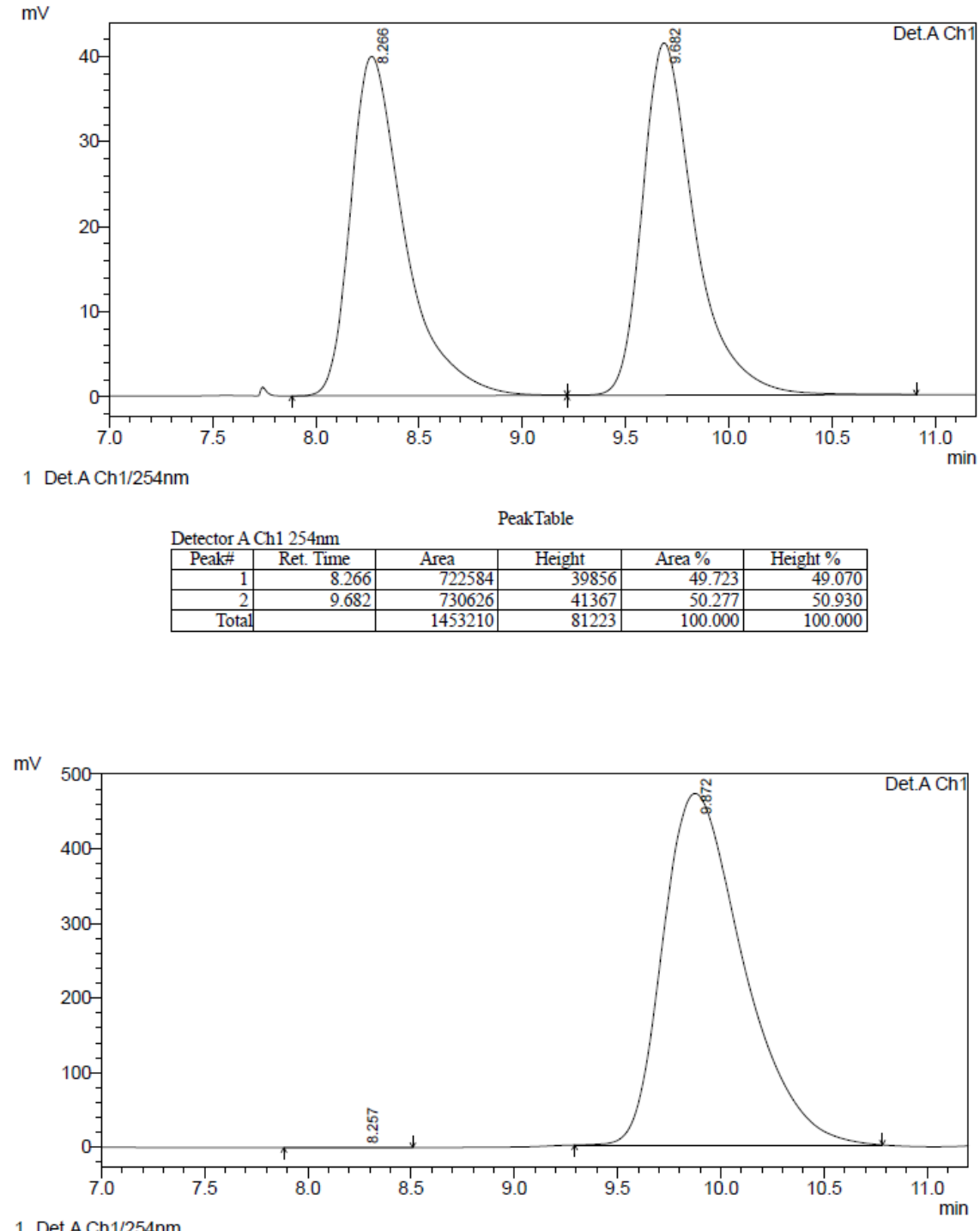

1 Det.A Ch $1 / 254 \mathrm{~nm}$

\begin{tabular}{|c|c|c|c|c|c|}
\hline \multicolumn{6}{|c|}{ Detector $A$ Cb $1254 \mathrm{~nm}$} \\
\hline $\begin{array}{c}\text { Detector A } \\
\text { Peak\# }\end{array}$ & $\frac{11254 \mathrm{~nm}}{\text { Ret Time }}$ & Aren & Heirht & & Height $0 \%$ \\
\hline 1 & 8.257 & $\frac{\text { Area }}{3015}$ & $\frac{{ }^{\text {Height }}}{191}$ & $\frac{\text { Area } \%}{0.024}$ & 0.041 \\
\hline$\frac{1}{2}$ & 9.872 & 12781152 & 470839 & 99.976 & 99.959 \\
\hline Total & & 12784166 & 471030 & 100.000 & 100.000 \\
\hline
\end{tabular}




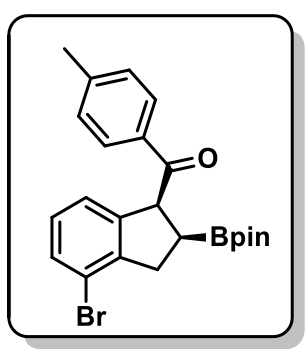

((1S,2S)-4-bromo-2-(4,4,5,5-tetramethyl-1,3,2-dioxaborolan-2-yl)-2,3-dih

ydro-1H-inden-1-yl)(p-tolyl)methanone (4g) HPLC analysis (IF, Hexane/IPA $=99 / 1,1 \mathrm{~mL} / \mathrm{min}, 230 \mathrm{~nm})$ indicated 93\% ee: $\mathrm{t}_{\mathrm{R}}($ minor $)=$ $12.0 \min , \mathrm{t}_{\mathrm{R}}($ major $)=15.6 \min$.

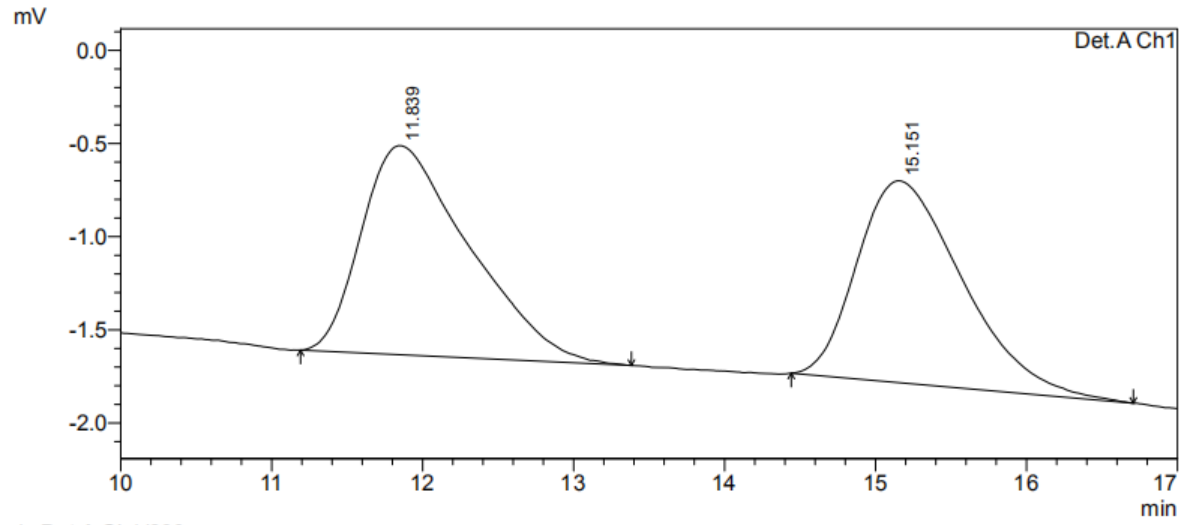

1 Det.A Ch1/230nm

Detector A Ch1 230nm
\begin{tabular}{|r|r|r|r|r|r|}
\hline Peak\# & Ret. Time & Area & Height & Area $\%$ & Height $\%$ \\
\hline 1 & 11.839 & 55631 & 1122 & 51.361 & 50.881 \\
\hline 2 & 15.151 & 52682 & 1083 & 48.639 & 49.119 \\
\hline Total & & 108313 & 2206 & 100.000 & 100.000 \\
\hline
\end{tabular}

$\mathrm{mV}$

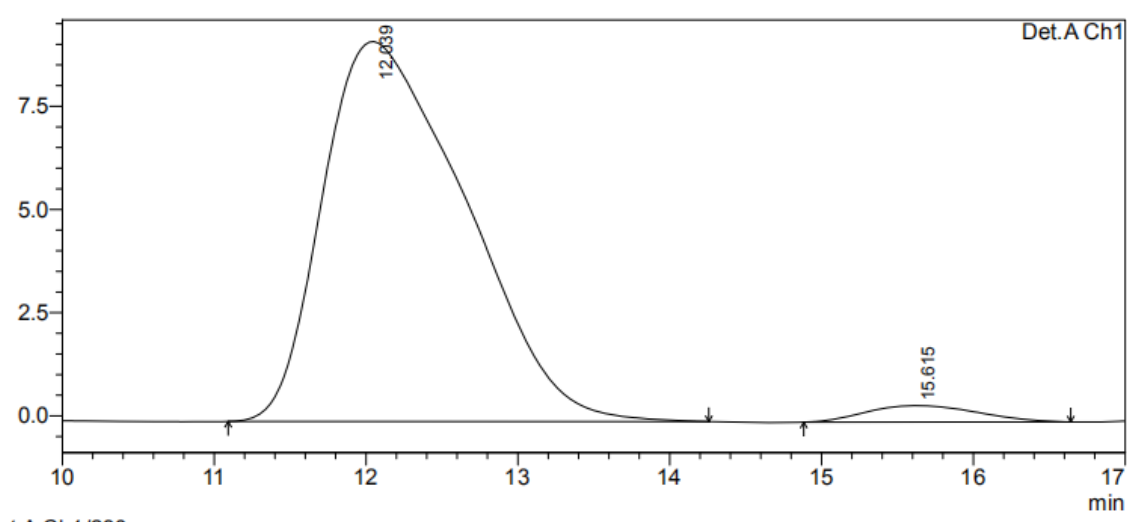

1 Det.A Ch1/230nm

Detector A Ch1 230nm
\begin{tabular}{|r|r|r|r|r|r|}
\hline Peak\# & Ret. Time & \multicolumn{1}{|c|}{ Area } & \multicolumn{1}{c|}{ Height } & Area $\%$ & \multicolumn{1}{c|}{ Height $\%$} \\
\hline 1 & 12.039 & 602362 & 9200 & 96.736 & 95.828 \\
\hline 2 & 15.615 & 20327 & 401 & 3.264 & 4.172 \\
\hline Total & & 622689 & 9600 & 100.000 & 100.000 \\
\hline
\end{tabular}




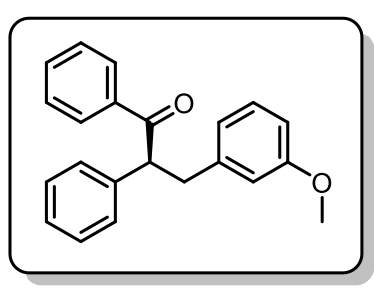

(S)-3-(3-methoxyphenyl)-1,2-diphenylpropan-1-one (5): $[\alpha]_{\mathrm{D}}^{24}=+$

166.2, $\left(\mathrm{c}=0.2, \mathrm{CH}_{2} \mathrm{Cl}_{2}\right)$. HPLC analysis (IF, Hexane/IPA $=98 / 2,1$

$\mathrm{mL} / \mathrm{min}, 254 \mathrm{~nm})$ indicated $87 \%$ ee: $\mathrm{t}_{\mathrm{R}}($ major $)=22.2 \mathrm{~min}, \mathrm{t}_{\mathrm{R}}($ minor $)=$ $31.6 \mathrm{~min}$.

$\mathrm{m} \vee$

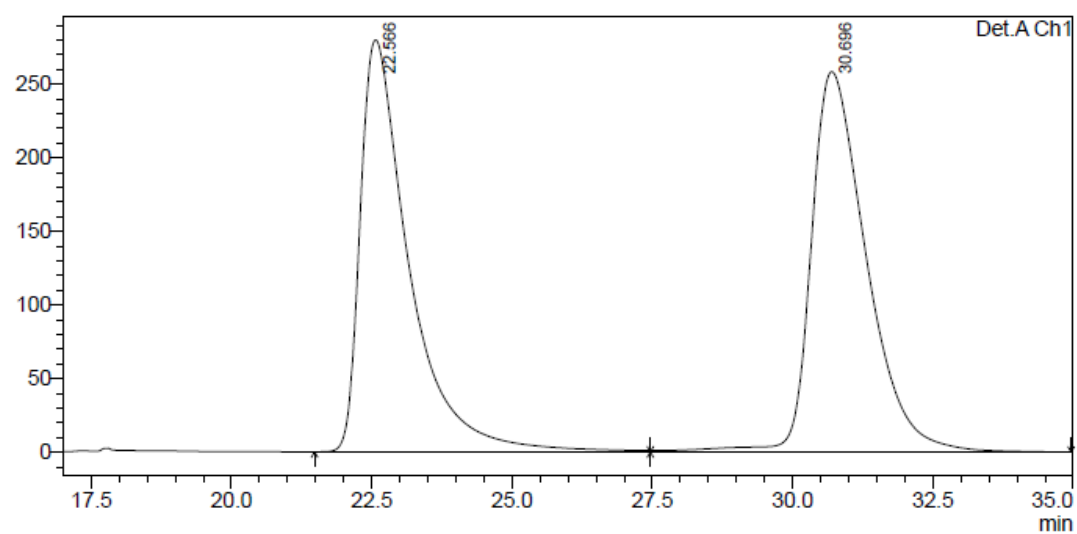

1 Det.A Ch1/254nm

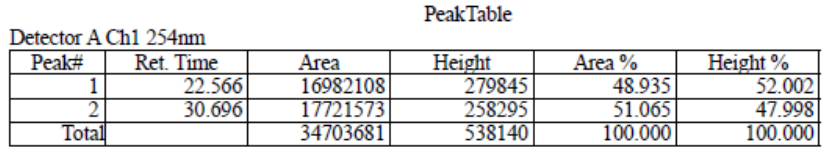

$\mathrm{mV}$

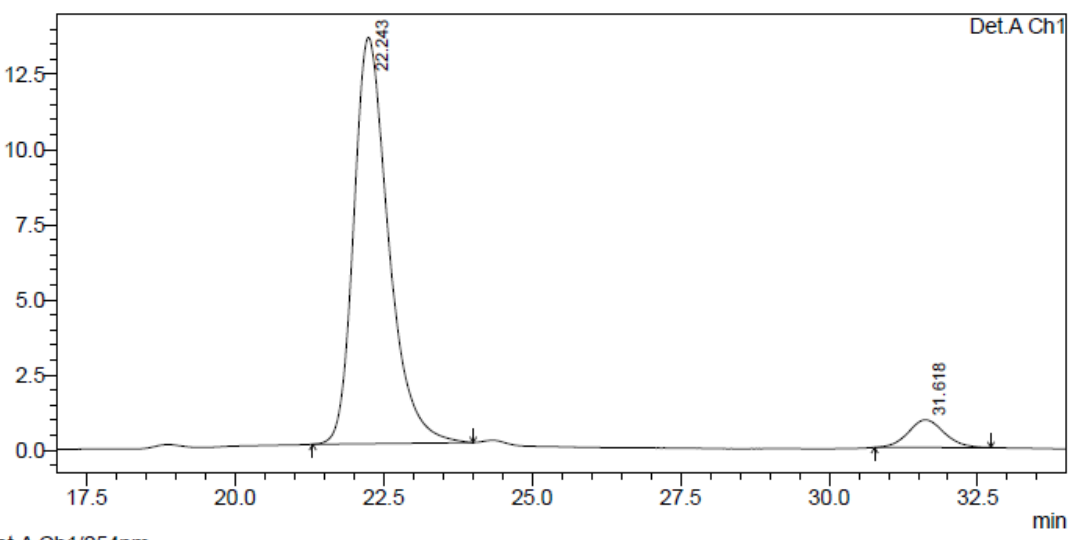

1 Det.A Ch1/254nm

Detector A Ch1 254nm
\begin{tabular}{|r|r|r|r|r|r|}
\hline \multicolumn{1}{|c|}{ Peak\# } & Ret. Time & \multicolumn{1}{c|}{ Area } & Height & Area \% & \multicolumn{1}{c|}{ Height \% } \\
\hline 1 & 22.243 & 550632 & 13509 & 93.423 & 93.624 \\
\hline 2 & 31.618 & 38768 & 920 & 6.577 & 6.376 \\
\hline Total & & 589399 & 14429 & 100.000 & 100.000 \\
\hline
\end{tabular}




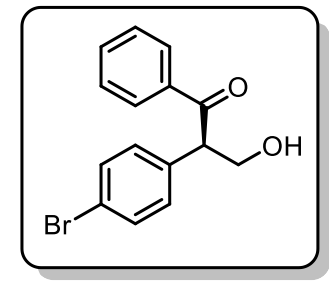

(R)-2-(4-bromophenyl)-3-hydroxy-1-phenylpropan-1-one (6): $[\alpha]_{\mathrm{D}}{ }^{24}=+$ 145.0, $\left(\mathrm{c}=0.2, \mathrm{CH}_{2} \mathrm{Cl}_{2}\right)$. HPLC analysis $(\mathrm{OD}-\mathrm{H}$, Hexane/IPA $=95 / 5,1$ $\mathrm{mL} / \mathrm{min}, 225 \mathrm{~nm})$ indicated $95 \%$ ee: $\mathrm{t}_{\mathrm{R}}($ major $)=38.7 \mathrm{~min}, \mathrm{t}_{\mathrm{R}}($ minor $)=45.8$ min.

$\mathrm{mV}$

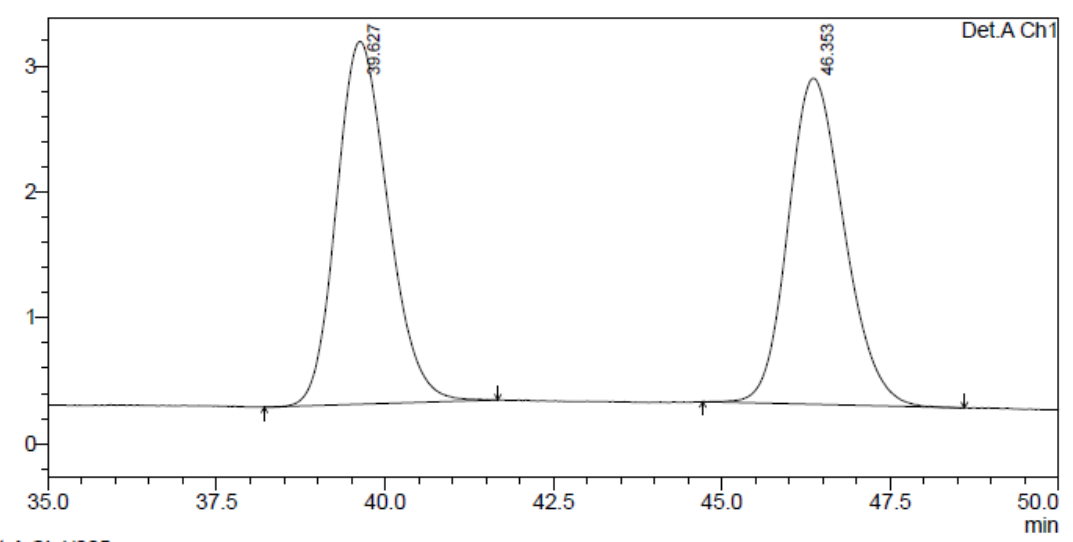

1 Det.A Ch $1 / 225 \mathrm{~nm}$

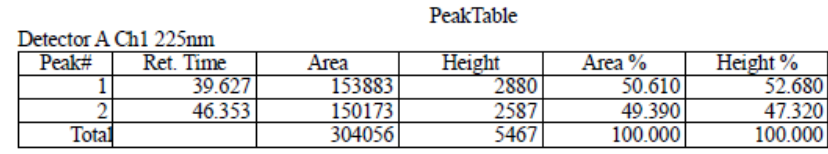

$\mathrm{mV}$

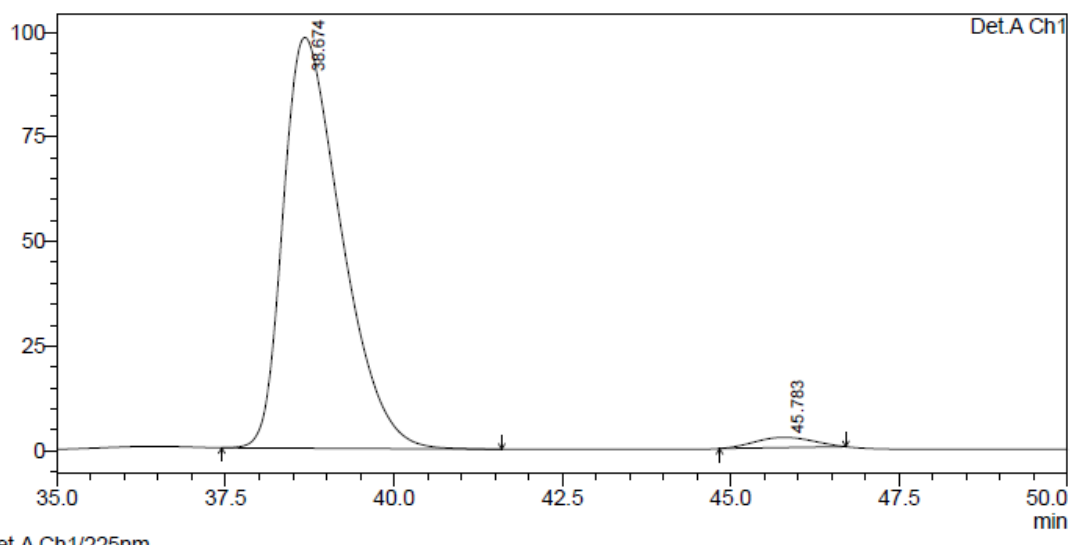

1 Det.A Ch $1 / 225 \mathrm{~nm}$

PeakTable
\begin{tabular}{|r|r|r|r|r|r|} 
Detector A Ch1 225nm \\
\hline Peak\# & Ret. Time & \multicolumn{1}{|c|}{ Area } & \multicolumn{1}{c|}{ Height } & Area \% & \multicolumn{1}{c|}{ Height $\%$} \\
\hline 1 & 38.674 & 5907102 & 98150 & 97.577 & 97.554 \\
\hline 2 & 45.783 & 146669 & 2460 & 2.423 & 2.446 \\
\hline Total & & 6053771 & 100611 & 100.000 & 100.000 \\
\hline
\end{tabular}




\section{Absolute Stereochemistry Determination}

\section{(1) Absolute Stereochemistry Determination.}

The absolute stereochemistry of $\mathbf{3}$ was determined by HPLC spectra comparison of $\mathbf{3 p}$ with $\mathbf{3 p}$ ' obtained by recrystallization of Brown's work. ${ }^{5}$

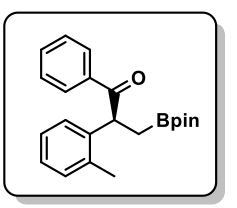

(S)-1-phenyl-3-(4,4,5,5-tetramethyl-1,3,2-dioxaborolan-2-yl)-2-(o-tolyl)propan -1-one (3p): HPLC analysis (IA, Hexane/IPA = 99/1, $1 \mathrm{~mL} / \mathrm{min}, 254 \mathrm{~nm}$ )
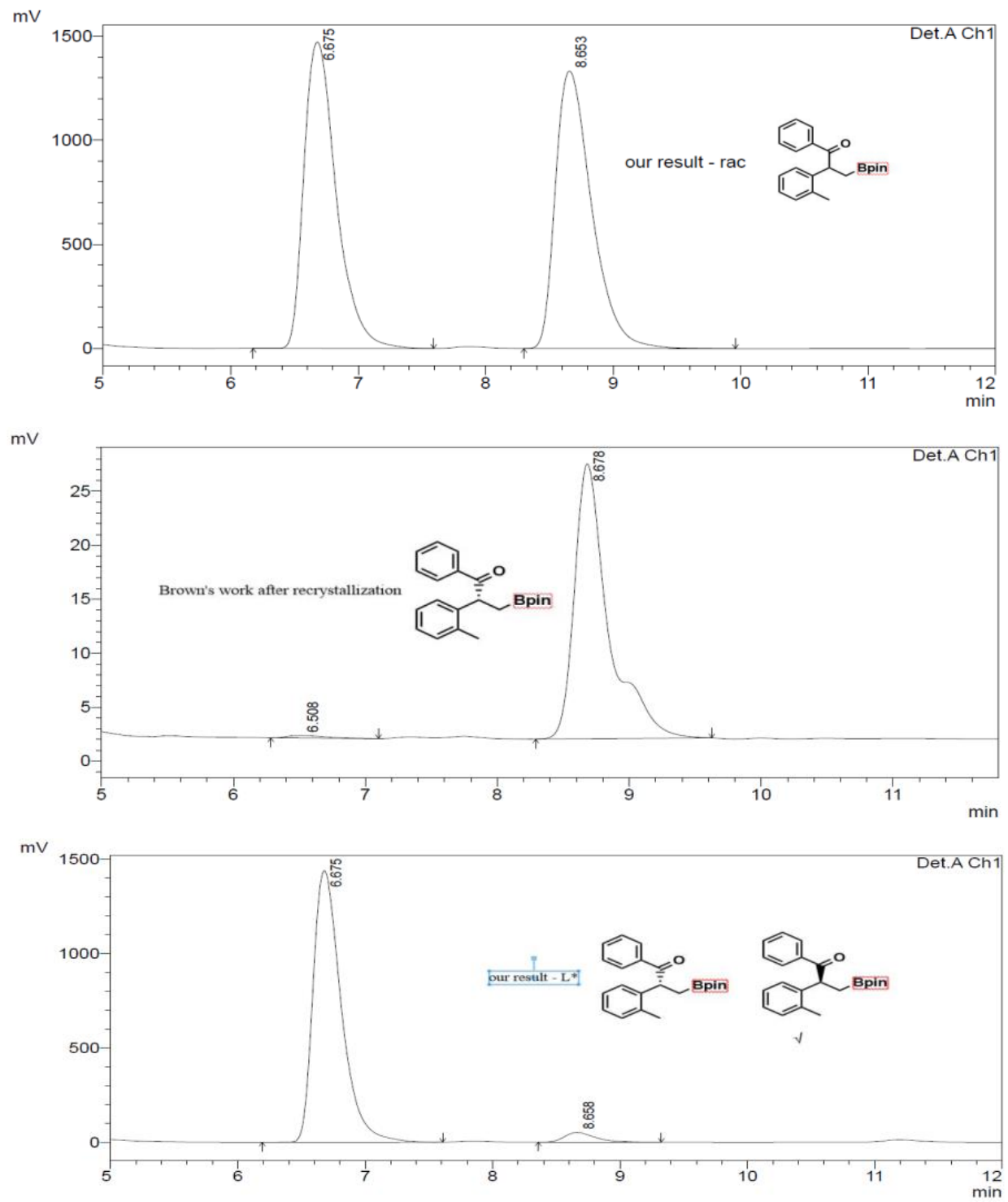

Conclusion: The results above showed that the absolute configuration of $\mathbf{3 p}$ we synthesized was the opposite configuration of Brown's work. 
(2) NOESY experimental

NOESY of $\mathbf{3 v}, 400 \mathrm{MHz}, \mathrm{CDCl}_{3}$<smiles>C[C@]1([Pb]2CCCCC2)c2ccccc2C[C@]1(C(=O)c1ccccc1)c1ccccc1</smiles>

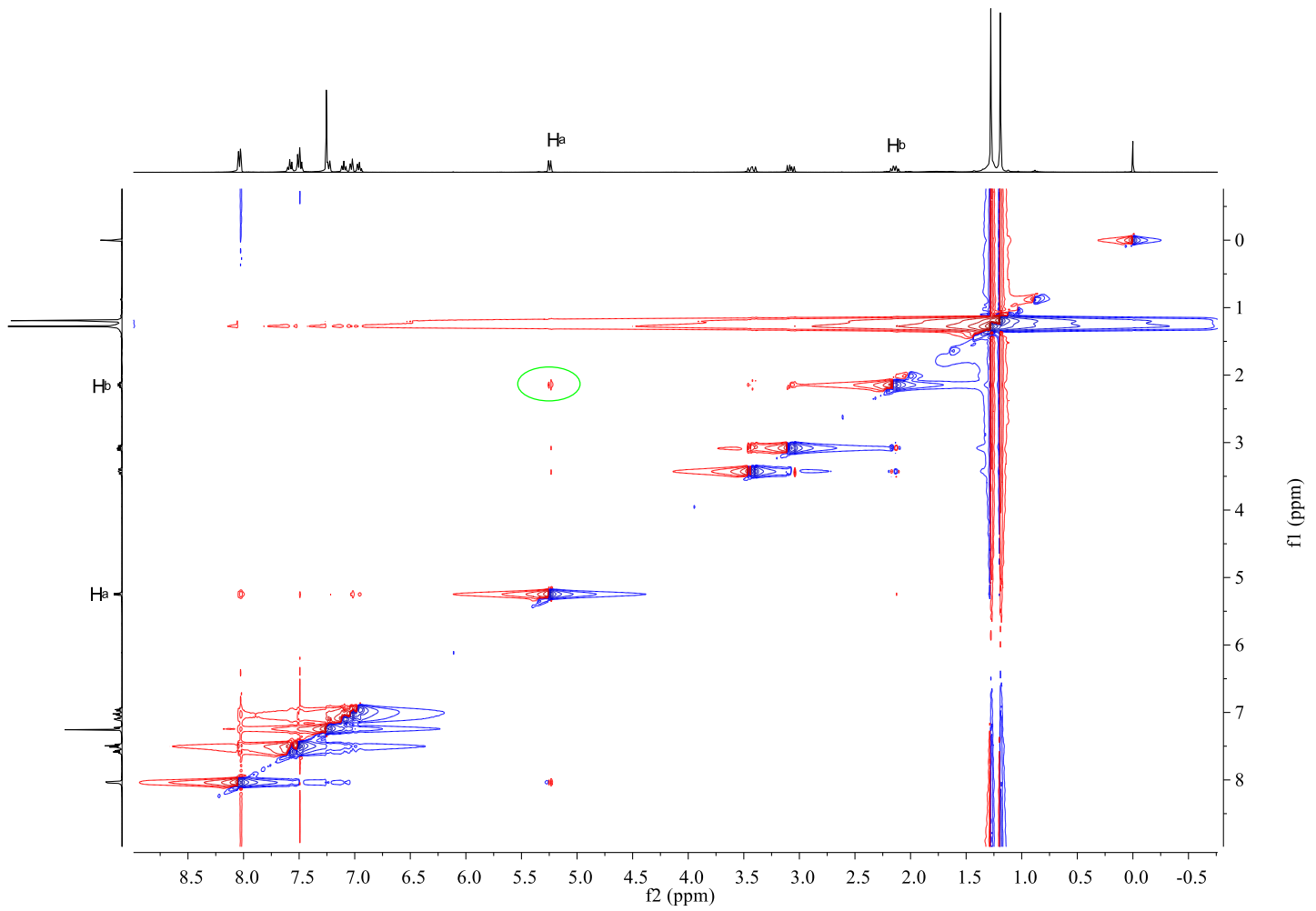




\section{Spectra of New Compounds}

(S)-1,2-diphenyl-3-(4,4,5,5-tetramethyl-1,3,2-dioxaborolan-2-yl)propan-1-one (3a): ${ }^{1} \mathrm{H}$ NMR 400 $\mathrm{MHz},{ }^{13} \mathrm{C}\{1 \mathrm{H}\}$ NMR $101 \mathrm{MHz}, \mathrm{CDCl}_{3}$
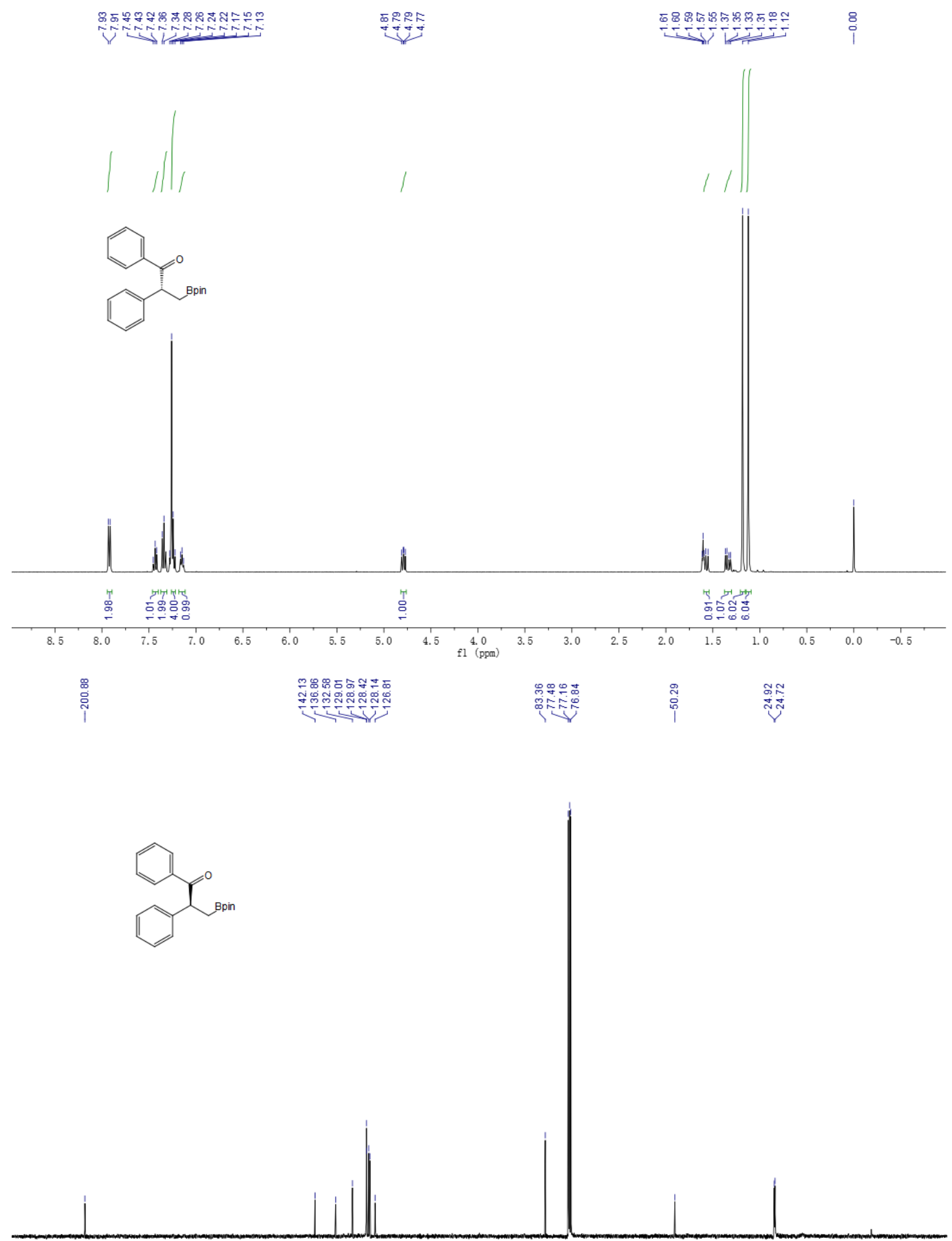

$\begin{array}{llllllllllll}210 & 200 & 190 & 180 & 170 & 160 & 150 & 140 & 130 & 120 & 110 & 100 \\ & & & & & & & & & & & \end{array}$ 
NMR $400 \mathrm{MHz},{ }^{13} \mathrm{C}\{1 \mathrm{H}\} \mathrm{NMR} 101 \mathrm{MHz}, \mathrm{CDCl}_{3}$

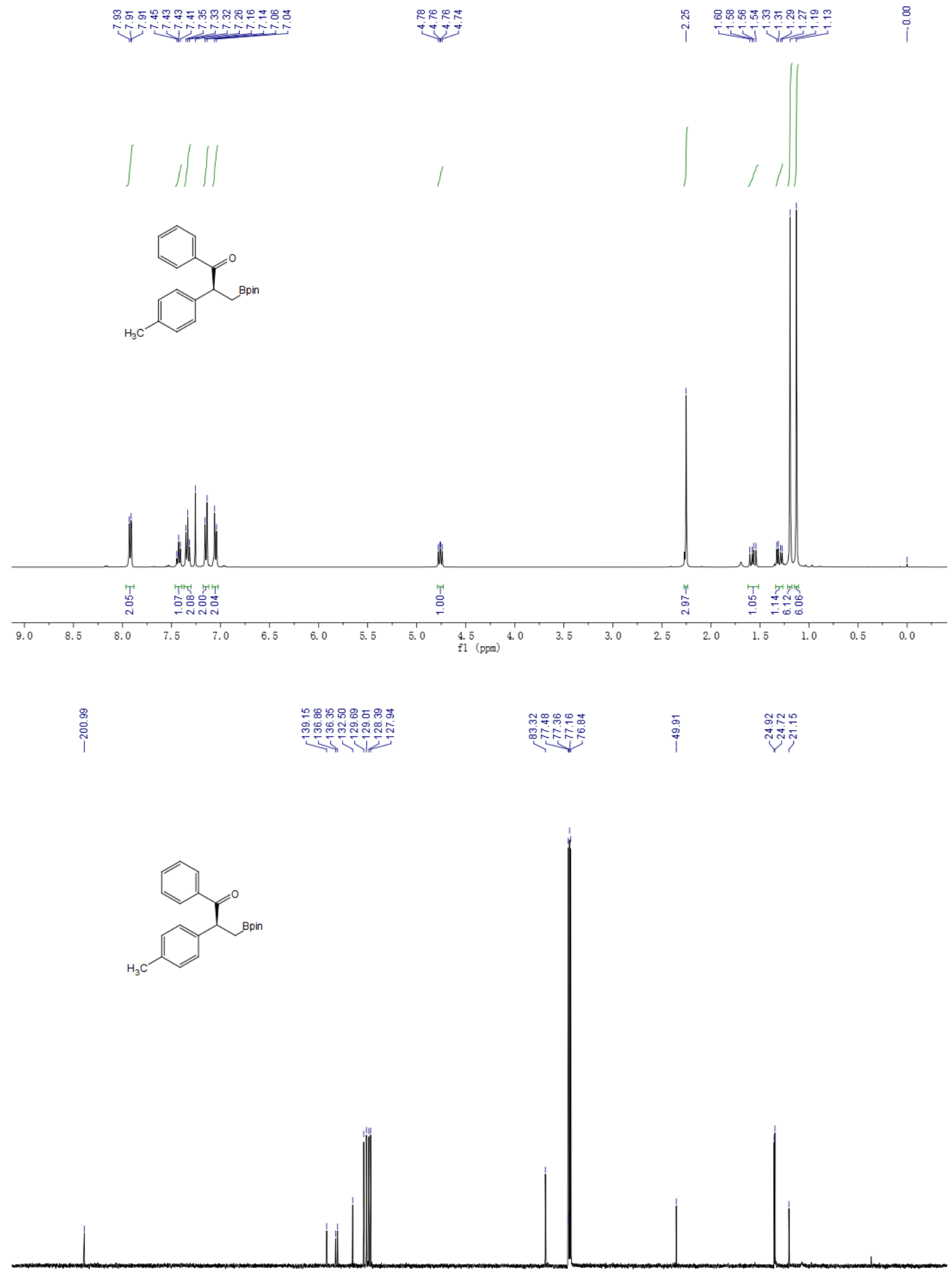

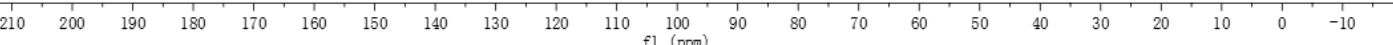


(S)-2-(4-ethylphenyl)-1-phenyl-3-(4,4,5,5-tetramethyl-1,3,2-dioxaborolan-2-yl)propan-1-one (3c): ${ }^{1} \mathrm{H}$ NMR $400 \mathrm{MHz},{ }^{13} \mathrm{C}\{1 \mathrm{H}\}$ NMR $101 \mathrm{MHz}, \mathrm{CDCl}_{3}$
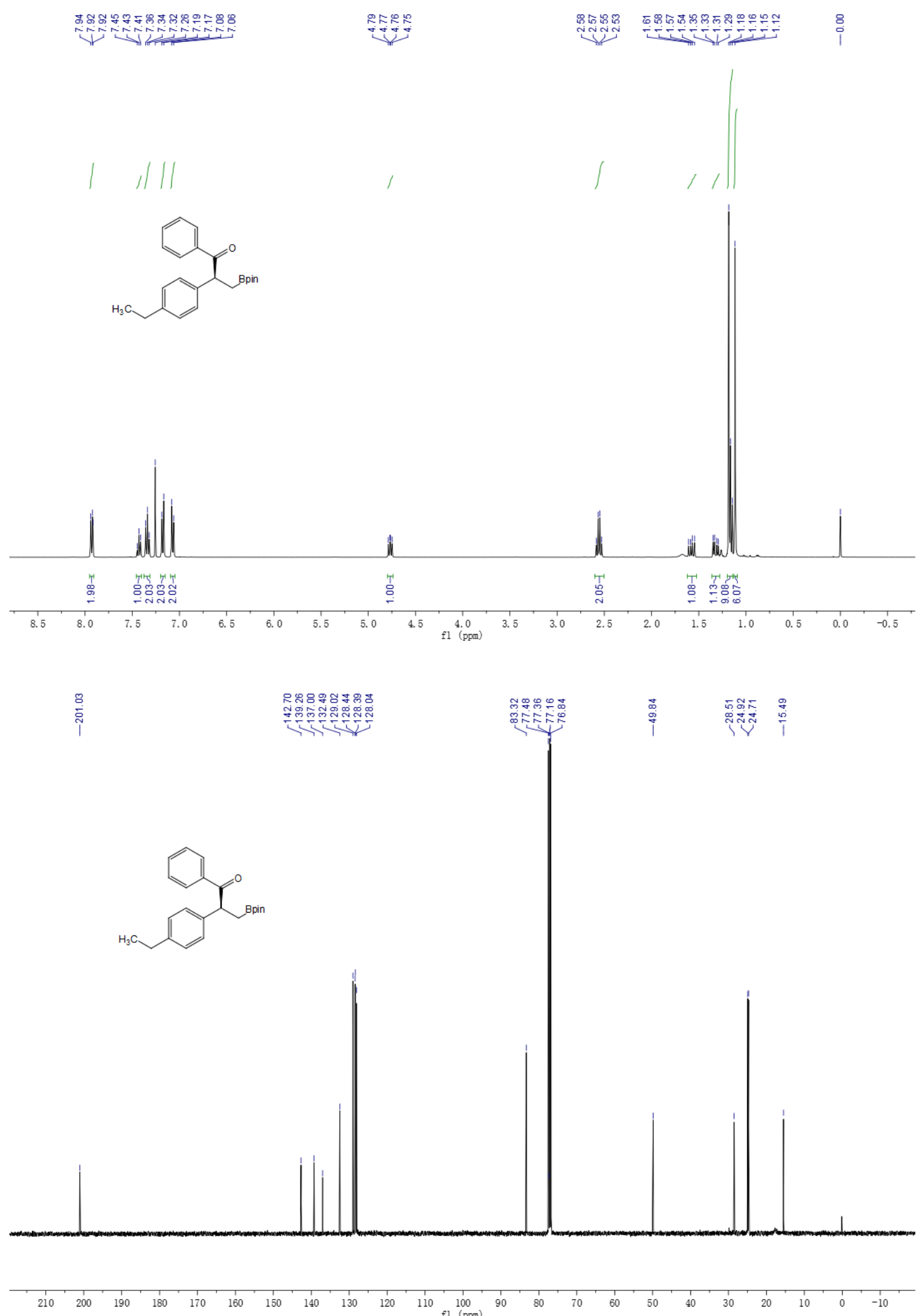
(S)-2-(4-(tert-butyl)phenyl)-1-phenyl-3-(4,4,5,5-tetramethyl-1,3,2-dioxaborolan-2-yl)propan-1-on e (3d): ${ }^{1} \mathrm{H}$ NMR $400 \mathrm{MHz},{ }^{13} \mathrm{C}\{1 \mathrm{H}\}$ NMR $101 \mathrm{MHz}, \mathrm{CDCl}_{3}$

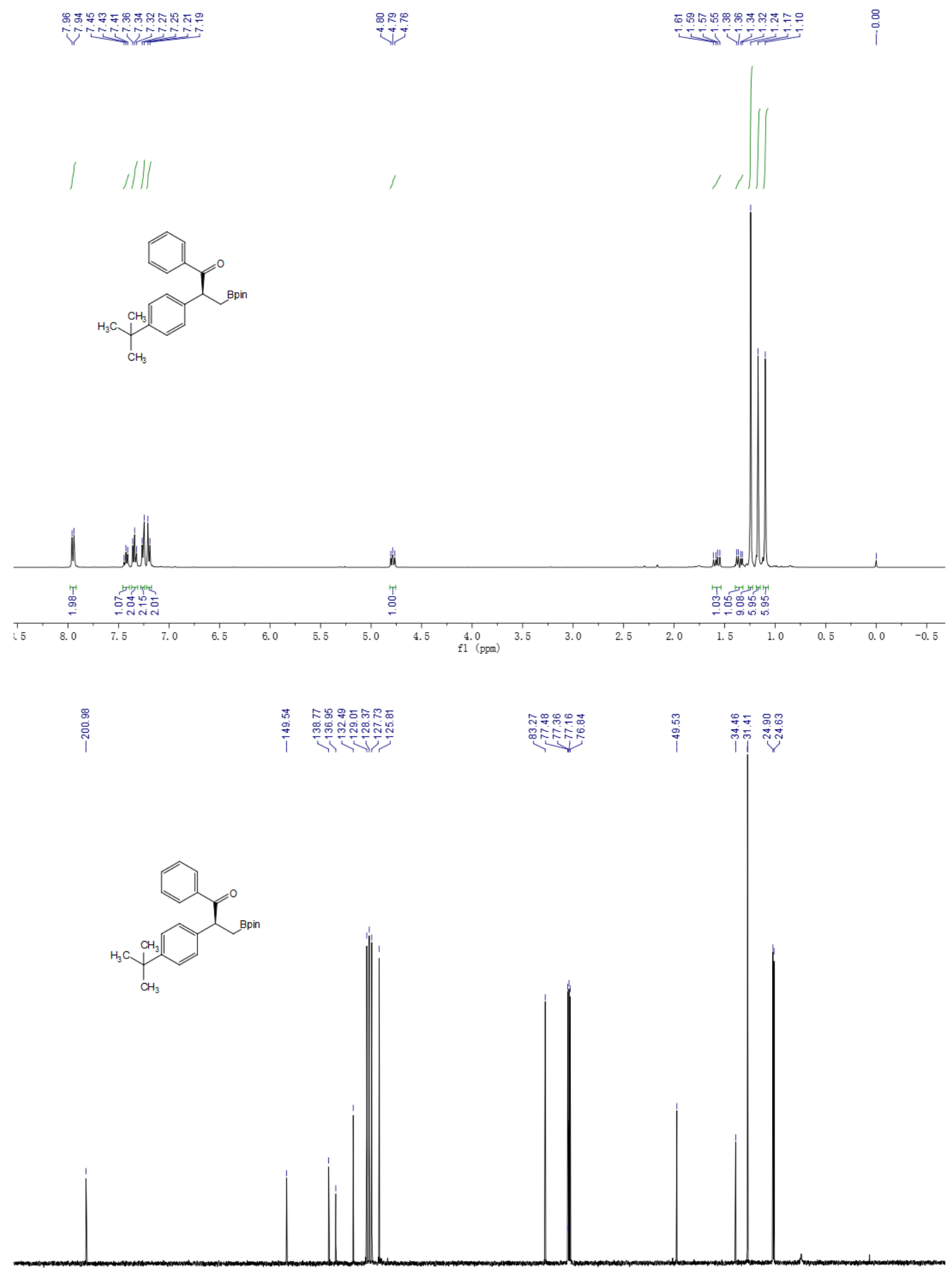

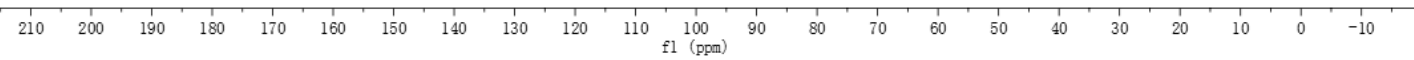


(S)-2-([1,1'-biphenyl]-4-yl)-1-phenyl-3-(4,4,5,5-tetramethyl-1,3,2-dioxaborolan-2-yl)propan-1-one (3e): ${ }^{1} \mathrm{H}$ NMR $400 \mathrm{MHz},{ }^{13} \mathrm{C}\{1 \mathrm{H}\} \mathrm{NMR} 101 \mathrm{MHz}, \mathrm{CDCl}_{3}$

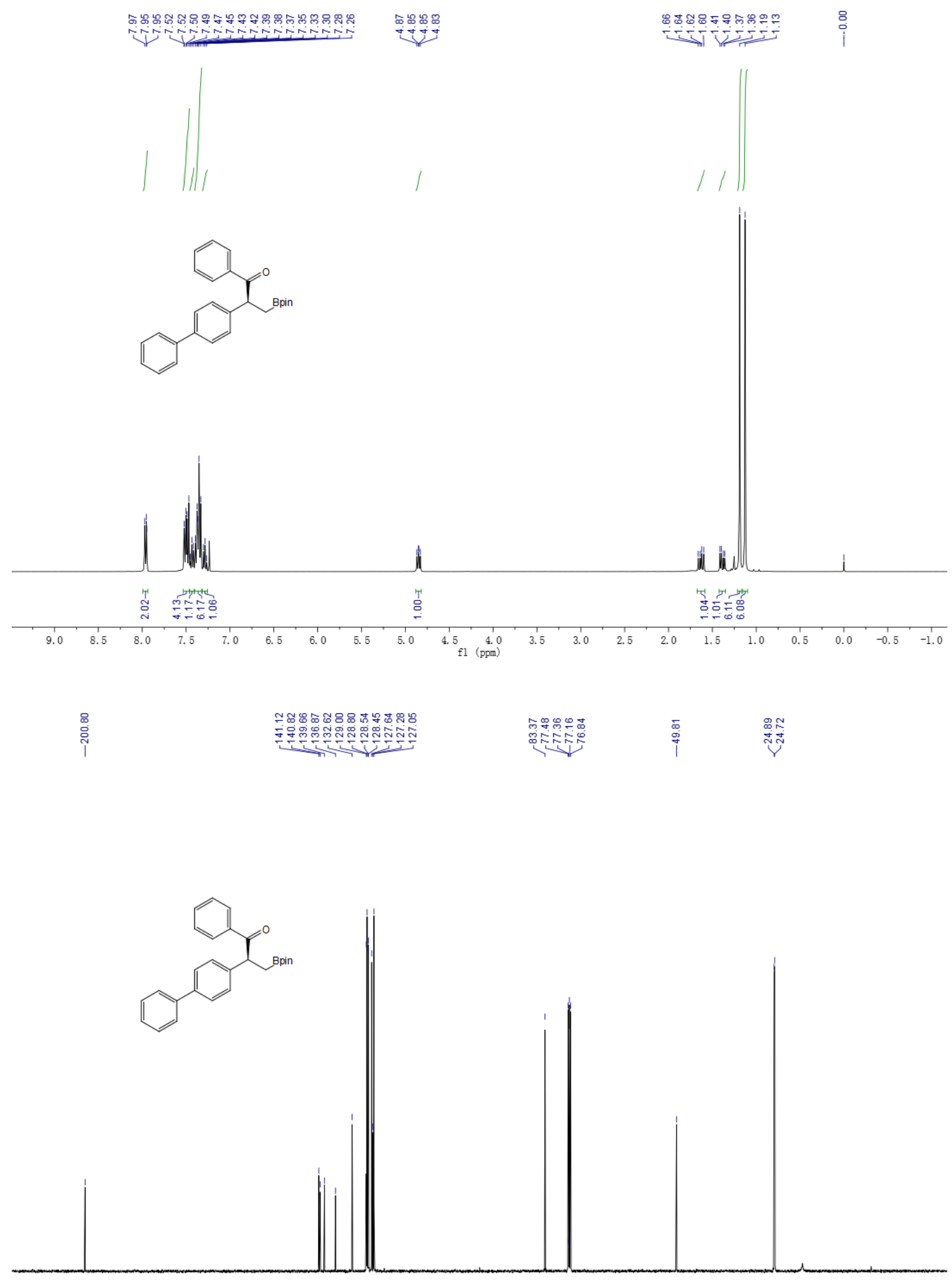

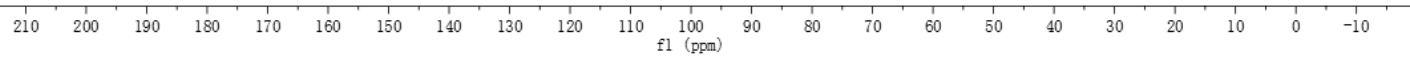


(S)-1-phenyl-2-(4-(phenylethynyl)phenyl)-3-(4,4,5,5-tetramethyl-1,3,2-dioxaborolan-2-yl)propan1-one (3f): ${ }^{1} \mathrm{H}$ NMR $400 \mathrm{MHz},{ }^{13} \mathrm{C}\{1 \mathrm{H}\} \mathrm{NMR} 101 \mathrm{MHz}, \mathrm{CDCl}_{3}$
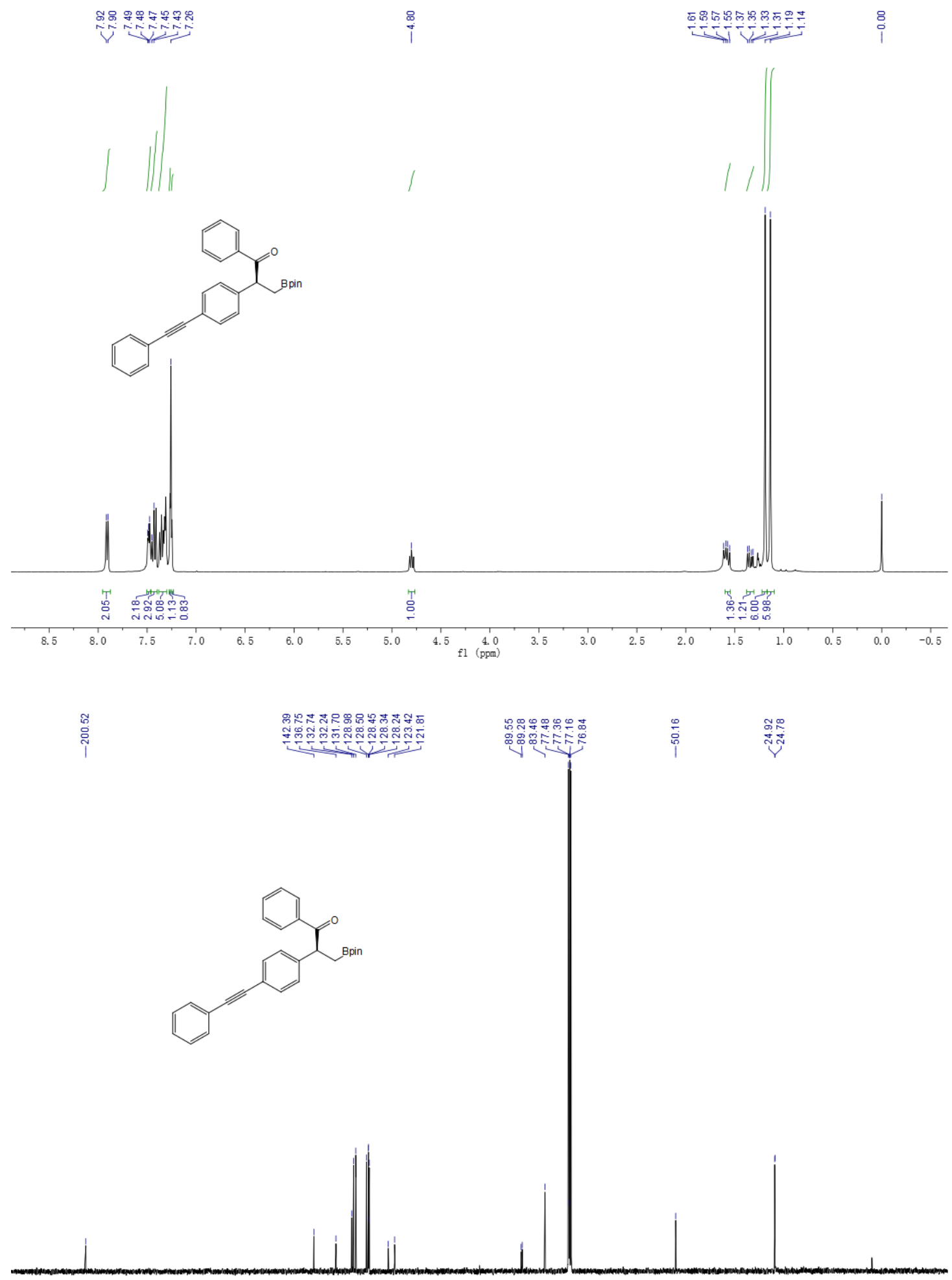

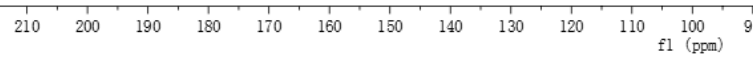


(S)-2-(4-methoxyphenyl)-1-phenyl-3-(4,4,5,5-tetramethyl-1,3,2-dioxaborolan-2-yl)propan-1-one (3g): ${ }^{1} \mathrm{H}$ NMR $400 \mathrm{MHz},{ }^{13} \mathrm{C}\{1 \mathrm{H}\} \mathrm{NMR} 101 \mathrm{MHz}, \mathrm{CDCl}_{3}$

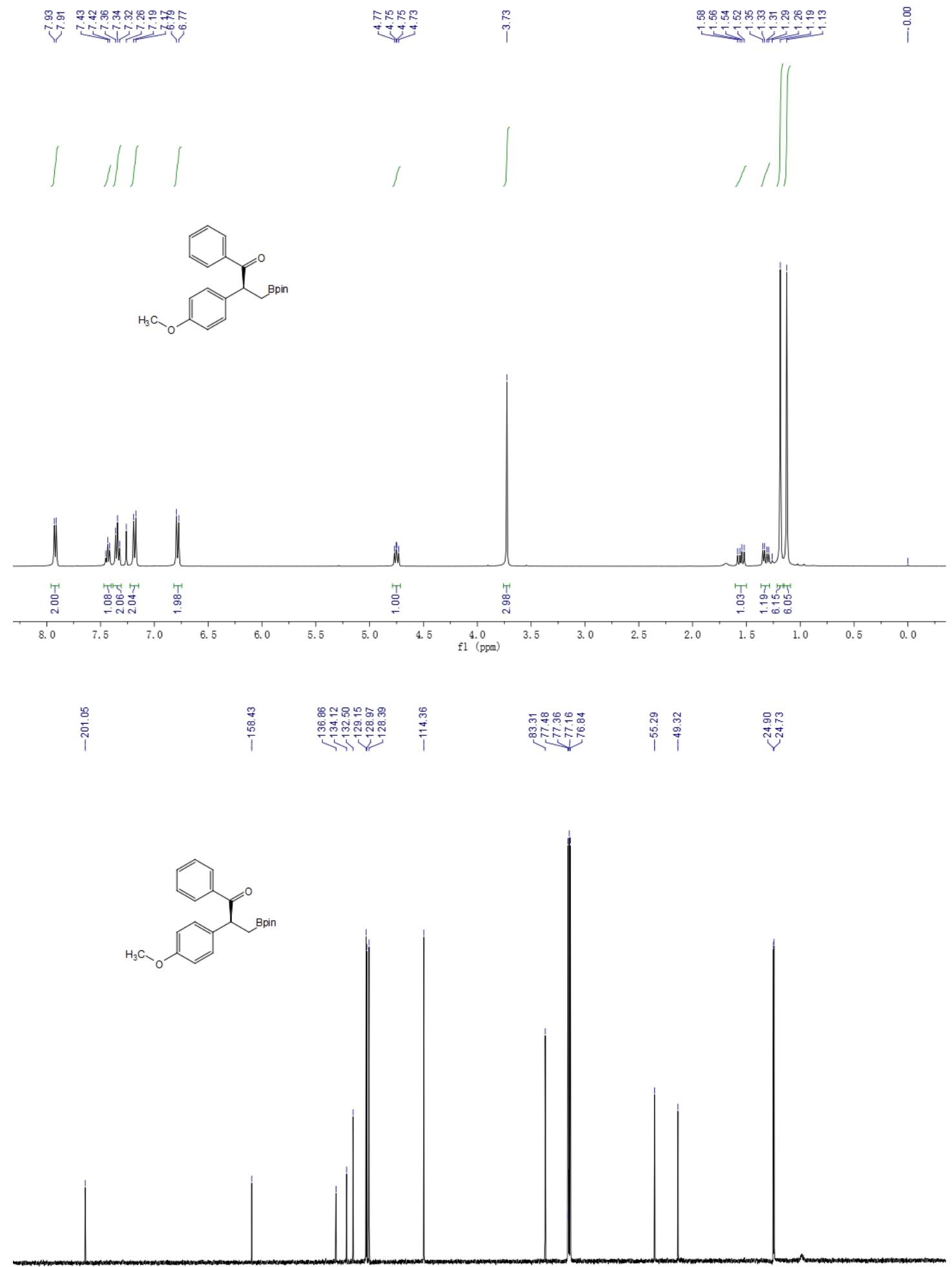

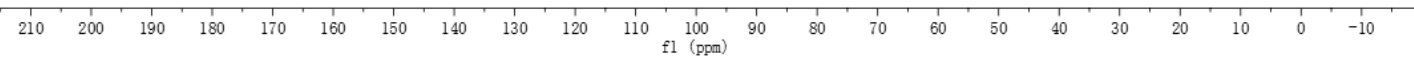


(S)-2-(4-fluorophenyl)-1-phenyl-3-(4,4,5,5-tetramethyl-1,3,2-dioxaborolan-2-yl)propan-1-one (3h): ${ }^{1} \mathrm{H}$ NMR $400 \mathrm{MHz},{ }^{13} \mathrm{C}\{1 \mathrm{H}\} \mathrm{NMR} 101 \mathrm{MHz},{ }^{19} \mathrm{~F} \mathrm{NMR} 376 \mathrm{MHz}, \mathrm{CDCl}_{3}$
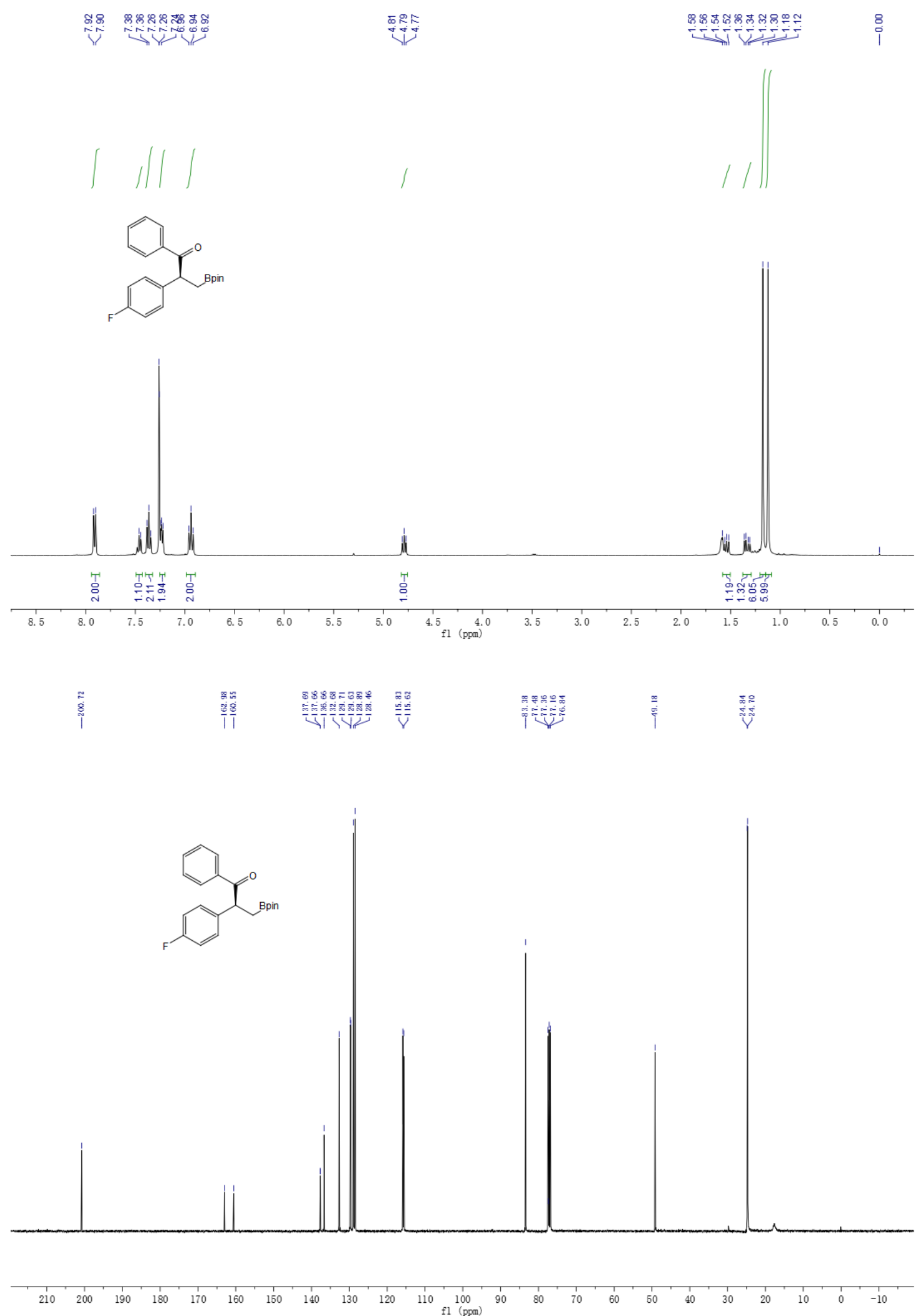
$\stackrel{\frac{1}{6}}{\frac{N}{j}}$
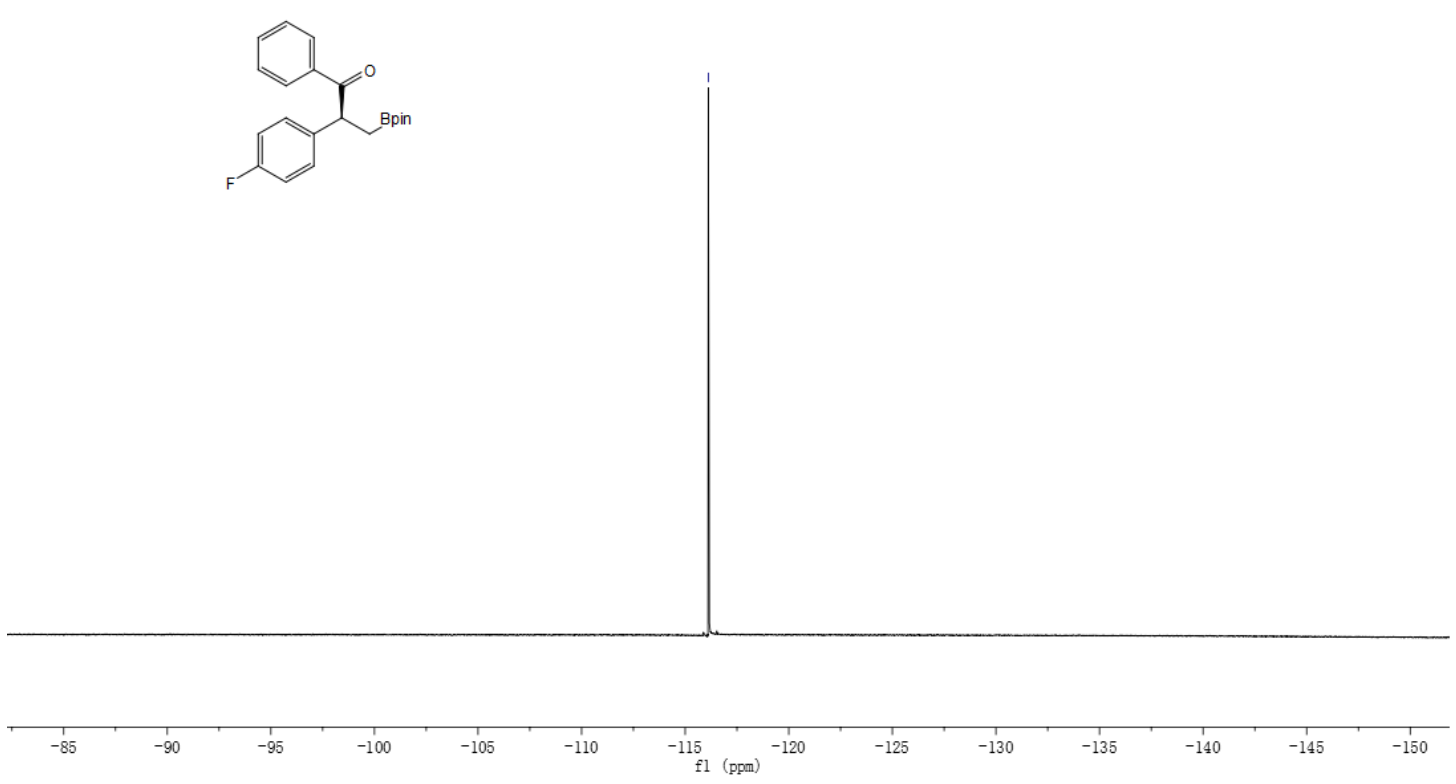

S48 
(S)-2-(4-chlorophenyl)-1-phenyl-3-(4,4,5,5-tetramethyl-1,3,2-dioxaborolan-2-yl)propan-1-one (3i): ${ }^{1} \mathrm{H}$ NMR $400 \mathrm{MHz},{ }^{13} \mathrm{C}\{1 \mathrm{H}\}$ NMR $101 \mathrm{MHz}, \mathrm{CDCl}_{3}$
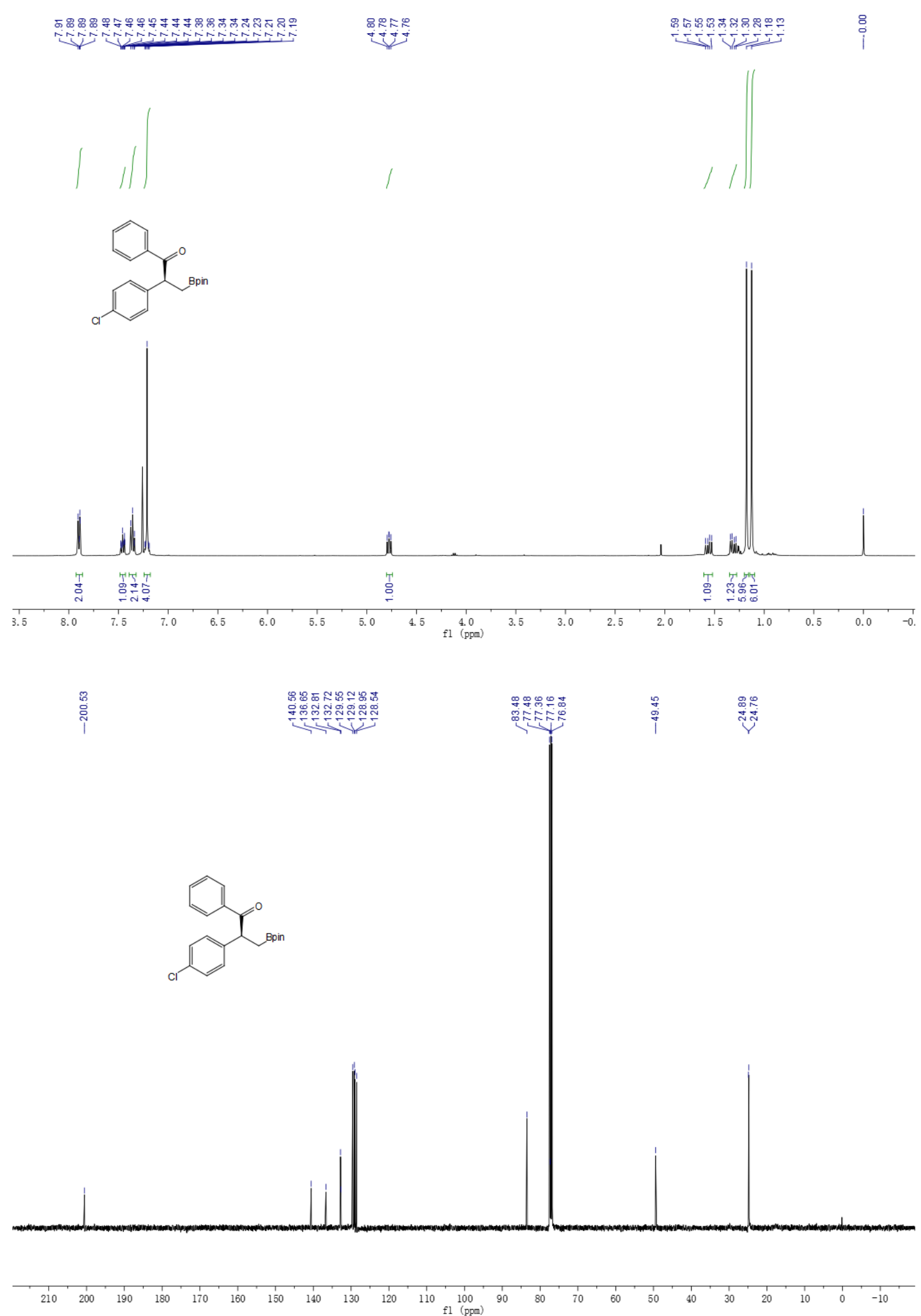
(S)-2-(4-bromophenyl)-1-phenyl-3-(4,4,5,5-tetramethyl-1,3,2-dioxaborolan-2-yl)propan-1-one (3j): ${ }^{1} \mathrm{H}$ NMR $400 \mathrm{MHz},{ }^{13} \mathrm{C}\{1 \mathrm{H}\}$ NMR $101 \mathrm{MHz}, \mathrm{CDCl}_{3}$
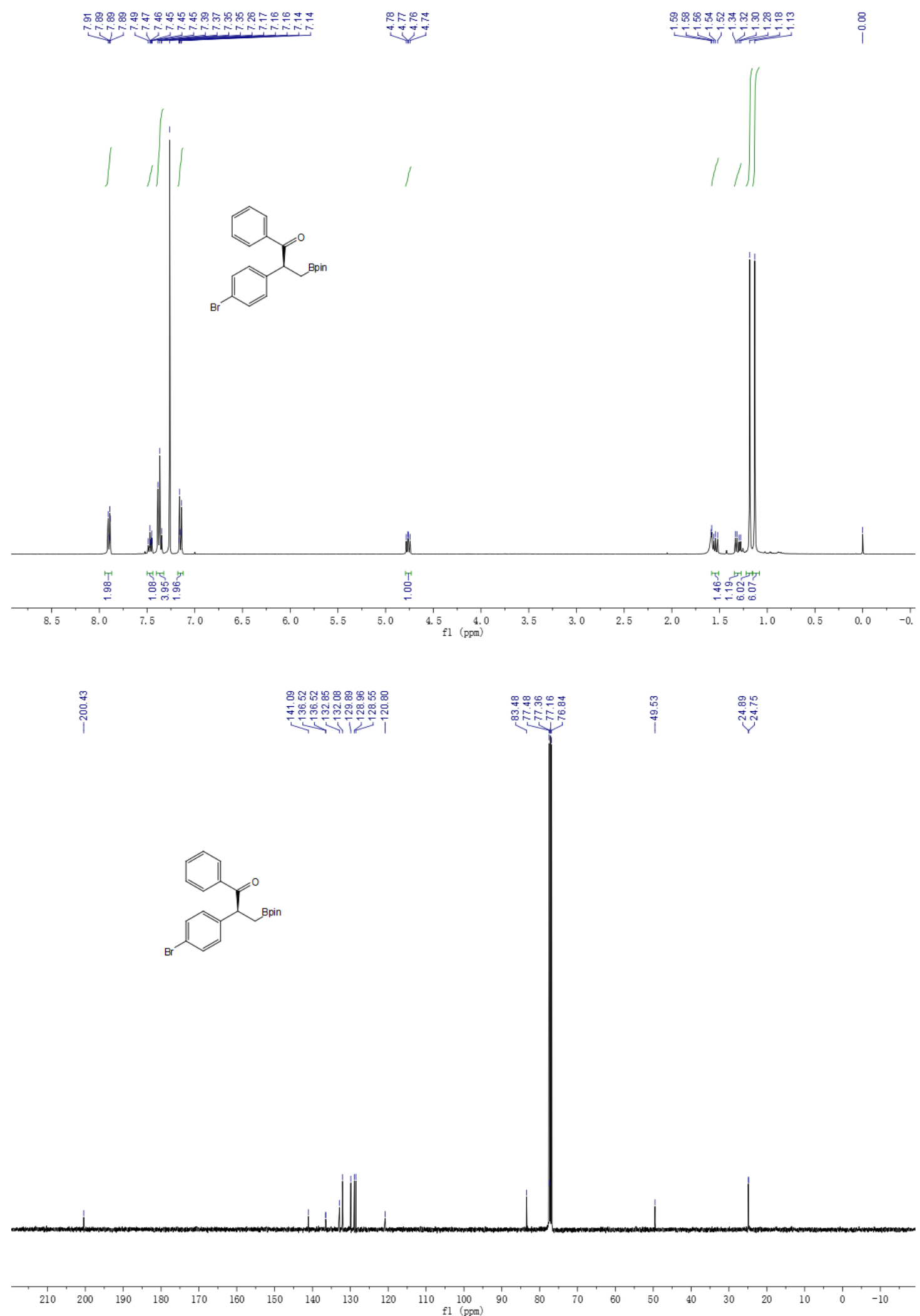
(S)-1-phenyl-3-(4,4,5,5-tetramethyl-1,3,2-dioxaborolan-2-yl)-2-(m-tolyl)propan-1-one $\quad$ (3k): $\quad{ }^{1} \mathrm{H}$ NMR $400 \mathrm{MHz},{ }^{13} \mathrm{C}\{1 \mathrm{H}\}$ NMR $101 \mathrm{MHz}, \mathrm{CDCl}_{3}$
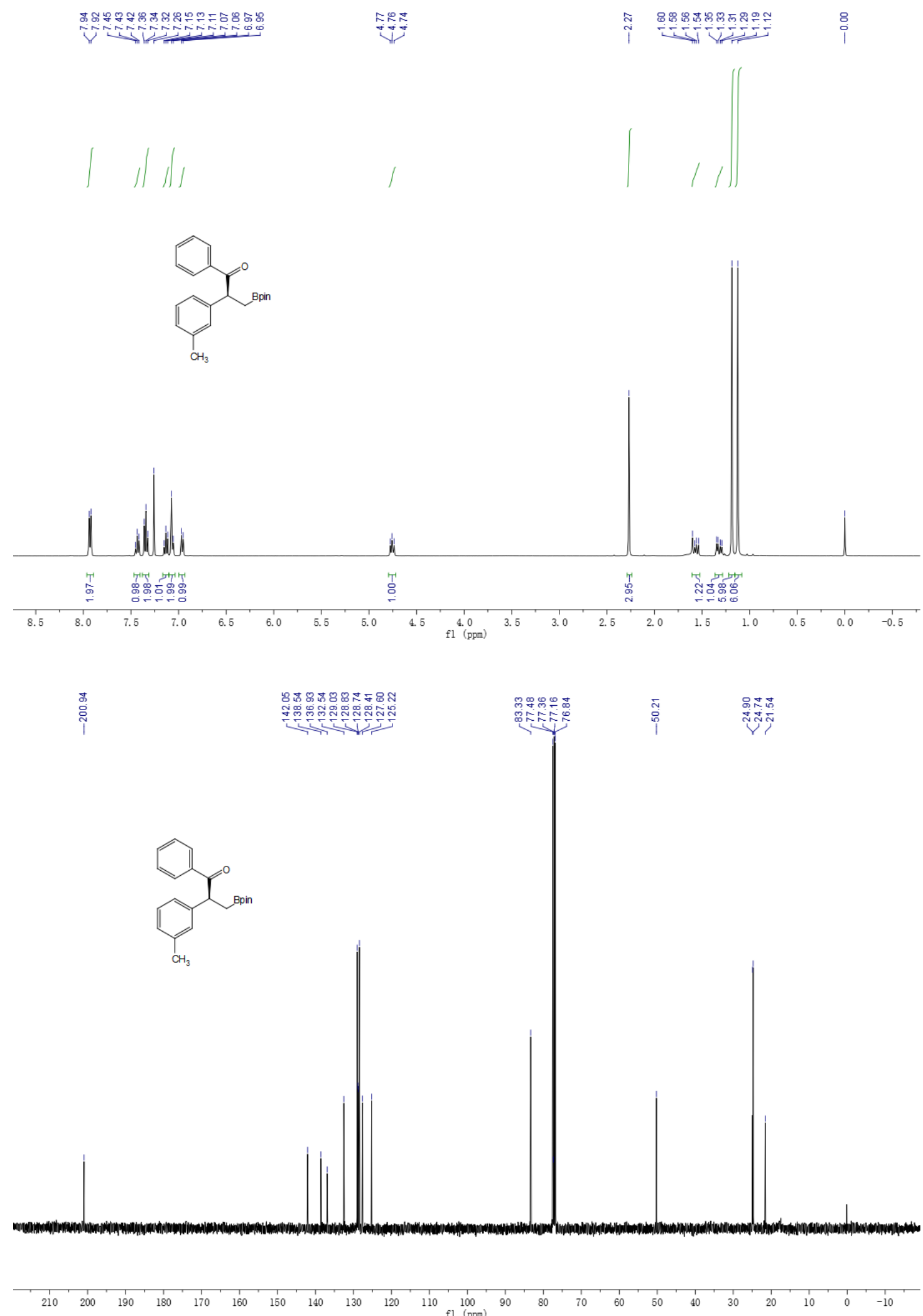
(S)-2-(3-fluorophenyl)-1-phenyl-3-(4,4,5,5-tetramethyl-1,3,2-dioxaborolan-2-yl)propan-1-one (31): ${ }^{1} \mathrm{H}$ NMR $400 \mathrm{MHz},{ }^{13} \mathrm{C}\{1 \mathrm{H}\}$ NMR $101 \mathrm{MHz},{ }^{19} \mathrm{~F}$ NMR $376 \mathrm{MHz}, \mathrm{CDCl}_{3}$
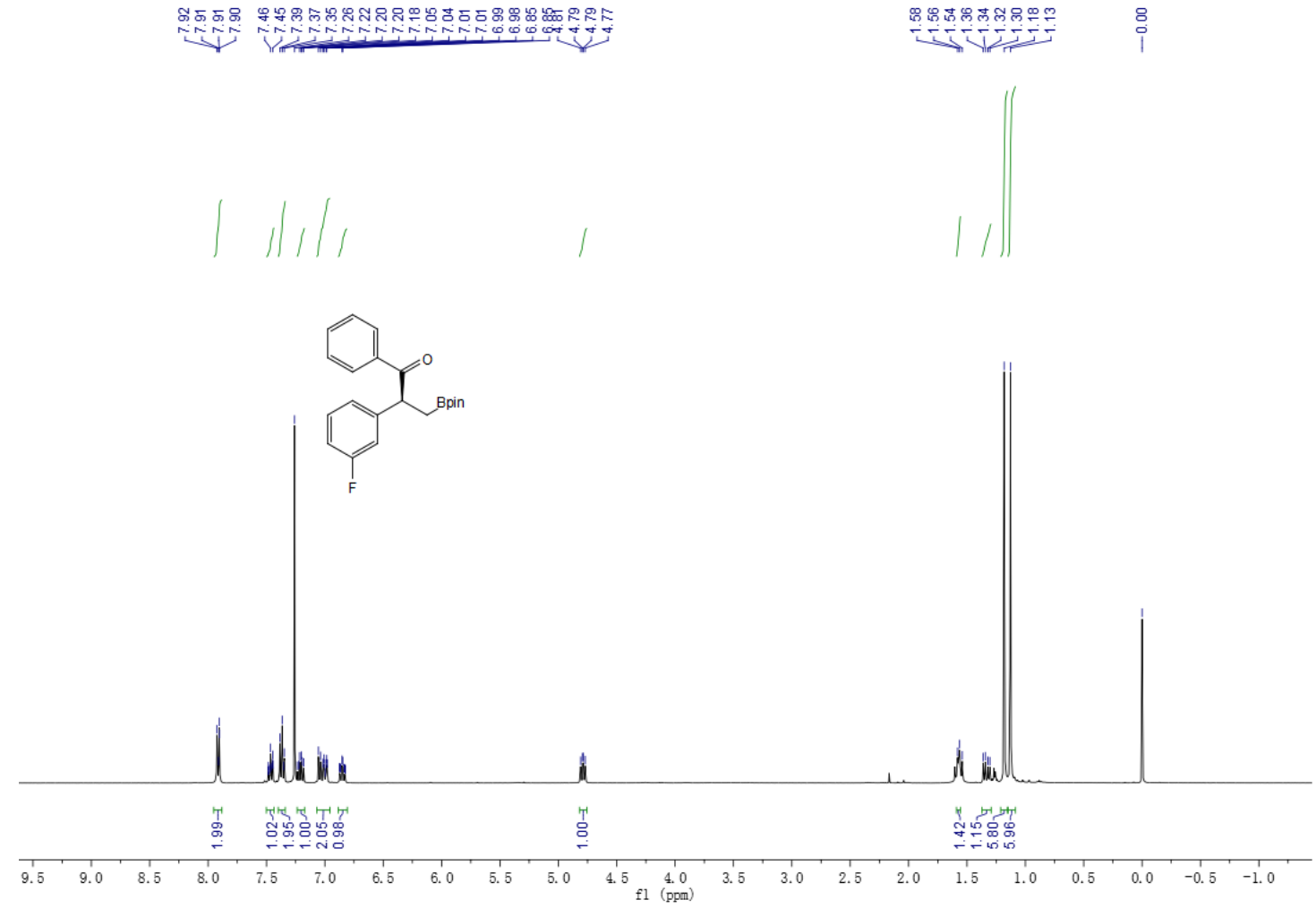

ग्ल

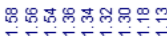

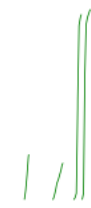
i

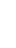

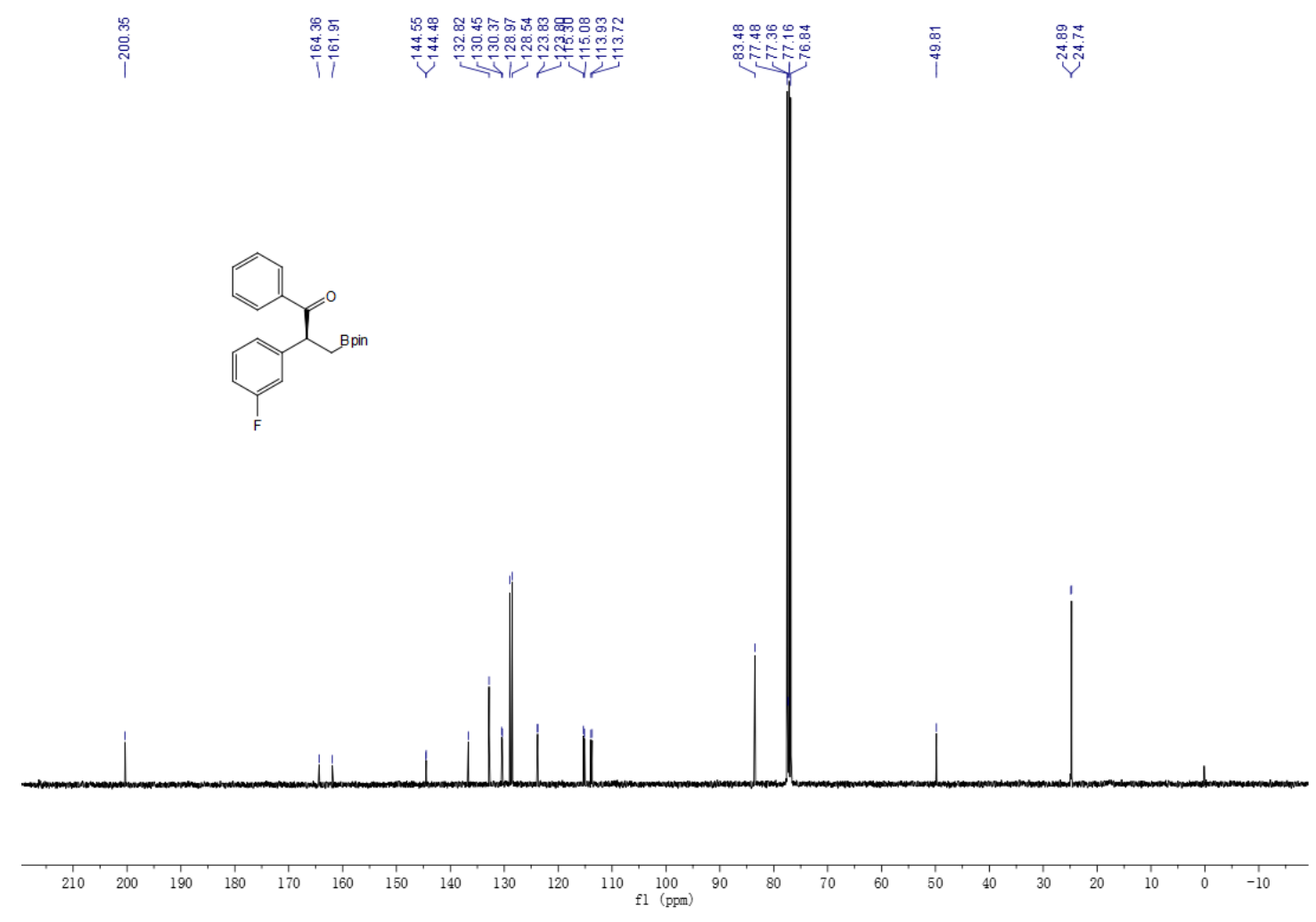

S52 


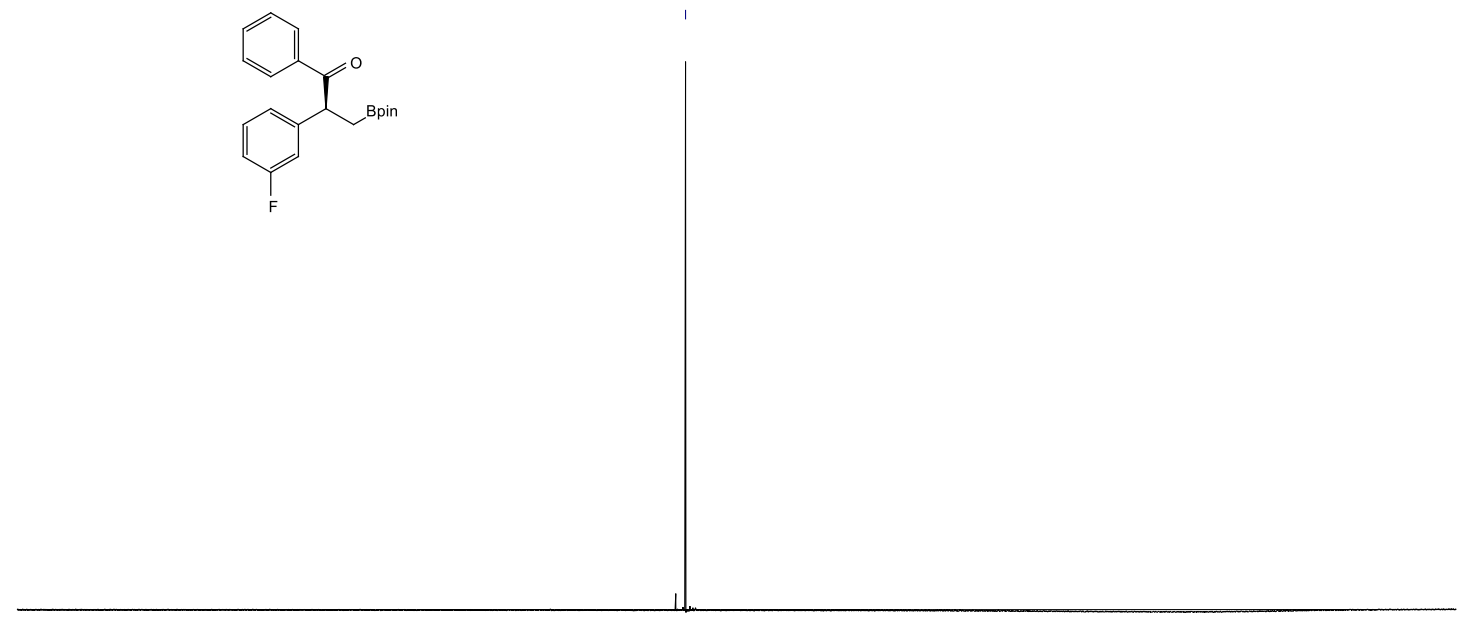

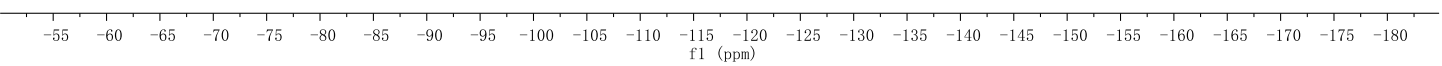


(S)-2-(3-chlorophenyl)-1-phenyl-3-(4,4,5,5-tetramethyl-1,3,2-dioxaborolan-2-yl)propan-1-one (3m): ${ }^{1} \mathrm{H}$ NMR $400 \mathrm{MHz},{ }^{13} \mathrm{C}\{1 \mathrm{H}\} \mathrm{NMR} 101 \mathrm{MHz}, \mathrm{CDCl}_{3}$

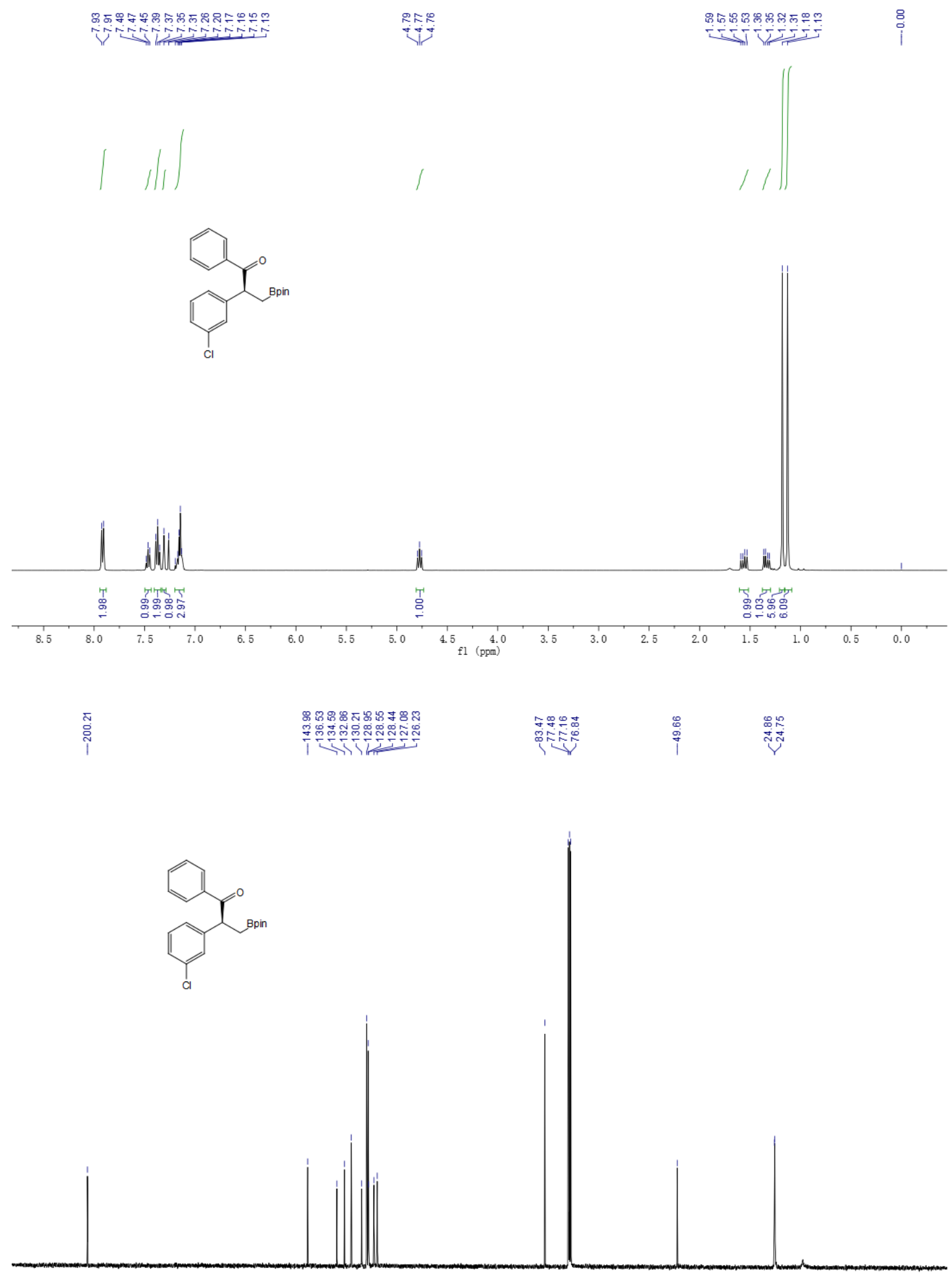

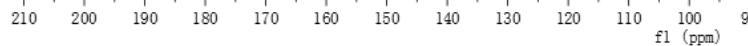


(S)-2-(3-bromophenyl)-1-phenyl-3-(4,4,5,5-tetramethyl-1,3,2-dioxaborolan-2-yl)propan-1-one (3n): ${ }^{1} \mathrm{H}$ NMR $400 \mathrm{MHz},{ }^{13} \mathrm{C}\{1 \mathrm{H}\} \mathrm{NMR} 101 \mathrm{MHz}, \mathrm{CDCl}_{3}$

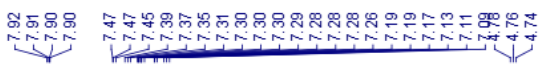

年

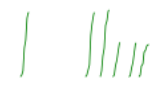

Brin

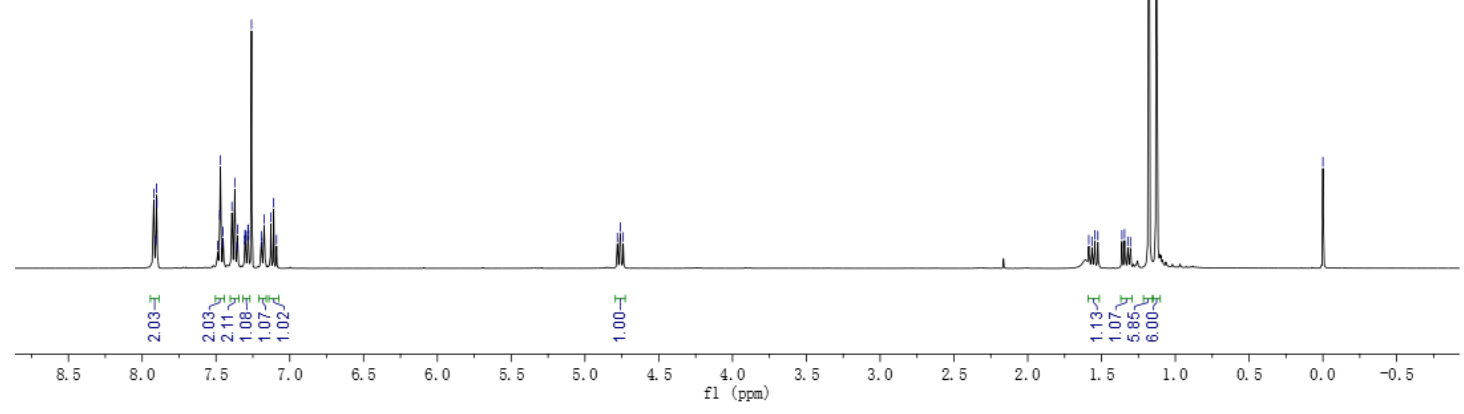

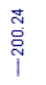

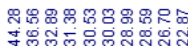

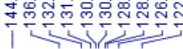

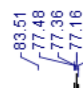

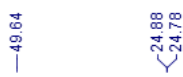
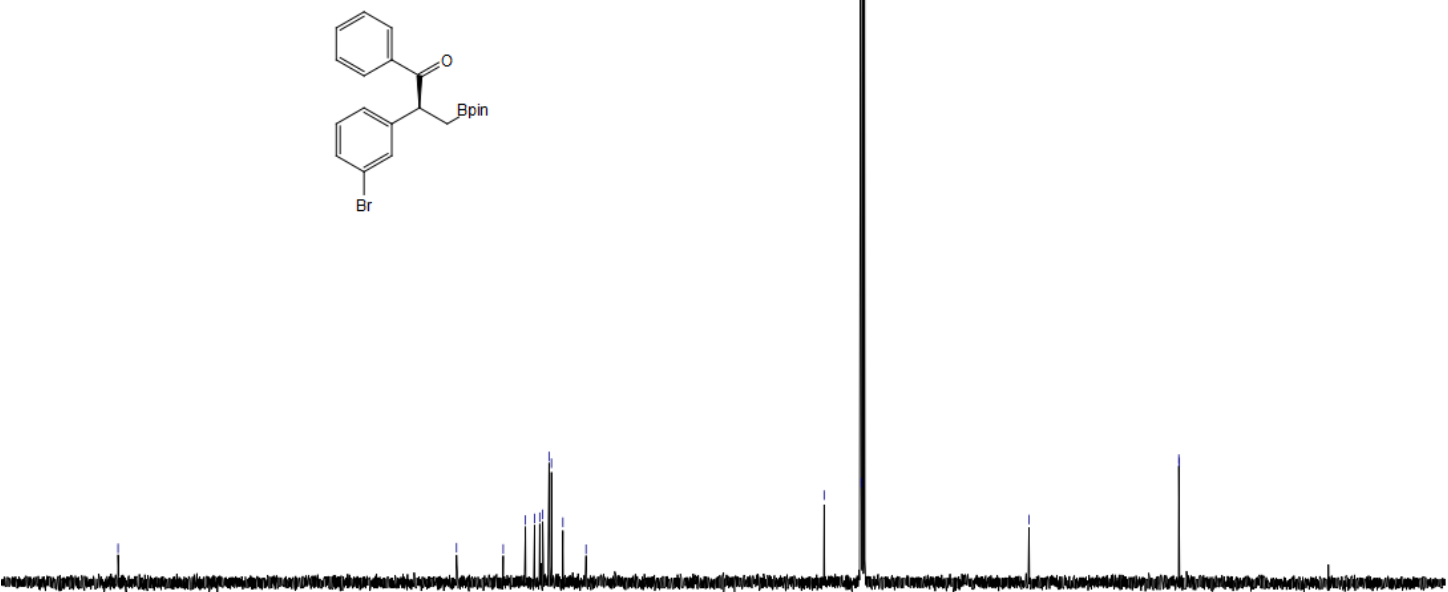

$\begin{array}{llllllllllll}210 & 200 & 190 & 180 & 170 & 160 & 150 & 140 & 130 & 120 & 110 & 100\end{array}$ 
(S)-1-phenyl-3-(4,4,5,5-tetramethyl-1,3,2-dioxaborolan-2-yl)-2-(o-tolyl)propan-1-one

(3o): ${ }^{1} \mathrm{H}$ NMR $400 \mathrm{MHz},{ }^{13} \mathrm{C}\{1 \mathrm{H}\} \mathrm{NMR} 101 \mathrm{MHz}, \mathrm{CDCl}_{3}$
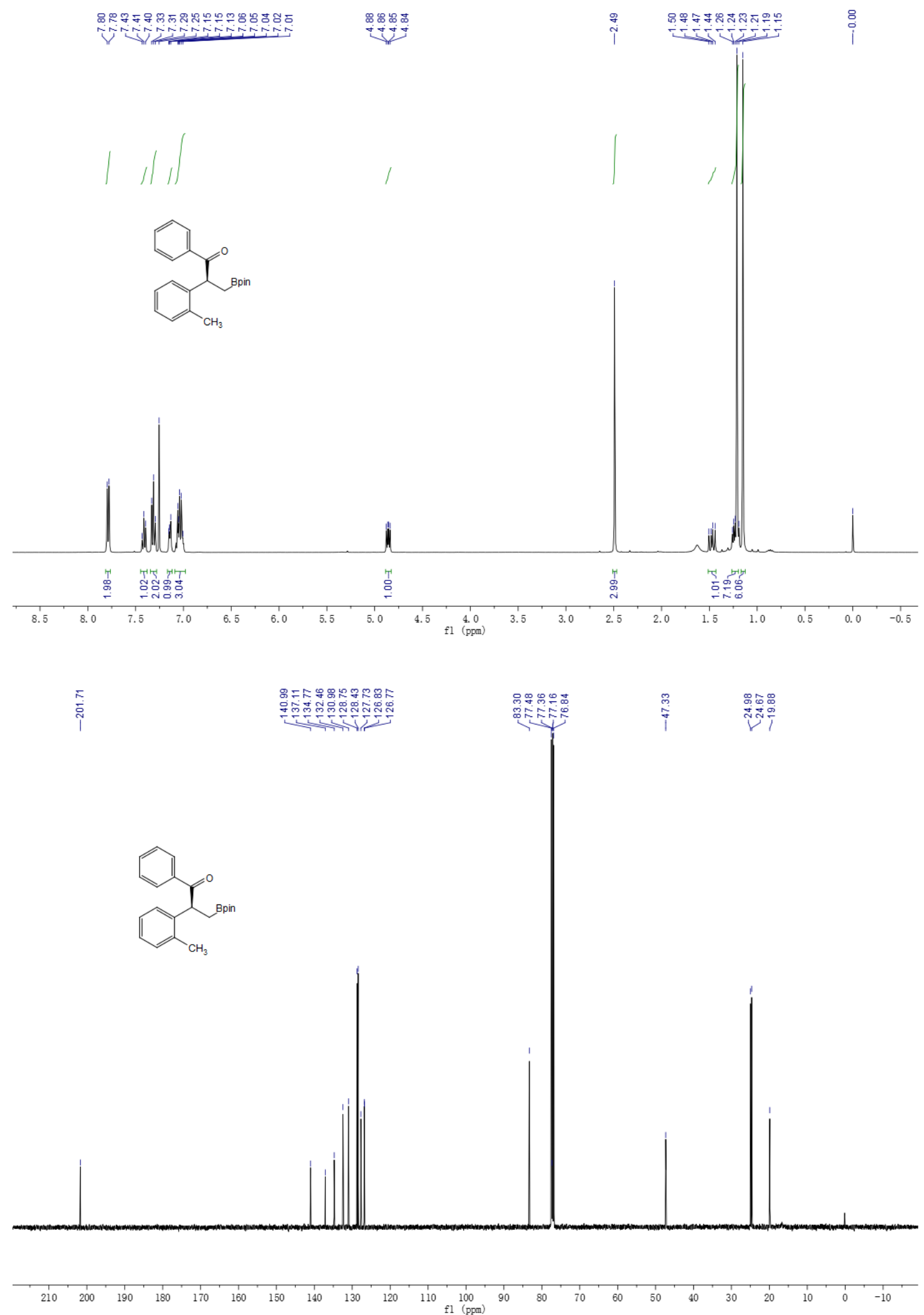
(S)-2-(2-fluorophenyl)-1-phenyl-3-(4,4,5,5-tetramethyl-1,3,2-dioxaborolan-2-yl)propan-1-one (3p): ${ }^{1} \mathrm{H}$ NMR $400 \mathrm{MHz},{ }^{13} \mathrm{C}\{1 \mathrm{H}\}$ NMR $101 \mathrm{MHz},{ }^{19} \mathrm{~F}$ NMR $376 \mathrm{MHz}, \mathrm{CDCl}_{3}$

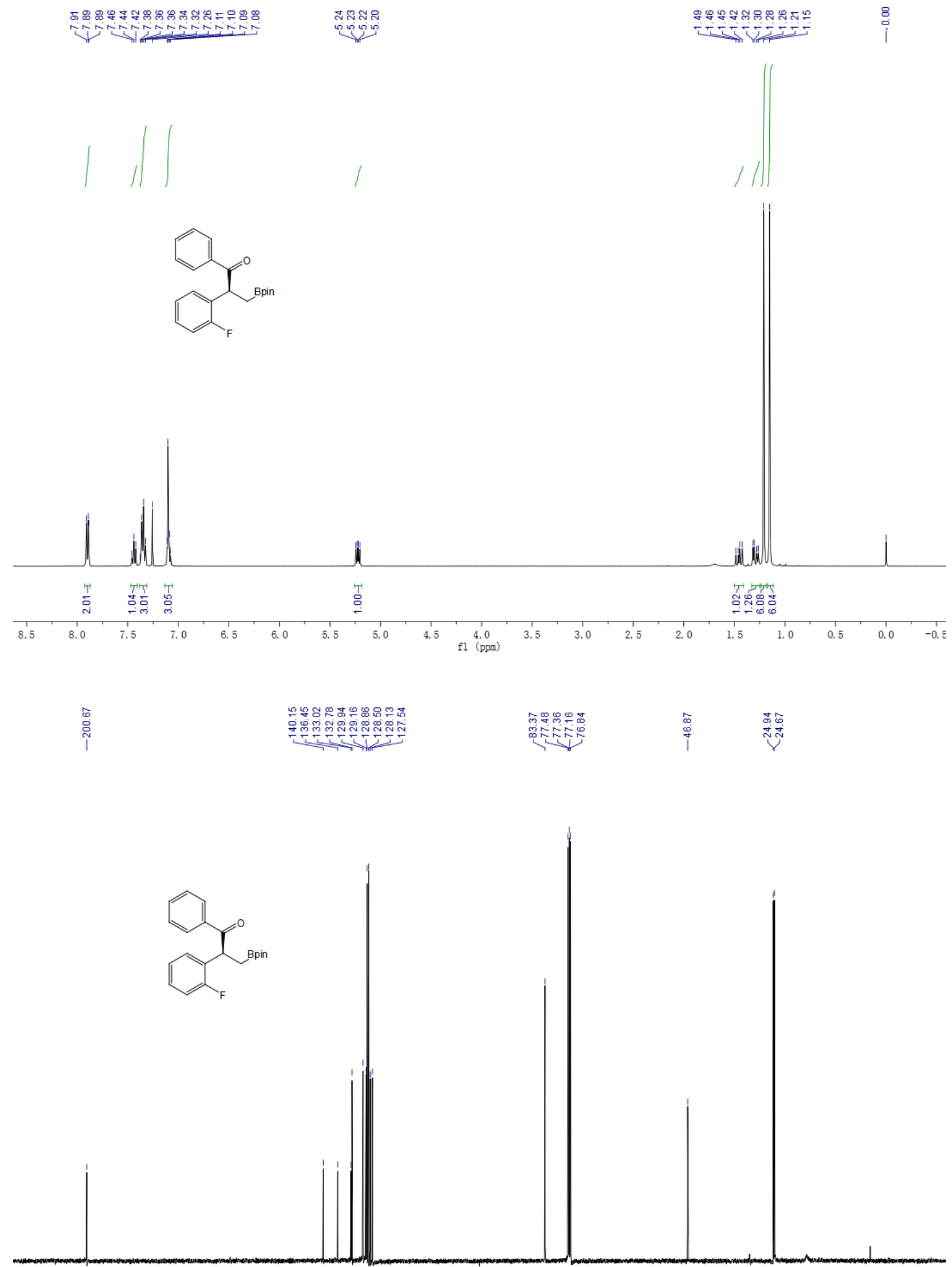

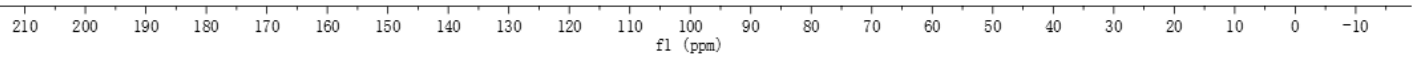



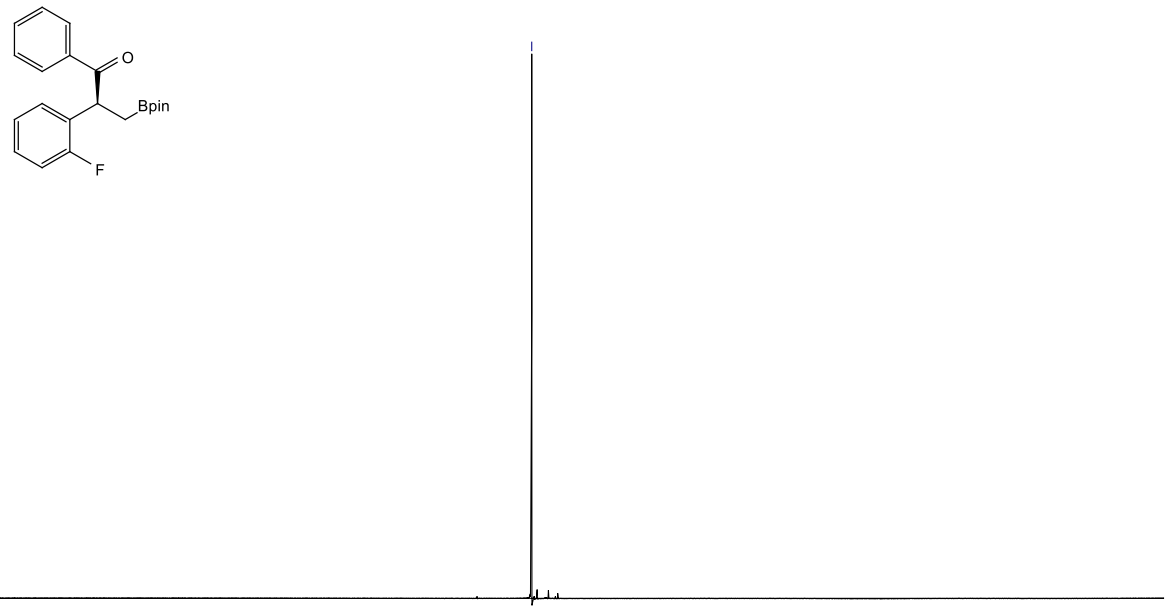

$\begin{array}{r}10 \\ \hline 1\end{array}$ 
(S)-2-(2-chlorophenyl)-1-phenyl-3-(4,4,5,5-tetramethyl-1,3,2-dioxaborolan-2-yl)propan-1-one (3q): ${ }^{1} \mathrm{H}$ NMR $400 \mathrm{MHz},{ }^{13} \mathrm{C}\{1 \mathrm{H}\}$ NMR $101 \mathrm{MHz}, \mathrm{CDCl}_{3}$

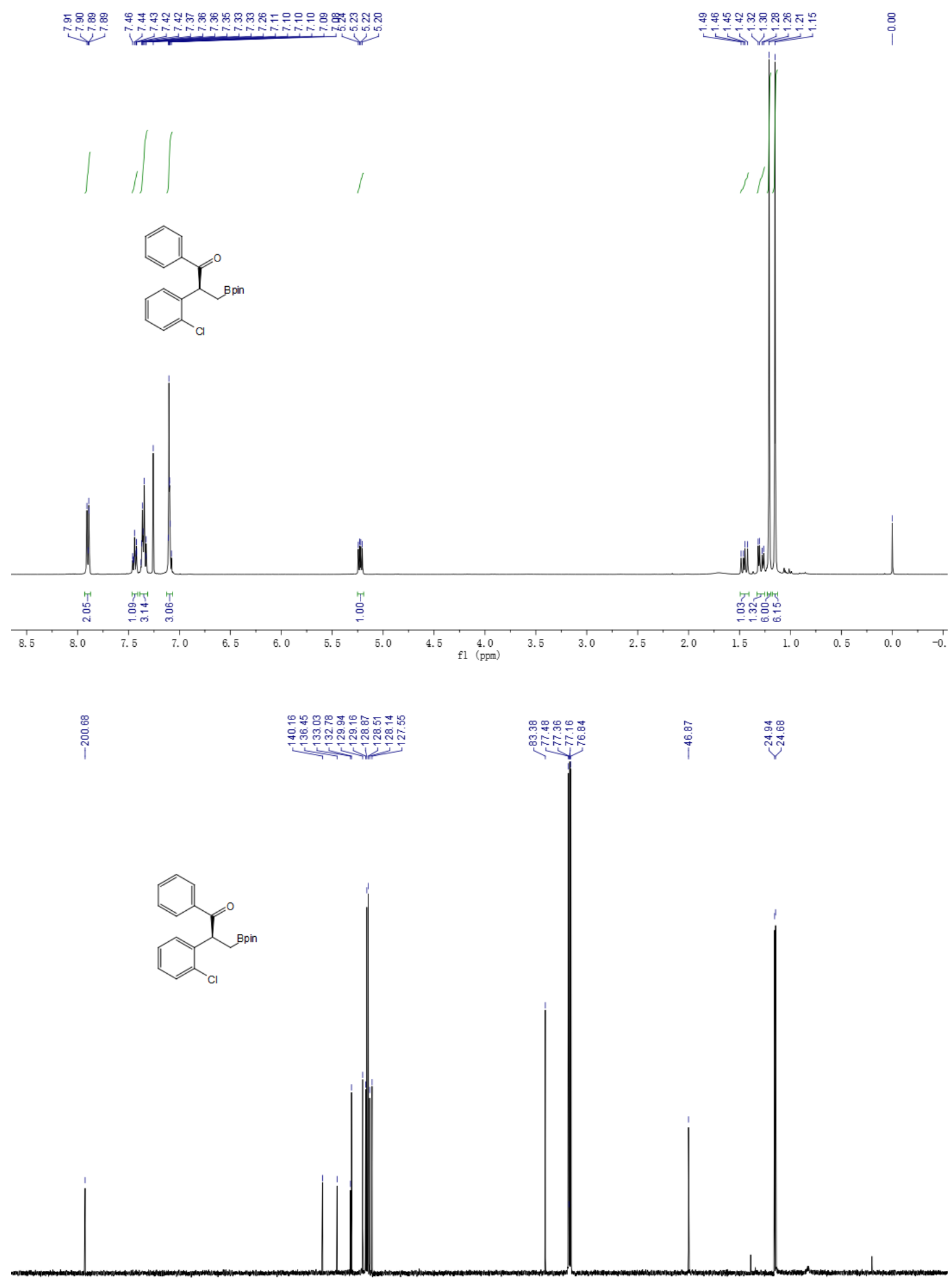

$\begin{array}{llllllllllll}210 & 200 & 190 & 180 & 170 & 160 & 150 & 140 & 130 & 120 & 110 & 100 \\ \mathrm{fl}(\mathrm{ppm})\end{array}$ 
(S)-2-(2-(methoxymethoxy)phenyl)-1-phenyl-3-(4,4,5,5-tetramethyl-1,3,2-dioxaborolan-2-yl)prop an-1-one (3r): ${ }^{1} \mathrm{H}$ NMR $400 \mathrm{MHz},{ }^{13} \mathrm{C}\{1 \mathrm{H}\}$ NMR $101 \mathrm{MHz}, \mathrm{CDCl}_{3}$

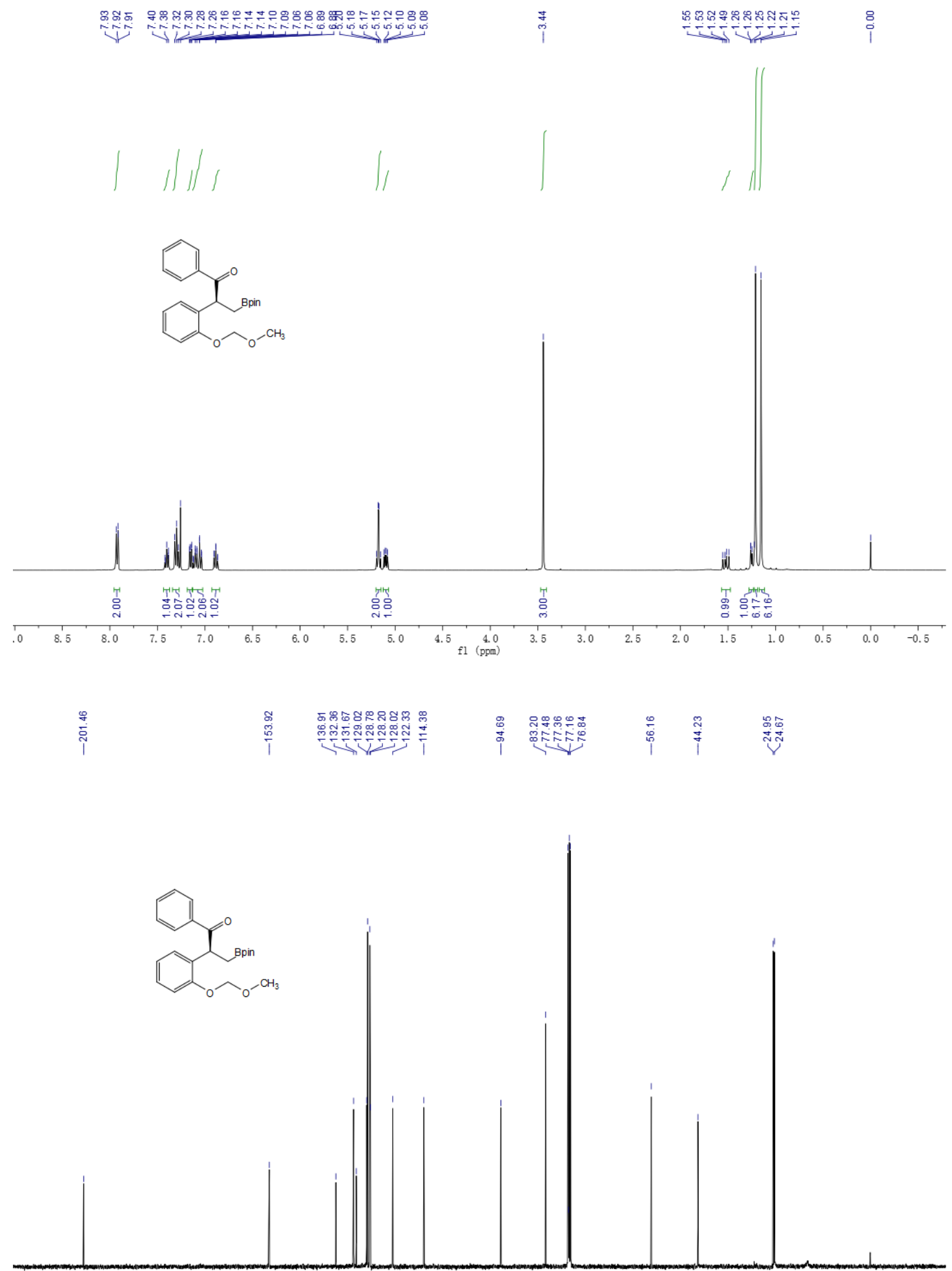

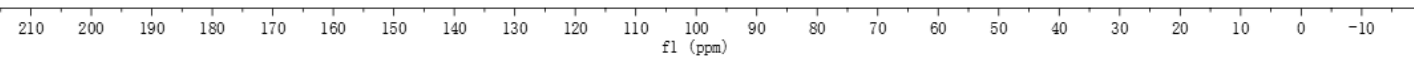


phenyl((1S,2S)-2-(4,4,5,5-tetramethyl-1,3,2-dioxaborolan-2-yl)-2,3-dihydro-1H-inden-1-yl)metha none (3s): ${ }^{1} \mathrm{H}$ NMR $400 \mathrm{MHz},{ }^{13} \mathrm{C}\{1 \mathrm{H}\}$ NMR $101 \mathrm{MHz}, \mathrm{CDCl}_{3}$
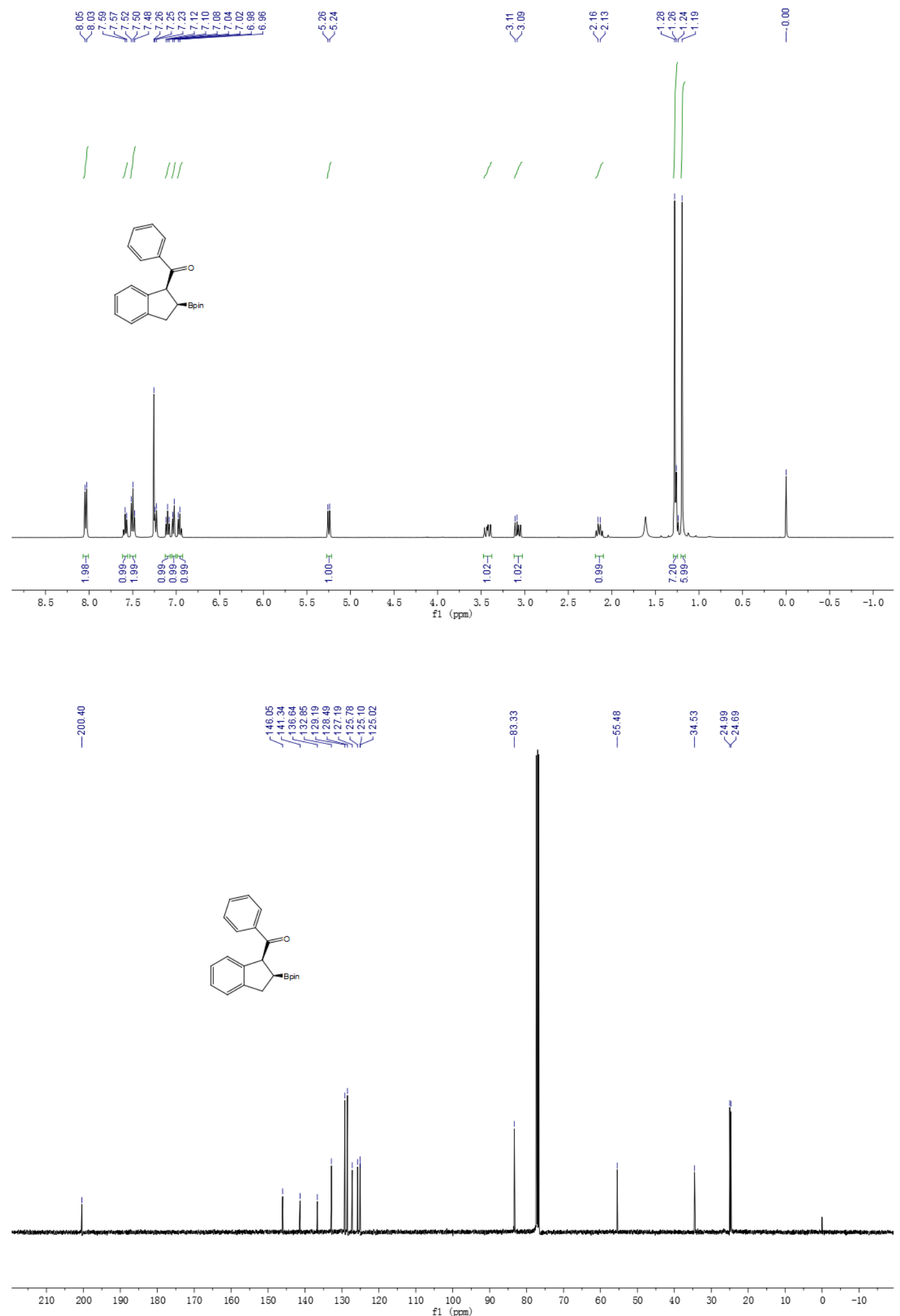
((1S,2S)-4-bromo-2-(4,4,5,5-tetramethyl-1,3,2-dioxaborolan-2-yl)-2,3-dihydro-1H-inden-1-yl)(phe nyl)methanone (3t): ${ }^{1} \mathrm{H}$ NMR $400 \mathrm{MHz},{ }^{13} \mathrm{C}\{1 \mathrm{H}\} \mathrm{NMR} 101 \mathrm{MHz}, \mathrm{CDCl}_{3}$
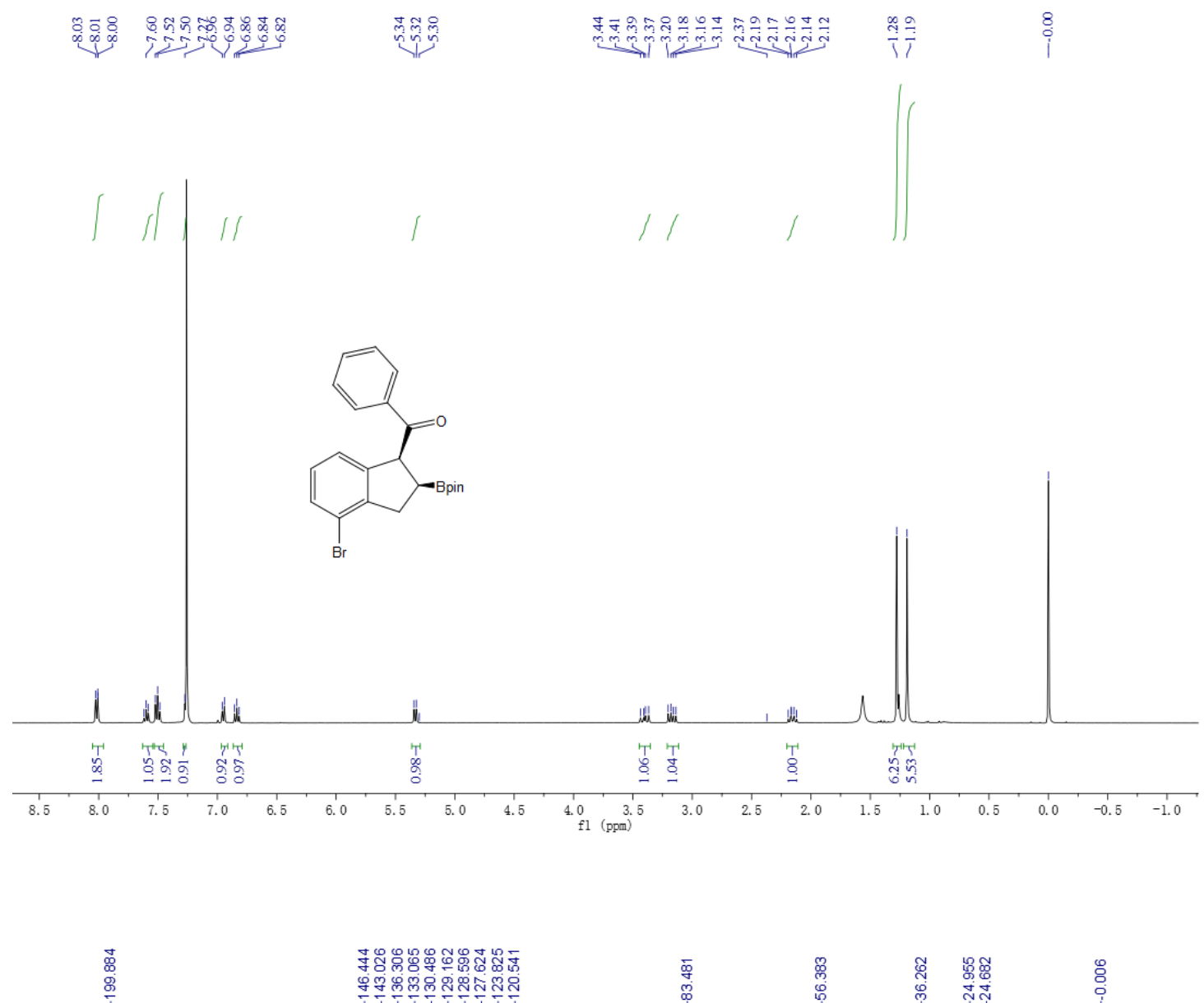

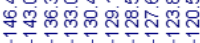

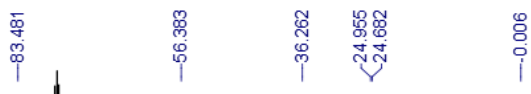

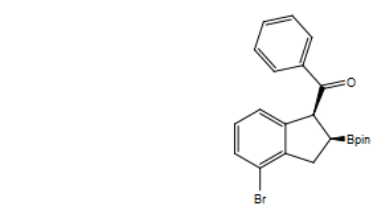

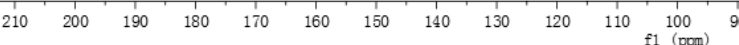


((1S,2S)-6-chloro-2-(4,4,5,5-tetramethyl-1,3,2-dioxaborolan-2-yl)-2,3-dihydro-1H-inden-1-yl)(phe nyl)methanone (3u): ${ }^{1} \mathrm{H}$ NMR $400 \mathrm{MHz},{ }^{13} \mathrm{C}\{1 \mathrm{H}\}$ NMR $101 \mathrm{MHz}, \mathrm{CDCl}_{3}$
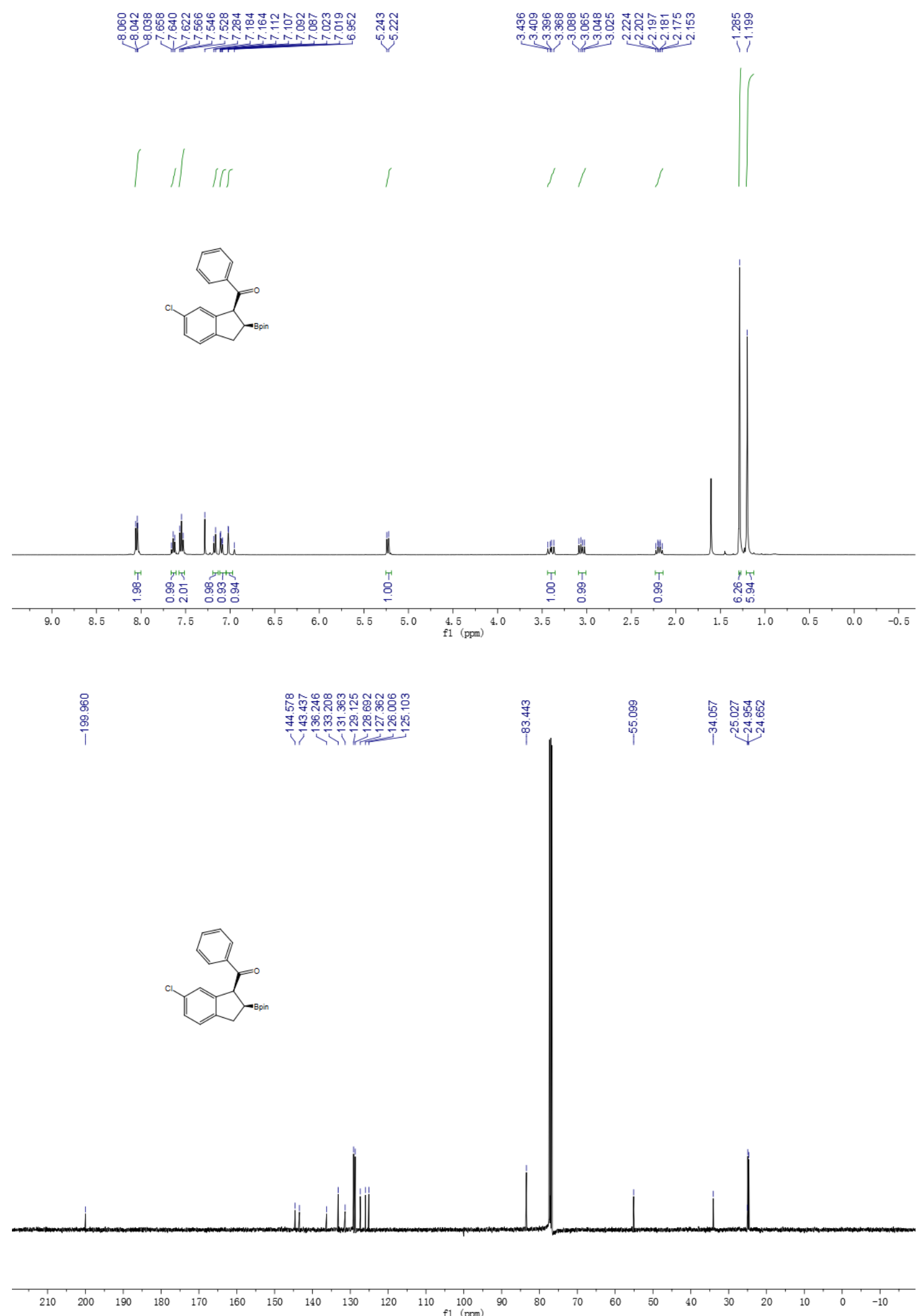
((1S,2S)-4,7-dimethyl-2-(4,4,5,5-tetramethyl-1,3,2-dioxaborolan-2-yl)-2,3-dihydro-1H-inden-1-yl)( phenyl)methanone (3v) ${ }^{1} \mathrm{H}$ NMR $400 \mathrm{MHz},{ }^{13} \mathrm{C}\{1 \mathrm{H}\}$ NMR $101 \mathrm{MHz}, \mathrm{CDCl}_{3}$
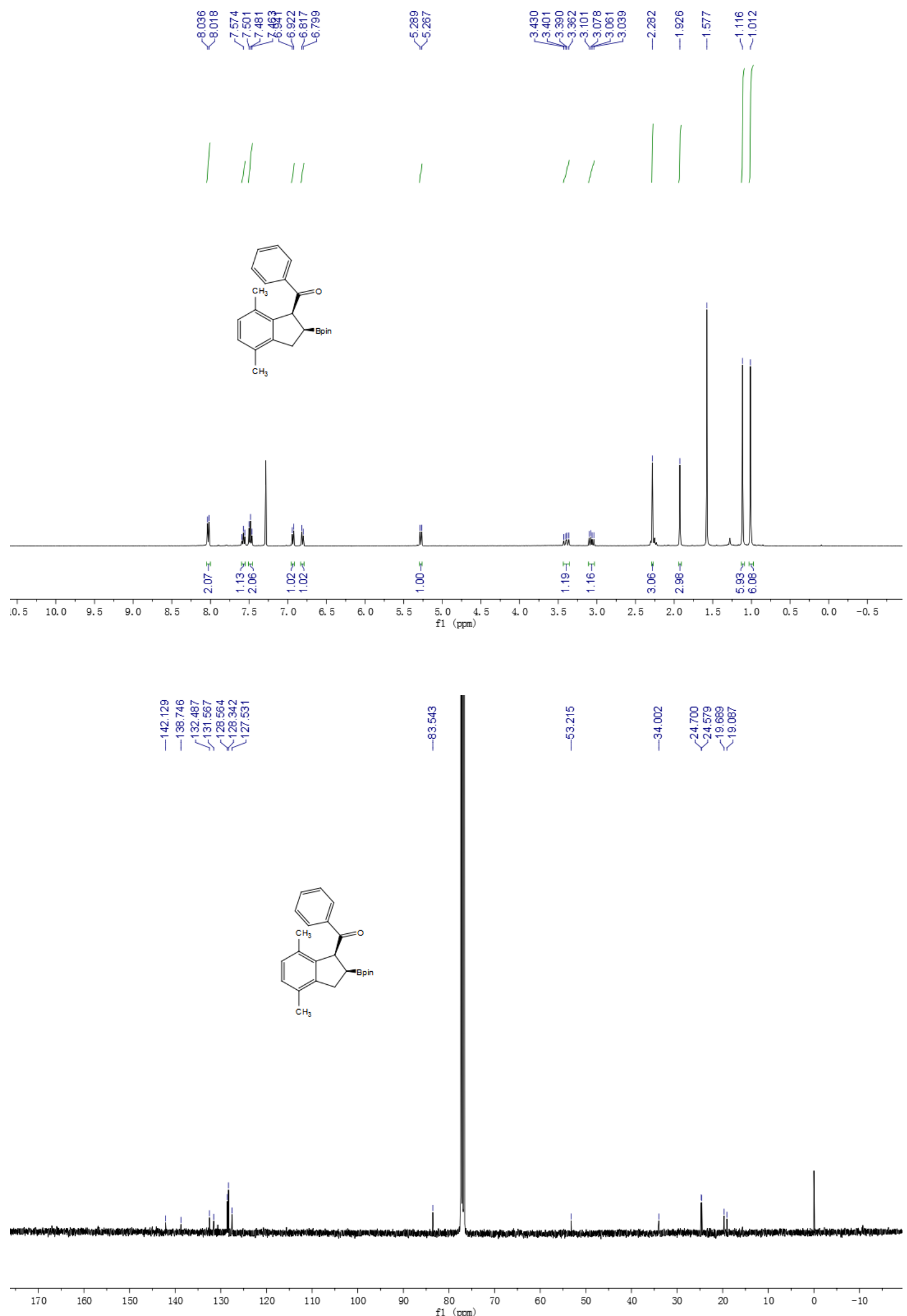
$(8 R, 9 S, 13 S, 14 S)-13-$ methyl-3-((S)-1-oxo-1-phenyl-3-(4,4,5,5-tetramethyl-1,3,2-dioxaborolan-2-yl) propan-2-yl)-6,7,8,9,11,12,13,14,15,16-decahydro-17H-cyclopenta[a]phenanthren-17-one (3w): ${ }^{1} \mathrm{H}$ NMR $400 \mathrm{MHz},{ }^{13} \mathrm{C}\{1 \mathrm{H}\}$ NMR $101 \mathrm{MHz}, \mathrm{CDCl}_{3}$

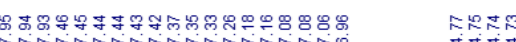

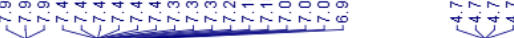

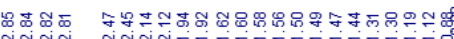

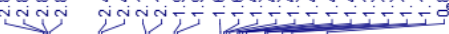
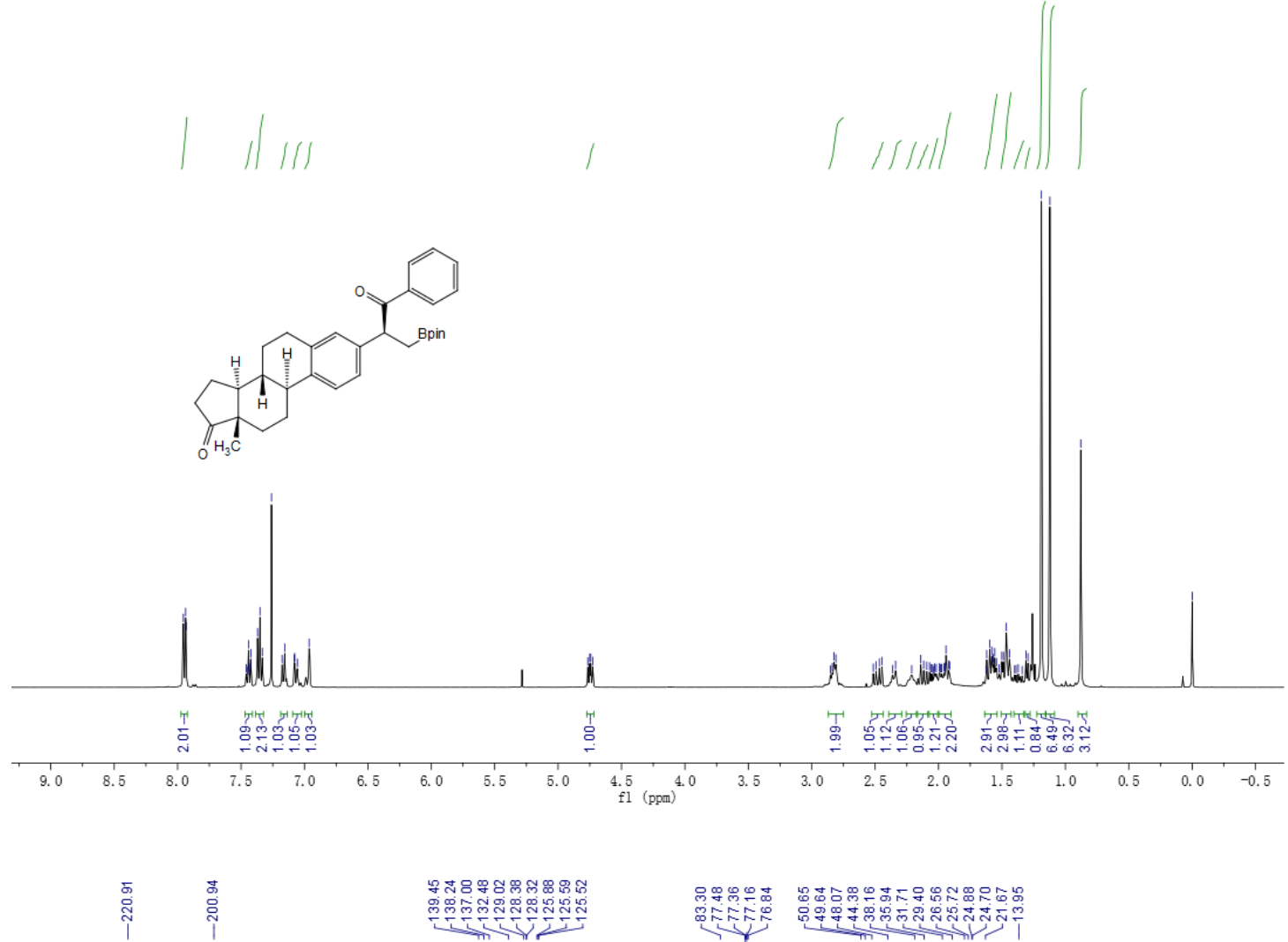

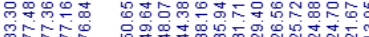

选然

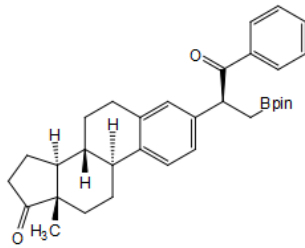

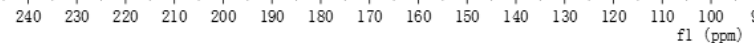


(S)-1-(4-fluorophenyl)-3-(4,4,5,5-tetramethyl-1,3,2-dioxaborolan-2-yl)-2-(p-tolyl)propan-1-one (4a): ${ }^{1} \mathrm{H}$ NMR $400 \mathrm{MHz},{ }^{13} \mathrm{C}\{1 \mathrm{H}\}$ NMR $101 \mathrm{MHz},{ }^{19} \mathrm{~F}$ NMR $376 \mathrm{MHz}, \mathrm{CDCl}_{3}$
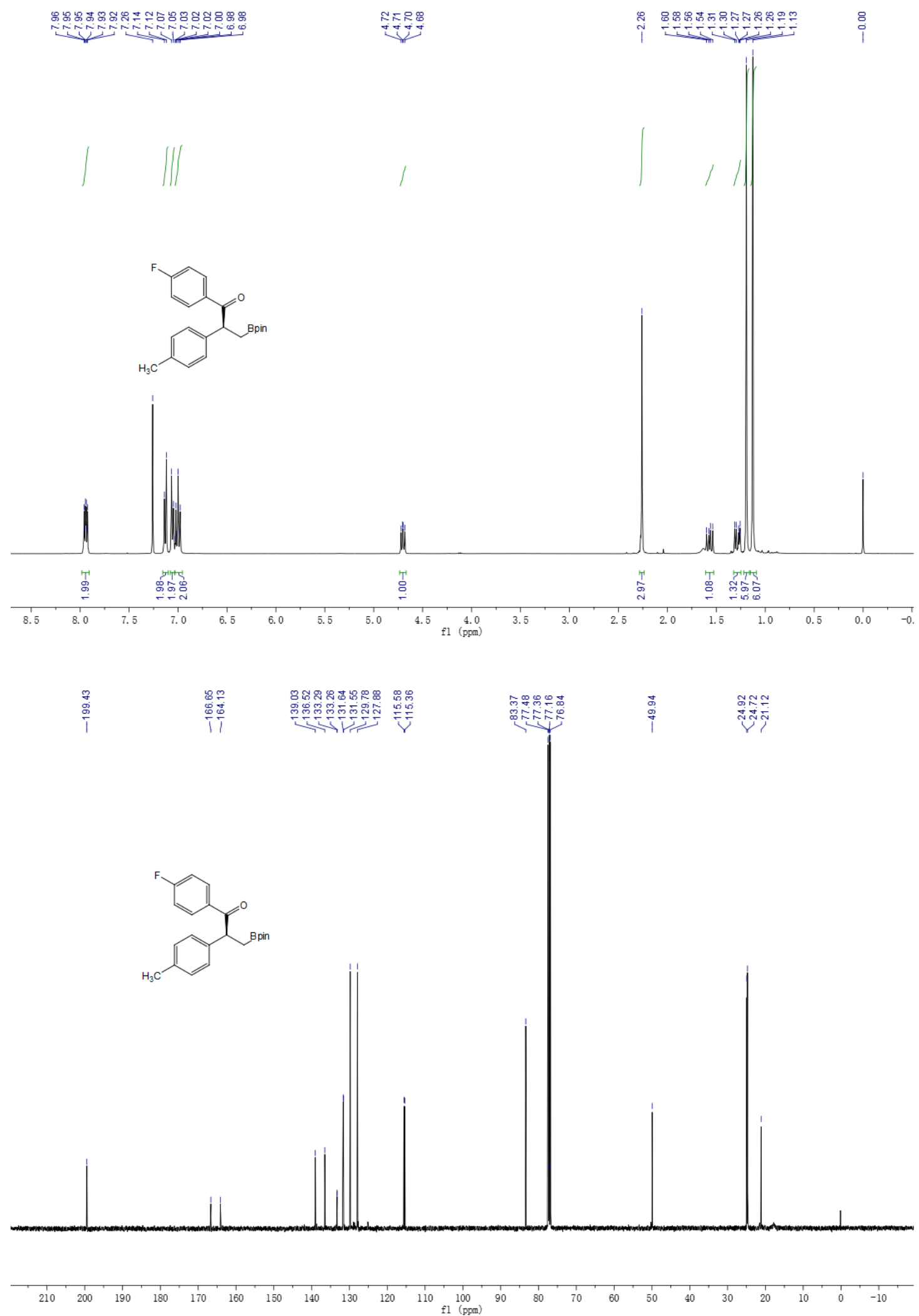


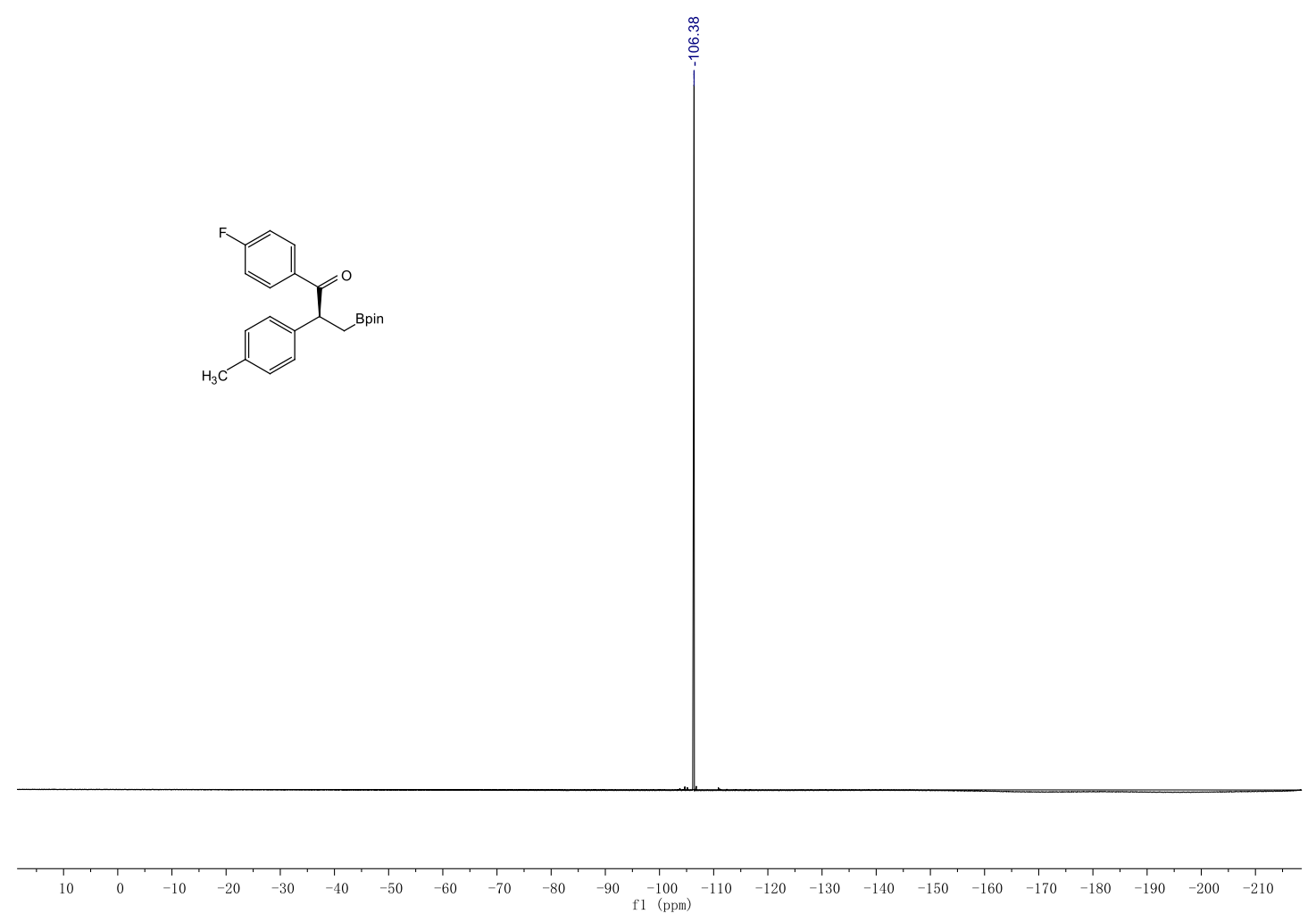


(S)-3-(4,4,5,5-tetramethyl-1,3,2-dioxaborolan-2-yl)-1,2-di-p-tolylpropan-1-one (4b): ${ }^{1} \mathrm{H}$ NMR 400 $\mathrm{MHz},{ }^{13} \mathrm{C}\{1 \mathrm{H}\}$ NMR $101 \mathrm{MHz}, \mathrm{CDCl}_{3}$

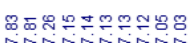

inivinitis

$\sqrt[i]{i+2}$

ขูก
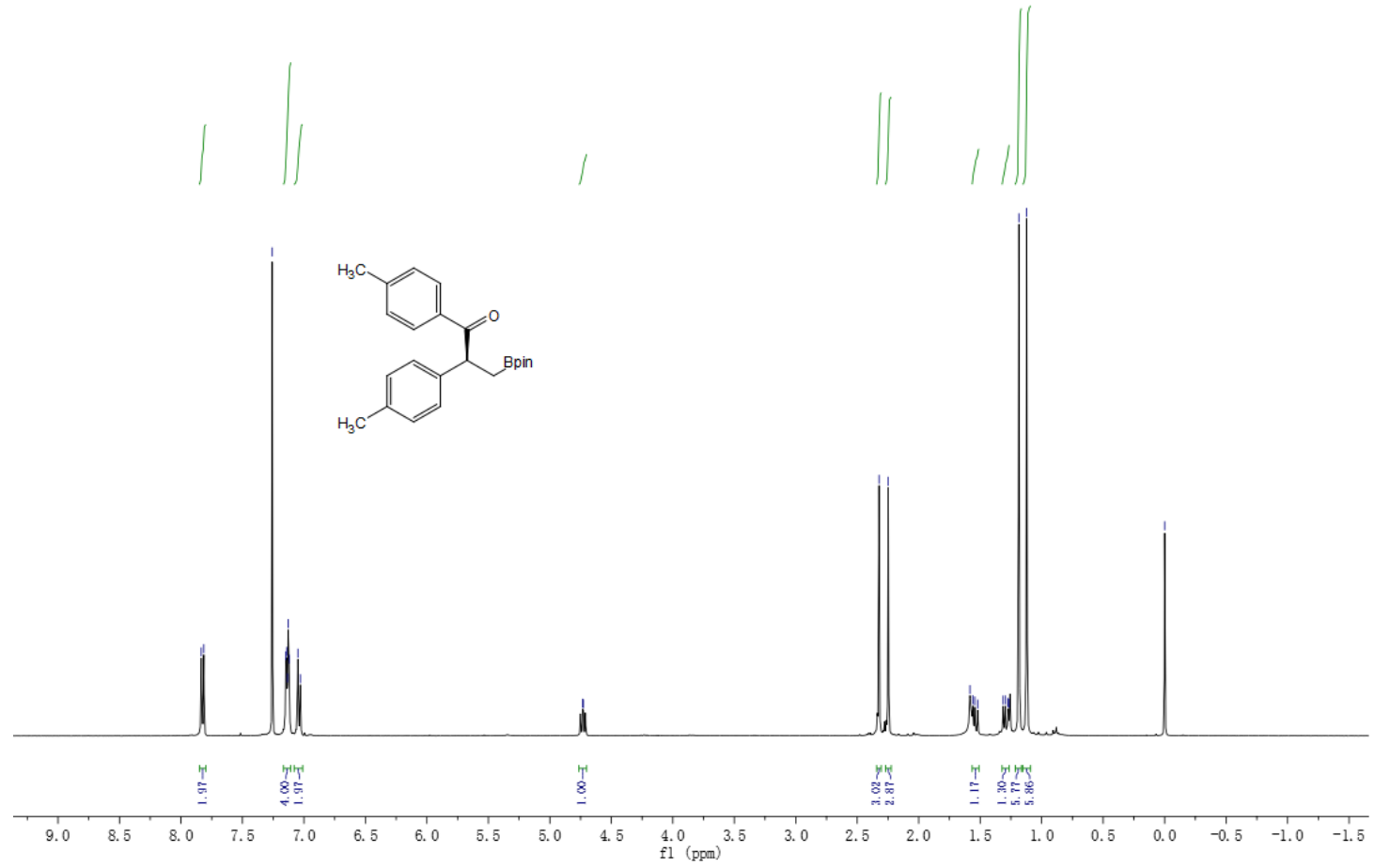

i⿱

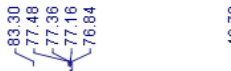

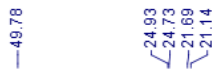<smiles>CCC(C)C(O)C1CCC(C)CC1</smiles>

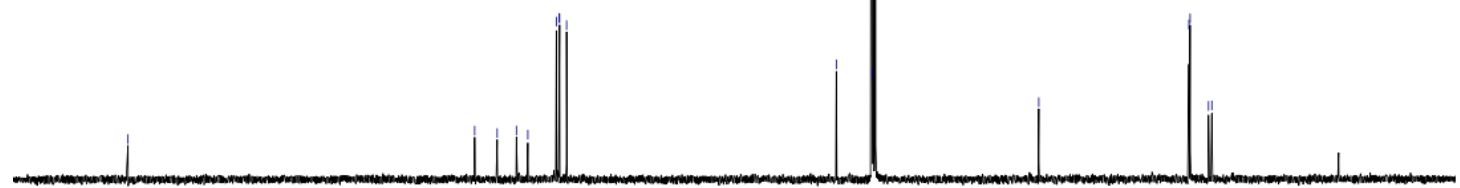

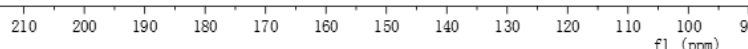


(S)-1-(4-methoxyphenyl)-3-(4,4,5,5-tetramethyl-1,3,2-dioxaborolan-2-yl)-2-(p-tolyl)propan-1-one (4c): ${ }^{1} \mathrm{H}$ NMR $400 \mathrm{MHz},{ }^{13} \mathrm{C}\{1 \mathrm{H}\} \mathrm{NMR} 101 \mathrm{MHz}, \mathrm{CDCl}_{3}$

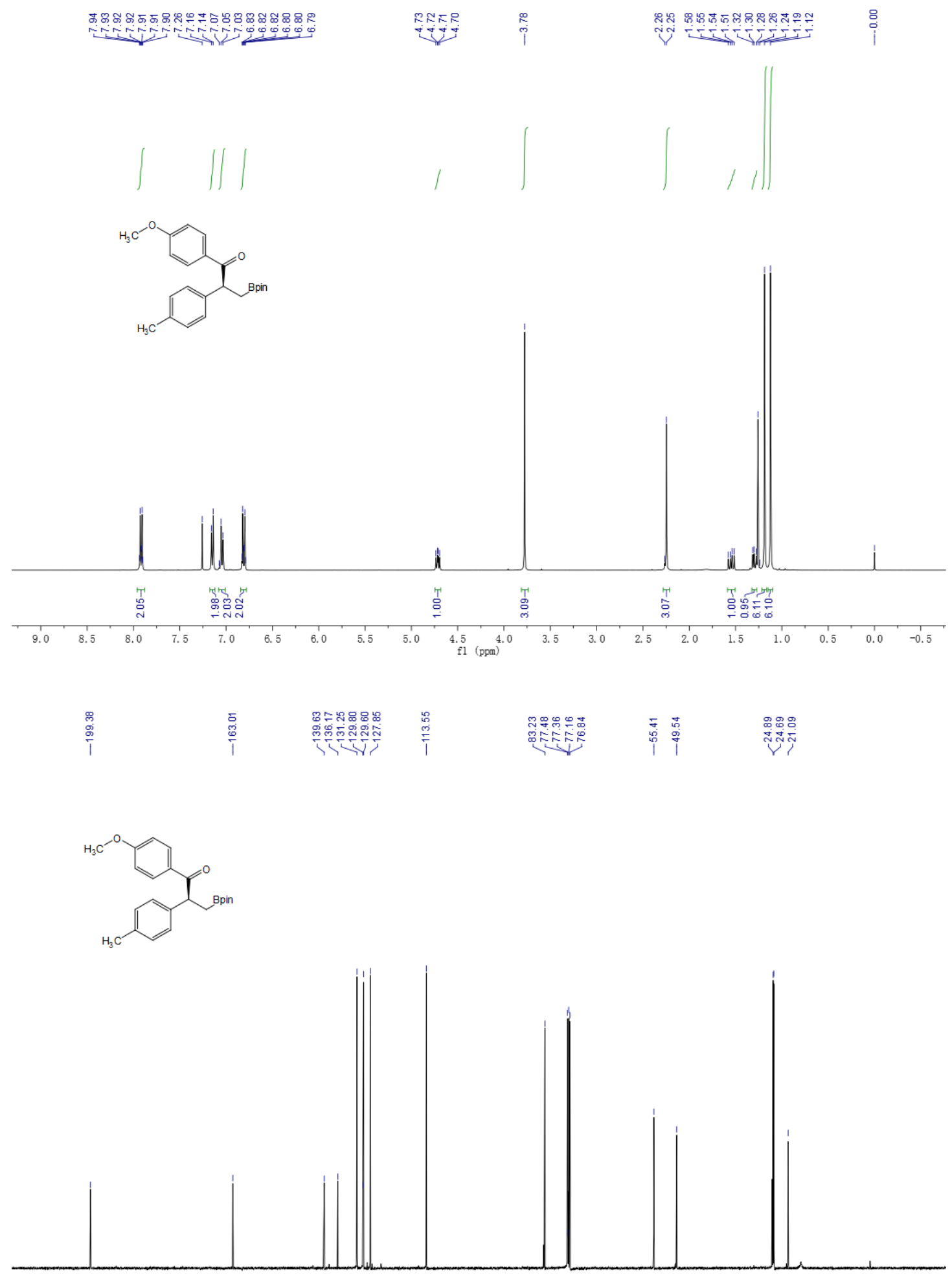

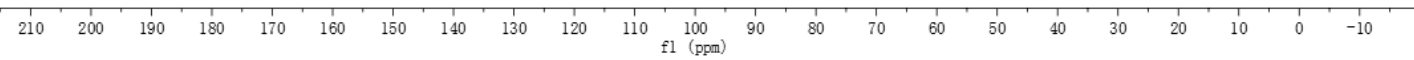


(S)-1-(4-chlorophenyl)-3-(4,4,5,5-tetramethyl-1,3,2-dioxaborolan-2-yl)-2-(p-tolyl)propan-1-one (4d): ${ }^{1} \mathrm{H}$ NMR $400 \mathrm{MHz},{ }^{13} \mathrm{C}\{1 \mathrm{H}\}$ NMR $101 \mathrm{MHz}, \mathrm{CDCl}_{3}$
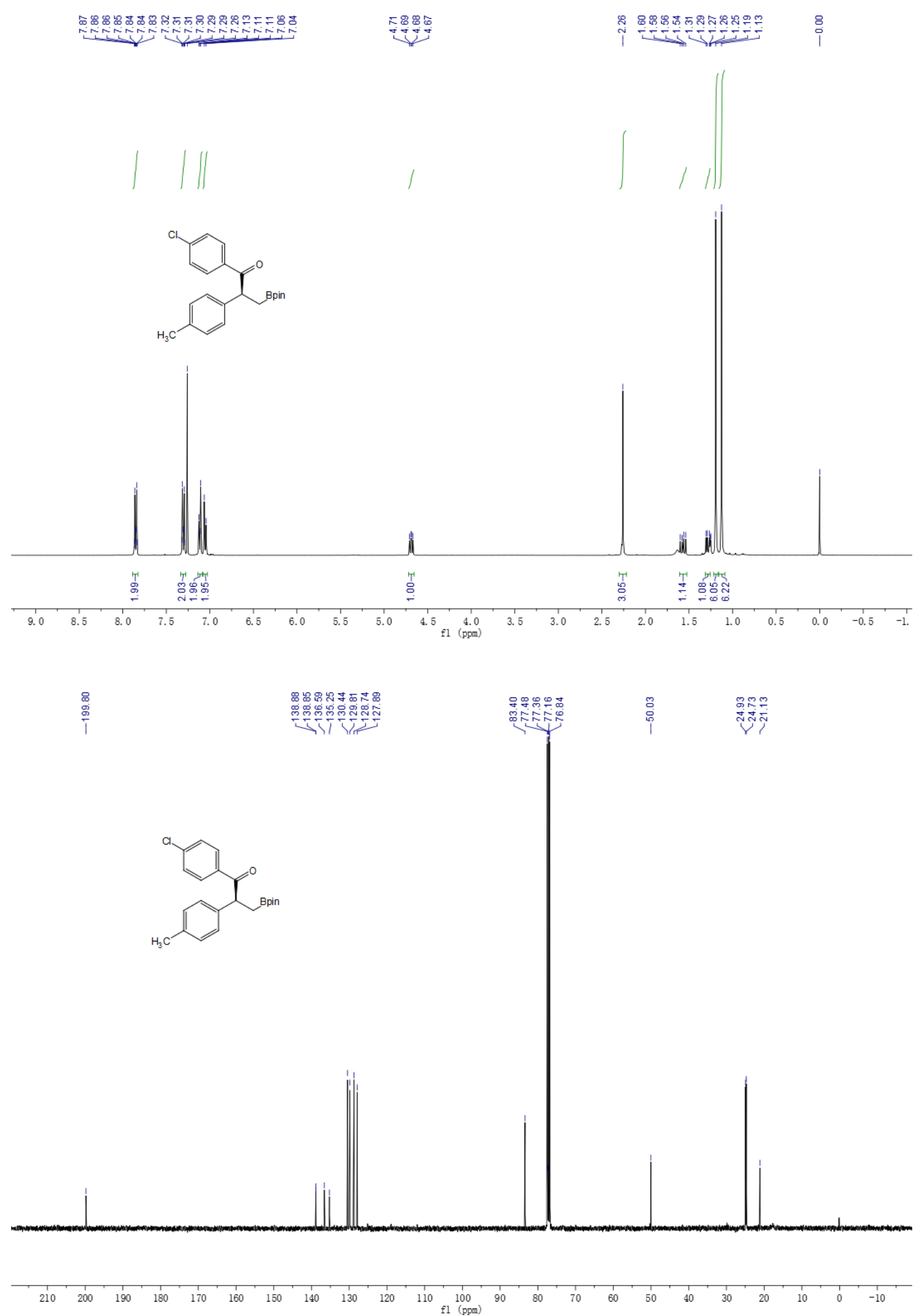
(S)-1-(furan-2-yl)-3-(4,4,5,5-tetramethyl-1,3,2-dioxaborolan-2-yl)-2-(p-tolyl)propan-1-one (4e): ${ }^{1} \mathrm{H}$ NMR $400 \mathrm{MHz},{ }^{13} \mathrm{C}\{1 \mathrm{H}\} \mathrm{NMR} 101 \mathrm{MHz}, \mathrm{CDCl}_{3}$

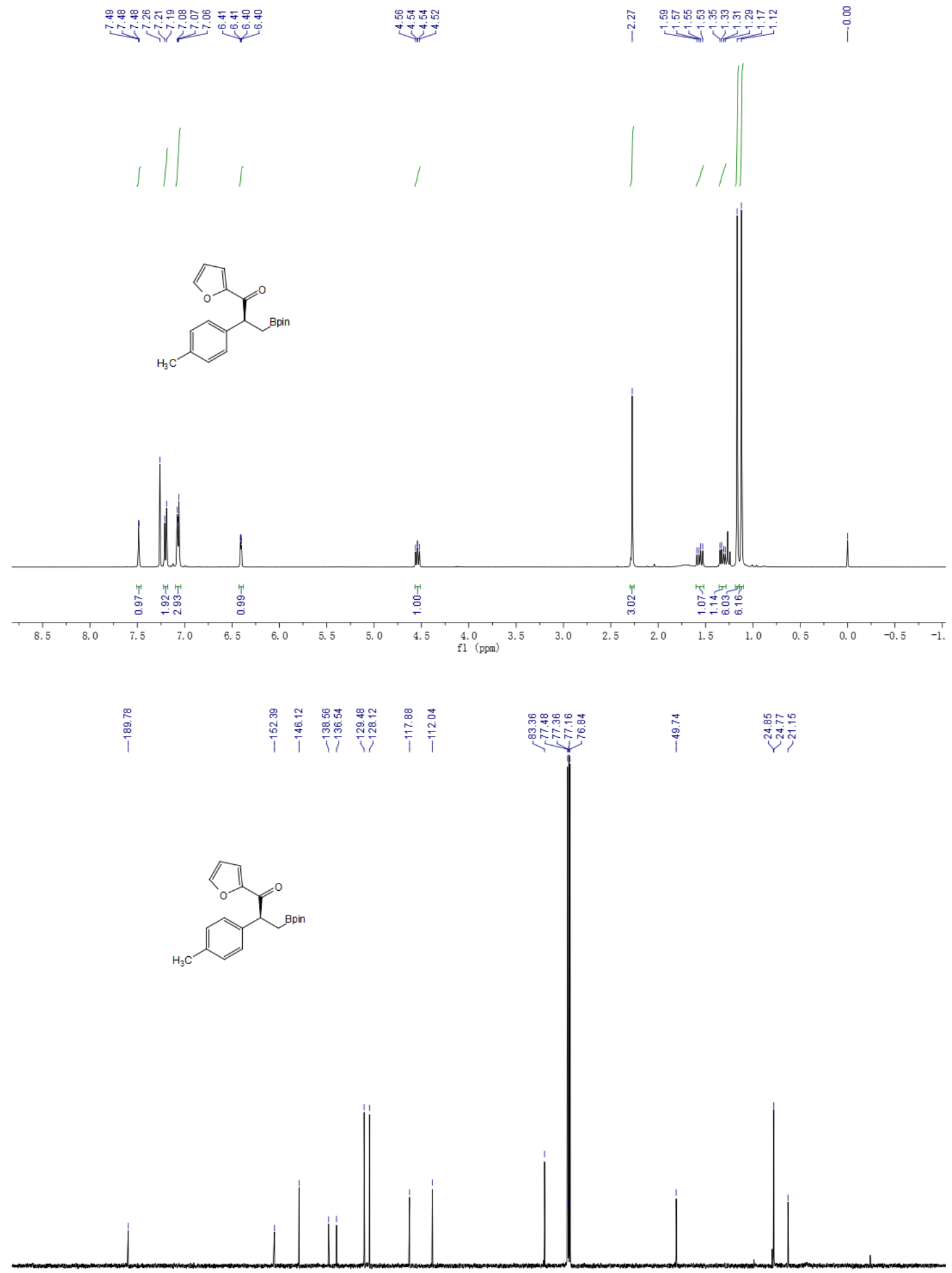

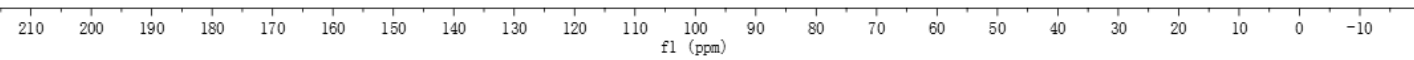


(S)-3-(4,4,5,5-tetramethyl-1,3,2-dioxaborolan-2-yl)-1-(thiophen-2-yl)-2-(p-tolyl)propan-1-one (4f): ${ }^{1} \mathrm{H}$ NMR $400 \mathrm{MHz},{ }^{13} \mathrm{C}\{1 \mathrm{H}\}$ NMR $101 \mathrm{MHz}, \mathrm{CDCl}_{3}$
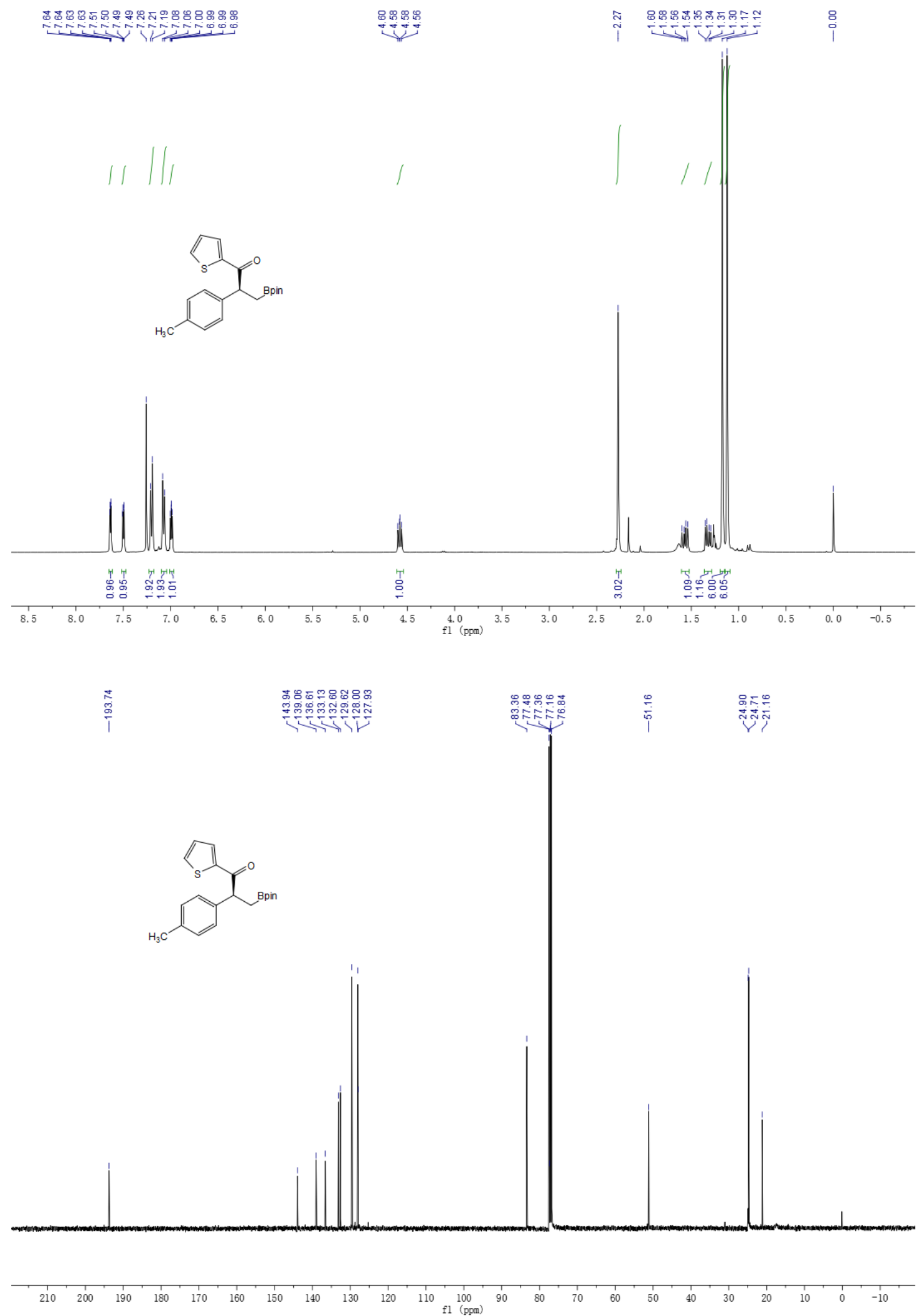
((1S,2S)-4-bromo-2-(4,4,5,5-tetramethyl-1,3,2-dioxaborolan-2-yl)-2,3-dihydro-1H-inden-1-yl)(p-to lyl)methanone (4g) $\quad{ }^{1} \mathrm{H}$ NMR $400 \mathrm{MHz},{ }^{13} \mathrm{C}\{1 \mathrm{H}\} \mathrm{NMR} 101 \mathrm{MHz}, \mathrm{CDCl}_{3}$
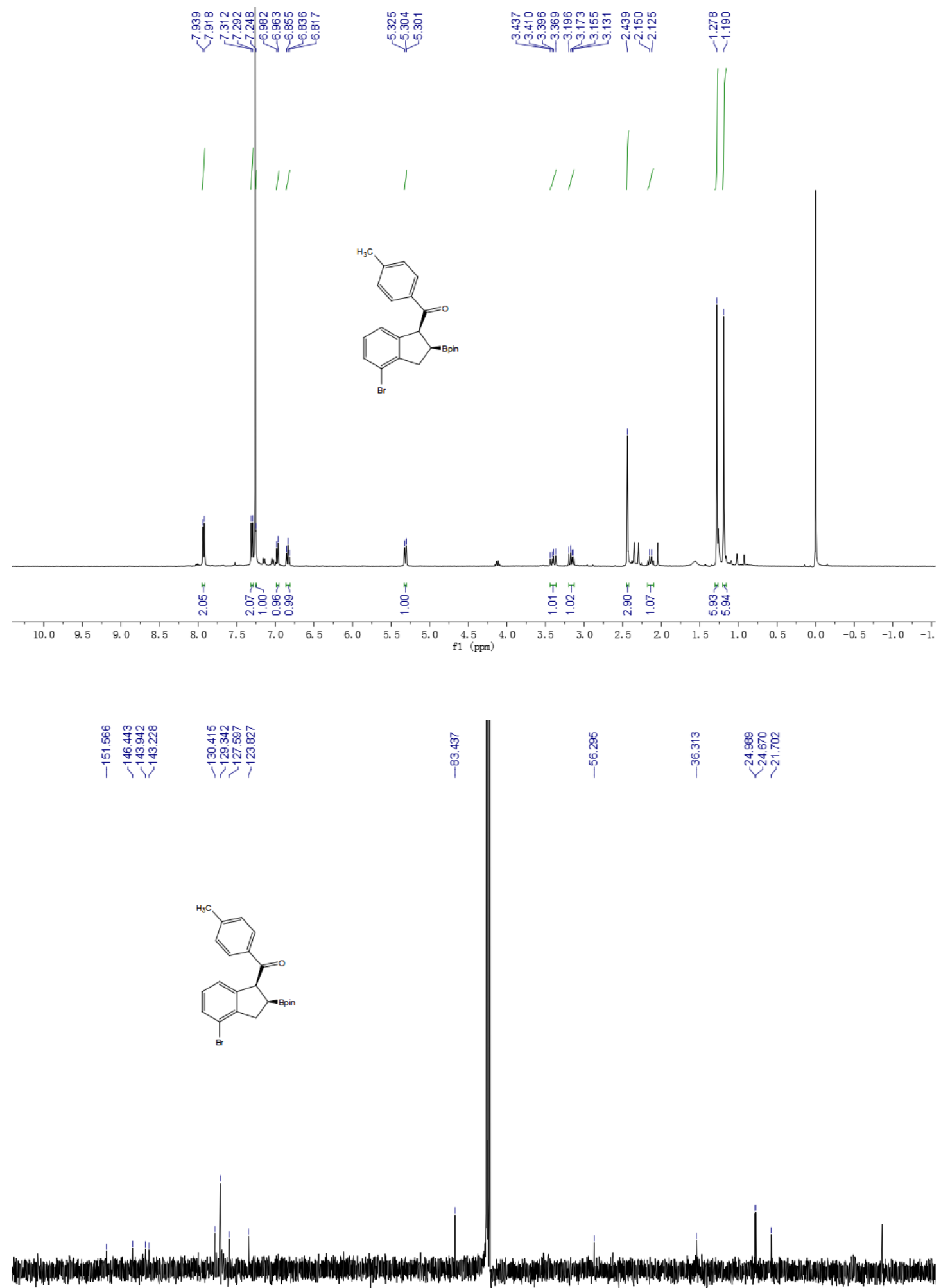

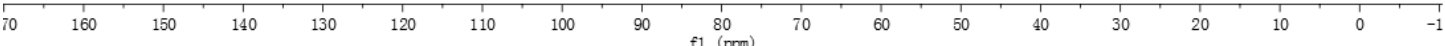


(S)-3-(3-methoxyphenyl)-1,2-diphenylpropan-1-one (5): ${ }^{1} \mathrm{H}$ NMR $400 \mathrm{MHz},{ }^{13} \mathrm{C}\{1 \mathrm{H}\}$ NMR 101 $\mathrm{MHz}, \mathrm{CDCl}_{3}$
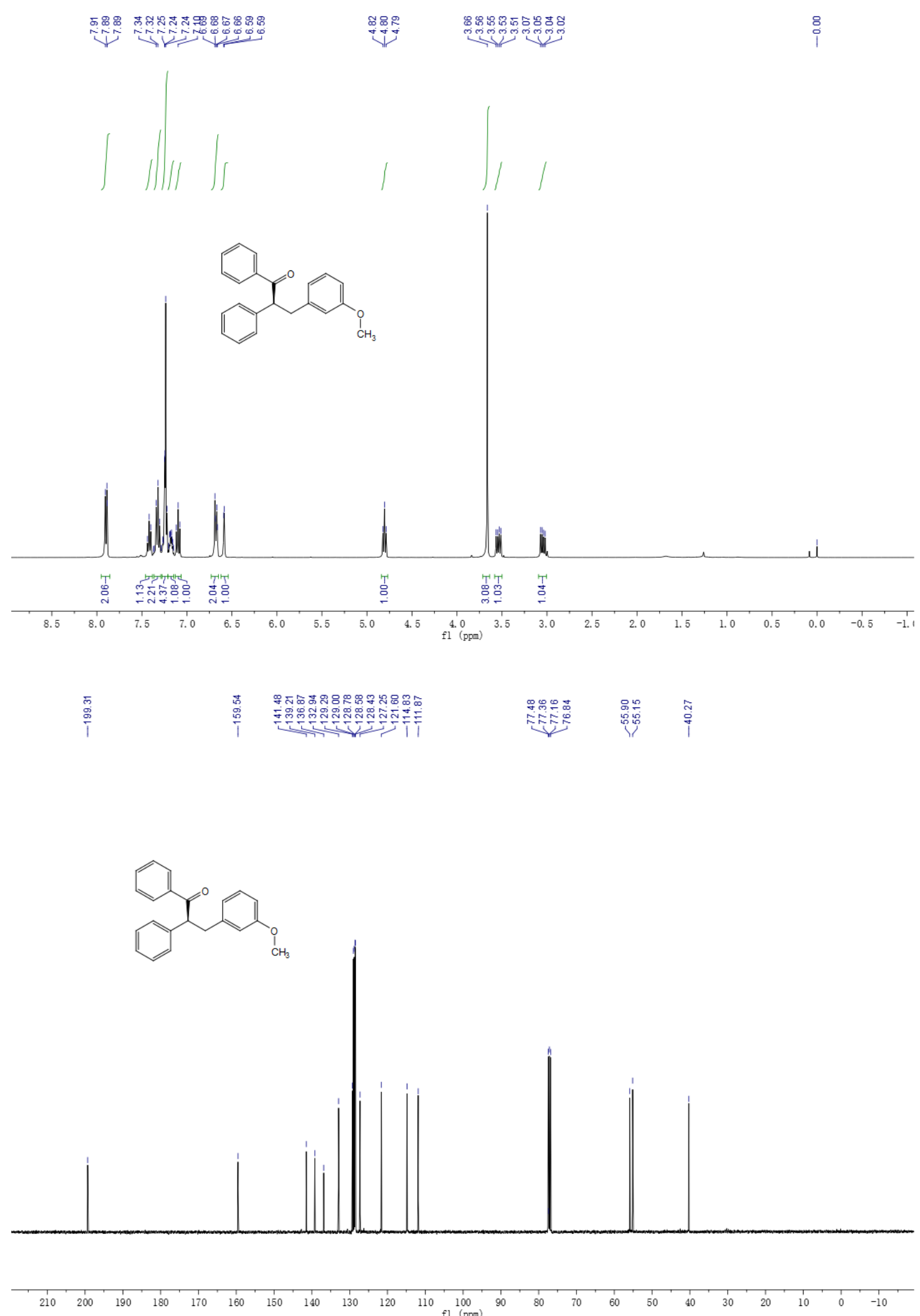
(R)-2-(4-bromophenyl)-3-hydroxy-1-phenylpropan-1-one (6): ${ }^{1} \mathrm{H}$ NMR $400 \mathrm{MHz},{ }^{13} \mathrm{C}\{1 \mathrm{H}\} \mathrm{NMR}$ $101 \mathrm{MHz}, \mathrm{CDCl}_{3}$
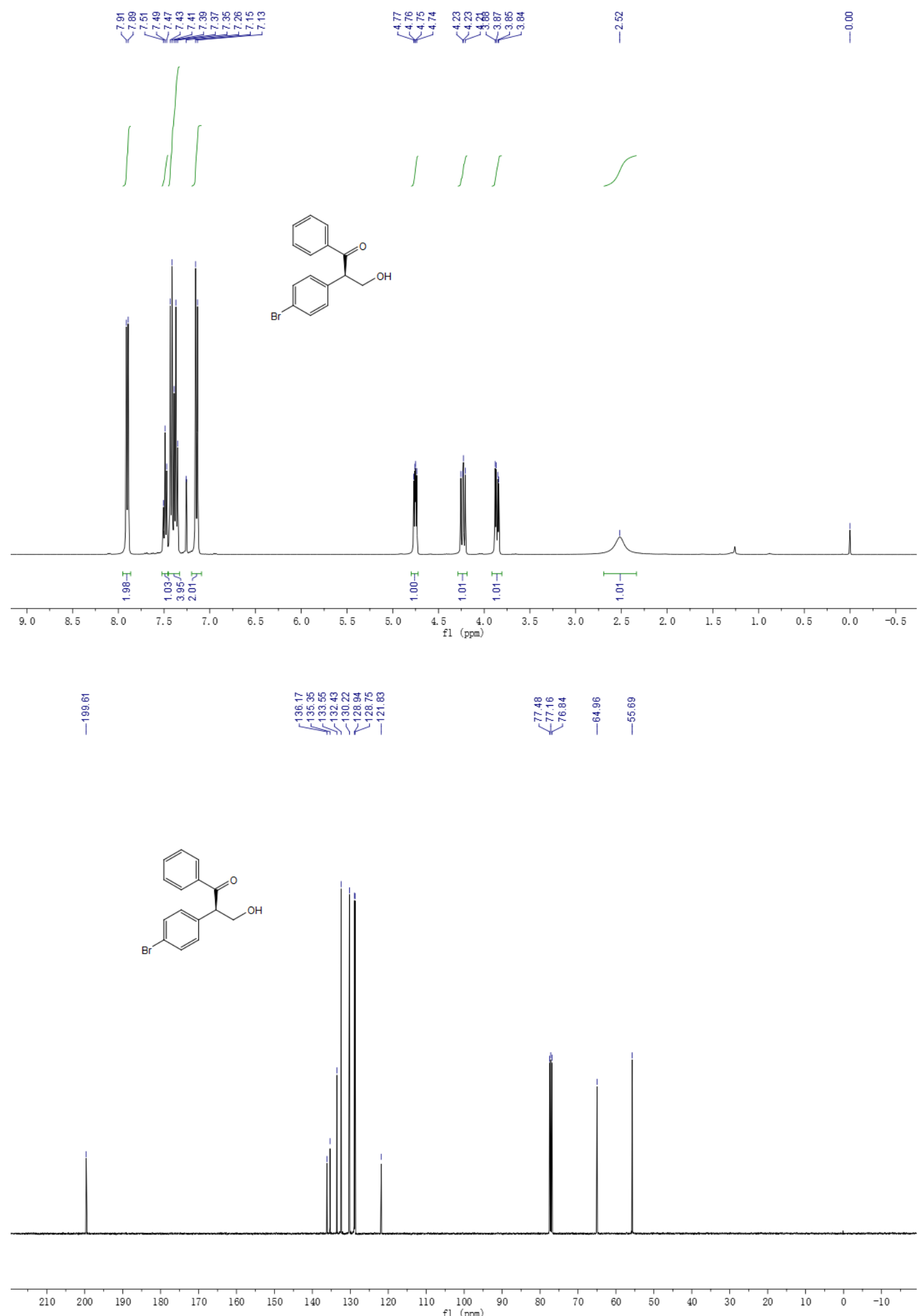